\title{
The Projection Method for Multidimensional Framelet and Wavelet Analysis
}

\author{
B. Han * \\ Department of Mathematical and Statistical Sciences, University of Alberta \\ Edmonton, Alberta T6G 2G1, Canada
}

\begin{abstract}
The projection method is a simple way of constructing functions and filters by integrating multidimensional functions and filters along parallel superplanes in the space domain. Equivalently expressed in the frequency domain, the projection method constructs a new function by simply taking a cross-section of the Fourier transform of a multidimensional function. The projection method is linked to several areas such as box splines in approximation theory and the projection-slice theorem in image processing. In this paper, we shall systematically study and discuss the projection method in the area of multidimensional framelet and wavelet analysis. We shall see that the projection method not only provides a painless way for constructing new wavelets and framelets but also is a useful analysis tool for studying various optimal properties of multidimensional refinable functions and filters. Using the projection method, we shall explicitly and easily construct a tight framelet filter bank from every box spline filter having at least order one sum rule. As we shall see in this paper, the projection method is particularly suitable to be applied to frequency-based nonhomogeneous framelets and wavelets in any dimensions, and the periodization technique is a special case of the projection method for obtaining periodic wavelets and framelets from wavelets and framelets on Euclidean spaces.
\end{abstract}

Keywords and phrases: Projection method, wavelets and framelets, tight framelets from box splines, dual framelet filter banks, interpolatory filters, orthonormal filters, frequency-based dual framelets, nonhomogeneous and homogeneous affine systems, Fourier transform

Mathematics Subject Classification: 42C40, 42C15, 41A05, 41A15

\section{Basics and Motivations on the Projection Method}

Since we frequently take a Fourier-based approach in this paper, let us recall the definition of Fourier transform first. For an integrable function $f \in L_{1}\left(\mathbb{R}^{d}\right)$, its Fourier transform is defined to be

$$
\widehat{f}(\xi):=\int_{\mathbb{R}^{d}} f(x) e^{-i 2 \pi \xi \cdot x} d x, \quad \xi \in \mathbb{R}^{d} .
$$

The above Fourier transform can be naturally extended to $L_{2}\left(\mathbb{R}^{d}\right)$ and tempered distributions through duality. Note that Fourier transform is a bijection on the space of tempered distributions on $\mathbb{R}^{d}$.

\footnotetext{
* Research supported in part by NSERC Canada under Grant RGP 228051. Corresponding author. E-mail: bhan@ualberta.ca http://www.ualberta.ca/ bhan
} 
Let $P$ be a $d \times D$ real-valued matrix such that $d \leqslant D$ and $P$ has full rank $d$. We call such a matrix $P$ a projection matrix from dimension $D$ to dimension $d$. For an integrable function $f \in L_{1}\left(\mathbb{R}^{D}\right)$, we define a projected function $P f$ on $\mathbb{R}^{d}$ by

$$
\widehat{P f}(\xi):=\widehat{f}\left(P^{\top} \xi\right), \quad \xi \in \mathbb{R}^{d} .
$$

In other words, $\widehat{P f}$ is obtained by taking a cross-section of $\widehat{f}$ on the additive subgroup $P^{\top} \mathbb{R}^{d}$ of $\mathbb{R}^{D}$. Even though the set $P^{\top} \mathbb{R}^{d}$ has Lebesgue measure zero in $\mathbb{R}^{D}$ if $d<D$, since $\widehat{f}$ is a continuous function on $\mathbb{R}^{D}$ by $f \in L_{1}\left(\mathbb{R}^{D}\right)$, the function $\widehat{P f}$ is a well-defined continuous function on $\mathbb{R}^{d}$. Moreover, in the space domain, the projected function $P f$ can be expressed as follows:

$$
[P f](x)=\frac{1}{\sqrt{\operatorname{det}\left(P P^{\top}\right)}} \int_{\left\{y \in \mathbb{R}^{D}: P y=x\right\}} f d S, \quad x \in \mathbb{R}^{d},
$$

where $S$ refers to the surface element on the superplane $\left\{y \in \mathbb{R}^{D}: P y=x\right\}$. The above relations in (1.1) and (1.2) are related to the projection-slice theorem in image processing. For the special case $d=D$, it is straightforward to deduce from (1.1) that $P f(x)=\left|\operatorname{det}\left(P^{-1}\right)\right| f\left(P^{-1} x\right)$. For a general projection matrix $P$, using singular value decomposition of $P$, we have $P=U \Sigma V^{\top}$, where $U$ is a $d \times d$ real-valued unitary matrix, $V$ is a $D \times D$ real-valued unitary matrix, and $\Sigma$ is a $d \times D$ matrix whose only nonzero entries appear in the diagonal and are denoted by $\lambda_{1}, \ldots, \lambda_{d}$ (such $\lambda_{1}, \ldots, \lambda_{d}$ are called the singular values of $P$ and must be positive since $P$ has full rank). Then $\left[V^{\top} f\right](x)=f\left(\left(V^{\top}\right)^{-1} x\right)$ and

$$
[\Sigma g]\left(x_{1}, \ldots, x_{d}\right)=\lambda_{1}^{-1} \cdots \lambda_{d}^{-1} \int_{\mathbb{R}^{D-d}} g\left(\lambda_{1}^{-1} x_{1}, \ldots, \lambda_{d}^{-1} x_{d}, x_{d+1}, \ldots, x_{D}\right) d x_{d+1} \cdots d x_{D}, \quad x_{1}, \ldots, x_{d} \in \mathbb{R} .
$$

Therefore,

$$
P f=U\left(\Sigma\left(V^{\top} f\right)\right)=\left(\Sigma\left(f\left(\left(V^{\top}\right)^{-1} \cdot\right)\right)\right)\left(U^{-1} \cdot\right) .
$$

As a consequence, since $f \in L_{1}\left(\mathbb{R}^{D}\right)$, we have $P f \in L_{1}\left(\mathbb{R}^{d}\right)$. Similarly, if $f \in L_{p}\left(\mathbb{R}^{D}\right)$ with $1 \leqslant p \leqslant \infty$ has compact support, then the above argument also shows that $P f$ is a well-defined compactly supported function in $L_{p}\left(\mathbb{R}^{d}\right)$. Let $\chi_{[0,1]^{D}}$ denote the characteristic function of the unit box $[0,1]^{D}$. Then $P \chi_{[0,1]^{D}}$ is a well-defined compactly supported function, which is a piecewise polynomial and is called a box spline in approximation theory ([1]), where $P$ is often taken to be an integer matrix. Therefore, box splines are special cases of functions obtained through the projection method by projecting the particular function $\chi_{[0,1]^{D}}$ to lower dimensions. If the projection matrix $P$ is the particular $1 \times D$ row vector $[1, \ldots, 1]$ with all entries being 1, then $P \chi_{[0,1]^{D}}$ is simply the one-dimensional B-spline function of order $D$ in approximation theory.

We now discuss projection for filters. A $d$-dimensional filter (or mask) is simply a sequence on $\mathbb{Z}^{d}$. By $l_{0}\left(\mathbb{Z}^{d}\right)$ we denote the space of all finitely supported sequences $u=\{u(k)\}_{k \in \mathbb{Z}^{d}}: \mathbb{Z}^{d} \rightarrow \mathbb{C}$. For $0<p \leqslant \infty$, by $l_{p}\left(\mathbb{Z}^{d}\right)$ we denote the space of all sequences $u$ on $\mathbb{Z}^{d}$ such that $\|u\|_{l_{p}\left(\mathbb{Z}^{d}\right)}:=\left(\sum_{k \in \mathbb{Z}^{d}}|u(k)|^{p}\right)^{1 / p}<\infty$ with the usual modification for $p=\infty$. For $u \in l_{1}\left(\mathbb{Z}^{d}\right)$, its Fourier series (or symbol) is defined to be

$$
\widehat{u}(\xi):=\sum_{k \in \mathbb{Z}^{d}} u(k) e^{-i 2 \pi k \cdot \xi}, \quad \xi \in \mathbb{R}^{d} .
$$

Since $u \in l_{1}\left(\mathbb{Z}^{d}\right), \widehat{u}$ is a $\mathbb{Z}^{d}$-periodic (i.e., $\widehat{u}(\xi+k)=\widehat{u}(\xi)$ for all $\left.k \in \mathbb{Z}^{d}\right)$ continuous function. Let $P$ be a $d \times D$ integer projection matrix. For $u \in l_{1}\left(\mathbb{Z}^{D}\right)$, we define the projected $d$-dimensional filter $P u$ by

$$
\widehat{P u}(\xi):=\widehat{u}\left(P^{\top} \xi\right), \quad \xi \in \mathbb{R}^{d} .
$$

Since $\widehat{u}$ is continuous, $\widehat{P u}$ is a well-defined continuous function. Moreover, we have $P u \in l_{1}\left(\mathbb{Z}^{d}\right)$ and

$$
[P u](m)=\sum_{k \in\left\{n \in \mathbb{Z}^{D}: P n=m\right\}} u(k), \quad m \in \mathbb{Z}^{d} .
$$


For $d=D$, we have $[P u](k)=u\left(P^{-1} k\right)$ if $k \in P \mathbb{Z}^{d}$ and $[P u](k)=0$ otherwise. That is, $P u$ is obtained from $u$ through upsampling using the square integer matrix $P$. If $u \in l_{0}\left(\mathbb{Z}^{D}\right)$ is finitely supported, then the projected filter $P u \in l_{0}\left(\mathbb{Z}^{d}\right)$ is finitely supported.

We now discuss some motivations for studying the projection method in wavelet analysis. To do so, we first recall some definitions and notation. For a $d \times d$ invertible real-valued matrix $U$ and a function $f: \mathbb{R}^{d} \rightarrow \mathbb{C}$, we shall adopt the following notation throughout the paper:

$$
f_{U ; k, n}(x):=f_{\llbracket U ; k, n \rrbracket}(x):=\llbracket U ; k, n \rrbracket f(x):=|\operatorname{det}(U)|^{1 / 2} e^{-i 2 \pi n \cdot U x} f(U x-k), \quad k, n, x \in \mathbb{R}^{d} .
$$

In particular, we define

$$
f_{U ; k}:=f_{U ; k, 0}=|\operatorname{det}(U)|^{1 / 2} f(U \cdot-k) .
$$

By duality, the definition in (1.6) can be naturally extended to a tempered distribution $f$.

Let $\mathrm{M}$ be a $d \times d$ invertible real-valued matrix. In many applications $\mathrm{M}$ is often taken to be $2 I_{d}$, where $I_{d}$ is the $d \times d$ identity matrix. Let $\Phi$ and $\Psi$ be subsets of $L_{2}\left(\mathbb{R}^{d}\right)$. A (stationary) nonhomogeneous affine system is defined to be

$$
\operatorname{AS}_{0}(\Phi ; \Psi):=\left\{\phi(\cdot-k): k \in \mathbb{Z}^{d}, \phi \in \Phi\right\} \cup\left\{\psi_{\mathrm{M}^{j} ; k}: j \in \mathbb{N}_{0}, k \in \mathbb{Z}^{d}, \psi \in \Psi\right\},
$$

where $\mathbb{N}_{0}:=\mathbb{N} \cup\{0\}$. Recall that $\{\Phi ; \Psi\}$ is a (d-dimensional) tight $\mathrm{M}$-framelet in $L_{2}\left(\mathbb{R}^{d}\right)$ if $\operatorname{AS}_{0}(\Phi ; \Psi)$ is a (normalized) tight frame for $L_{2}\left(\mathbb{R}^{d}\right)$, that is,

$$
\|f\|_{L_{2}\left(\mathbb{R}^{d}\right)}^{2}=\sum_{\phi \in \Phi} \sum_{k \in \mathbb{Z}^{d}}|\langle f, \phi(\cdot-k)\rangle|^{2}+\sum_{j=0}^{\infty} \sum_{\psi \in \Psi} \sum_{k \in \mathbb{Z}^{d}}\left|\left\langle f, \psi_{\mathrm{M}^{j} ; k}\right\rangle\right|^{2}, \quad \forall f \in L_{2}\left(\mathbb{R}^{d}\right) .
$$

If $\mathrm{AS}_{0}(\Phi ; \Psi)$ is an orthonormal basis for $L_{2}\left(\mathbb{R}^{d}\right)$, then $\{\Phi ; \Psi\}$ is called an orthonormal M-wavelet in $L_{2}\left(\mathbb{R}^{d}\right)$. Wavelets and framelets are often derived from refinable (vector) functions and (matrix) filter banks. In fact, if $\{\Phi ; \Psi\}$ is a tight $\mathrm{M}$-framelet in $L_{2}\left(\mathbb{R}^{d}\right)$ with $\Phi=\left\{\phi^{[1]}, \ldots, \phi^{[r]}\right\}$ and $\Psi=\left\{\psi^{[1]}, \ldots, \psi^{[s]}\right\}$, then it is not difficult to prove that $\Phi$ and $\Psi$ must have the following refinable structure:

$$
\widehat{\phi}\left(\mathrm{M}^{\top} \xi\right)=\widehat{a}(\xi) \widehat{\phi}(\xi) \quad \text { and } \quad \widehat{\psi}\left(\mathrm{M}^{\top} \xi\right)=\widehat{b}(\xi) \widehat{\phi}(\xi)
$$

for almost every $\xi \in \mathbb{R}^{d}$, where $\phi:=\left(\phi^{[1]}, \ldots, \phi^{[r]}\right)^{\top}, \psi:=\left(\psi^{[1]}, \ldots, \psi^{[s]}\right)^{\top}$, $\widehat{a}$ is an $r \times r$ matrix of $\mathbb{Z}^{d}$-periodic Lebesgue measurable functions, and $\widehat{b}$ is an $s \times r$ matrix of $\mathbb{Z}^{d}$-periodic Lebesgue measurable functions. The vector function $\phi$ is called an M-refinable (vector) function with a (matrix) low-pass filter $a$ and $\psi$ is an $\mathrm{M}$-wavelet vector function derived from $\phi$ with a (matrix) high-pass filter $b$. Let $P$ be an $n \times d$ integer projection matrix such that $P \mathrm{M}=\mathrm{N} P$ for some $n \times n$ invertible integer matrix $\mathrm{N}$. If (1.7) is satisfied for all $\xi \in \mathbb{R}^{d}$, then it is trivial to deduce from (1.7) that

$$
\widehat{P \phi}\left(\mathrm{N}^{\top} \zeta\right)=\widehat{P a}(\zeta) \widehat{P \phi}(\zeta) \text { and } \widehat{P \psi}\left(\mathrm{N}^{\top} \zeta\right)=\widehat{P b}(\zeta) \widehat{P \phi}(\zeta), \quad \zeta \in \mathbb{R}^{n}
$$

Hence, the projected function $P \phi$ is an N-refinable function with the projected low-pass filter $P a$ and $P \psi$ is a projected function derived from $P \phi$ with a projected high-pass filter $P b$. Therefore, the projection method has a natural connection to refinable functions in wavelet analysis. This also motivates us to study under which conditions that $\{P \Phi ; P \Psi\}$ is a tight $\mathrm{N}$-framelet in $L_{2}\left(\mathbb{R}^{n}\right)$.

There are two purposes of this paper. On one hand, results on the projection method (mainly developed by the author) in wavelet analysis are scattered through various papers in the literature and are not widely known by researchers in applied harmonic analysis. Therefore, it is appropriate for us to outline some known results and a detailed list of references on the projection method in wavelet analysis available so far in the literature. This also motivates us to further develop the projection method in wavelet analysis in this paper. On the other hand, we shall present new results on the projection method for frequency-based nonhomogeneous affine systems and frequency-based dual framelets. We shall see that 
the projection method is particularly suitable to be applied to wavelets and framelets in the frequency domain. We hope that this paper will provide the reader a self-contained introduction to and a state-ofthe-art picture on the projection method in wavelet analysis.

The structure of the paper is as follows. To motivate the reader, in Section 2 we shall first study applications of the projection method to interpolatory filters and dual framelet filter banks. Several examples will be presented to illustrate the projection method. In particular, we show that we can always easily construct a tight framelet filter bank via the projection method from every $d$-dimensional box spline filter having at least order one sum rule. In Section 3, we shall look at applications of the projection method for analyzing optimal properties of multidimensional refinable functions and subdivision schemes. In Section 4, we first recall the notion of frequency-based dual framelets from $[9,10]$. Then we study applications of the projection method to frequency-based dual framelets. In Section 5, we shall investigate multidimensional wavelets and framelets in $L_{2}\left(\mathbb{R}^{d}\right)$ by the projection method. It is well known in the literature that periodic wavelets and framelets on $\mathbb{T}^{d}$ can be easily obtained by a periodization technique from wavelets and framelets on the Euclidean space $\mathbb{R}^{d}$. At the end of this paper, we shall see that periodization technique for constructing periodic wavelets and framelets can be regarded as a particular application of the projection method and can be easily generalized using a frequency-based approach.

\section{Interpolatory Filters and Dual Framelet Filter Banks by the Projection Method}

To have some rough ideas about the projection method, in this section we shall provide some motivations for introducing the projection method and then discuss its usefulness and applications in wavelet analysis. In particular, we shall study how to construct scalar interpolatory filters and dual framelet filter banks by the projection method.

For a $d \times d$ invertible integer matrix $\mathrm{M}$, we frequently use the following notation:

$$
\mathrm{d}_{\mathrm{M}}:=|\operatorname{det}(\mathrm{M})|, \quad \Gamma_{\mathrm{M}}:=\left(\mathrm{M}[0,1)^{d}\right) \cap \mathbb{Z}^{d} \quad \text { and } \quad \Omega_{\mathrm{M}}:=\left(\left(\mathrm{M}^{\top}\right)^{-1} \mathbb{Z}^{d}\right) \cap[0,1)^{d} .
$$

In other words, $\Gamma_{\mathrm{M}}=\left\{\gamma_{1}, \ldots, \gamma_{\mathrm{d}_{\mathrm{M}}}\right\}$ denotes a complete set of representatives of the distinct cosets of the quotient group $\mathbb{Z}^{d} /\left[\mathrm{M} \mathbb{Z}^{d}\right]$, while $\Omega_{\mathrm{M}}=\left\{\omega_{1}, \ldots, \omega_{\mathrm{d}_{\mathrm{M}}}\right\}$ denotes a complete set of representatives of the distinct cosets of the quotient group $\left[\left(\mathrm{M}^{\top}\right)^{-1} \mathbb{Z}^{d}\right] / \mathbb{Z}^{d}$. Note that $\Omega_{\mathrm{M}}=\left(\mathrm{M}^{\top}\right)^{-1} \Gamma_{\mathrm{M}^{\top}}$. By $\boldsymbol{\delta}$ we denote the sequence on $\mathbb{Z}^{d}$ such that $\boldsymbol{\delta}(0)=1$ and $\boldsymbol{\delta}(k)=0$ for all $k \in \mathbb{Z}^{d} \backslash\{0\}$. Recall that for $v \in l_{0}\left(\mathbb{Z}^{d}\right)$, $\widehat{v}(\xi):=\sum_{k \in \mathbb{Z}^{d}} v(k) e^{-i 2 \pi k \cdot \xi}$. In particular, we have $\widehat{\boldsymbol{\delta}}=1$.

For an integer $j$ such that $1 \leqslant j \leqslant d$, by $\partial_{j}$ we denote the partial derivative with respect to the $j$ th coordinate of $\mathbb{R}^{d}$. Define $\mathbb{N}_{0}:=\mathbb{N} \cup\{0\}$. For $\mu=\left(\mu_{1}, \ldots, \mu_{d}\right)^{\top} \in \mathbb{N}_{0}^{d}$, we define $|\mu|:=\left|\mu_{1}\right|+\cdots+\left|\mu_{d}\right|$ and $\partial^{\mu}$ the differentiation operator $\partial_{1}^{\mu_{1}} \cdots \partial_{d}^{\mu_{d}}$. For $m \in \mathbb{N}_{0}$ and two smooth functions $\mathbf{f}$, $\mathbf{g}$, we shall use the following big $\mathcal{O}$ notation

$$
\mathbf{f}(\xi)=\mathbf{g}(\xi)+\mathcal{O}\left(\left|\xi-\xi_{0}\right|^{m}\right), \quad \xi \rightarrow \xi_{0}
$$

to mean the following relation:

$$
\partial^{\mu} \mathbf{f}\left(\xi_{0}\right)=\partial^{\mu} \mathbf{g}\left(\xi_{0}\right), \quad \forall \mu \in \mathbb{N}_{0}^{d} \text { satisfying }|\mu|<m .
$$

For a scalar filter $u \in l_{0}\left(\mathbb{Z}^{d}\right)$, we say that $u$ has order $m$ sum rules with respect to $\mathrm{M}$ if $\widehat{u}(\xi+\omega)=\mathcal{O}\left(|\xi|^{m}\right)$ as $\xi \rightarrow 0$ for all $\omega \in \Omega_{\mathrm{M}} \backslash\{0\}$, or equivalently (see [8, Theorem 3.5] and [26]),

$$
\sum_{k \in \mathbb{Z}^{d}} u(\gamma+\mathrm{M} k) \mathrm{p}(\gamma+\mathrm{M} k)=\sum_{k \in \mathbb{Z}^{d}} u(\mathrm{M} k) \mathrm{p}(\mathrm{M} k) \quad \forall \mathrm{p} \in \mathbb{P}_{m-1}, \gamma \in \Gamma_{\mathrm{M}}
$$

where $\mathbb{P}_{m-1}$ denotes the space of all $d$-variate polynomials having total degree less than $m$. If $m$ is the largest such nonnegative integer, then we define $\operatorname{sr}(u, \mathrm{M}):=m$. Similarly, we say that $u$ has order $n$ 
vanishing moments if $\widehat{u}(\xi)=\mathcal{O}\left(|\xi|^{n}\right), \xi \rightarrow 0$, that is, $\sum_{k \in \mathbb{Z}^{d}} u(k) \mathrm{p}(k)=0$ for all $\mathrm{p} \in \mathbb{P}_{n-1}$. If $n$ is the largest such nonnegative integer, then we define $\operatorname{vm}(u):=n$. We say that $u$ has order $m$ linear-phase moments with phase $\mathrm{c} \in \mathbb{R}^{d}$ if $\widehat{u}(\xi)=e^{-i 2 \pi \mathrm{c} \cdot \xi}+\mathcal{O}\left(|\xi|^{m}\right)$ as $\xi \rightarrow 0$. If $m$ is the largest such integer, then we define $\operatorname{lpm}(u):=m$. For an integer projection matrix $P$, it is trivial that $\operatorname{lpm}(u) \leqslant \operatorname{lpm}(P u)$.

The study of the projection method in wavelet analysis initially originated from the study of optimal multidimensional interpolatory filters in [20, Lemma 4.1 and Corollary 4.3]. We say that $a \in l_{0}\left(\mathbb{Z}^{d}\right)$ is $a$ (scalar) interpolatory $\mathrm{M}$-wavelet filter if

$$
a(\mathrm{M} k)=|\operatorname{det}(\mathrm{M})|^{-1} \boldsymbol{\delta}(k) \quad \text { for all } k \in \mathbb{Z}^{d},
$$

or equivalently, $\sum_{\omega \in \Omega_{M}} \widehat{a}(\xi+\omega)=1$ for all $\xi \in \mathbb{R}^{d}$. For $a, \tilde{a} \in l_{0}\left(\mathbb{Z}^{d}\right)$, we say that $(a, \tilde{a})$ is a pair of (scalar) biorthogonal $\mathrm{M}$-wavelet filters, or simply $\tilde{a}$ is a dual $\mathrm{M}$-wavelet filter of $a$, if

$$
\sum_{\omega \in \Omega_{\mathrm{M}}} \overline{\widehat{a}(\xi+\omega)} \widehat{\tilde{a}}(\xi+\omega)=1 \quad \text { for all } \xi \in \mathbb{R}^{d} .
$$

In particular, we say that $a$ is an orthonormal $\mathrm{M}$-wavelet filter if $a$ is a dual $\mathrm{M}$-wavelet filter to itself, that is,

$$
\sum_{\omega \in \Omega_{\mathrm{M}}}|\widehat{a}(\xi+\omega)|^{2}=1 \quad \text { for all } \xi \in \mathbb{R}^{d} .
$$

Define $\widehat{u}(\xi):=\overline{\widehat{a}}(\xi) \widehat{\tilde{a}}(\xi)$. Then it is trivial to observe that $(a, \tilde{a})$ is a pair of biorthogonal M-wavelet filters if and only if $u$ is an interpolatory M-wavelet filter.

Recall that the $L_{p}$ smoothness of a function $f \in L_{p}\left(\mathbb{R}^{d}\right)$ with $1 \leqslant p \leqslant \infty$ is measured by its $L_{p}$ critical smoothness exponent $\operatorname{sm}_{p}(f)$, which is defined by

$$
\operatorname{sm}_{p}(f):=\sup \left\{n+\tau:\left\|\partial^{\mu} f-\partial^{\mu} f(\cdot-t)\right\|_{L_{p}\left(\mathbb{R}^{d}\right)} \leqslant C_{f}|t|^{\tau}, \forall|\mu|=n, t \in \mathbb{R}^{d}\right\} .
$$

Let $\mathrm{M}$ be a $d \times d$ integer matrix such that $\lim _{n \rightarrow \infty} \mathrm{M}^{-n}=0$. Let $a$ be a low-pass filter in $l_{0}\left(\mathbb{Z}^{d}\right)$ such that $\widehat{a}(0)=1$. The standard M-refinable function associated with the filter/mask $a$ is denoted by $\phi^{a}$ and is defined through

$$
\widehat{\phi^{a}}(\xi):=\prod_{j=1}^{\infty} \widehat{a}\left(\left(\mathrm{M}^{\top}\right)^{-j} \xi\right), \quad \xi \in \mathbb{R}^{d} .
$$

In wavelet analysis and computer aided geometry design, it is of great interest to construct certain desired filters $a$ such that the support of the filter $a$ (or equivalently, the support of the refinable function $\phi^{a}$ ) is very short while its standard refinable function $\phi^{a}$ is as smooth as possible. Mathematically speaking, let $K$ be a given finite subset of $\mathbb{Z}^{d}$; one often takes $K=\left[m_{1}, n_{1}\right] \times \cdots \times\left[m_{d}, n_{d}\right]$ for some integers $m_{1}, \ldots, m_{d}, n_{1}, \ldots, n_{d} \in \mathbb{Z}$. Let $F_{K}$ denote all the filters $a \in l_{0}\left(\mathbb{Z}^{d}\right)$ such that each filter $a$ vanishes outside $K$ and $a$ satisfies certain prescribed property, for example, $a$ is an interpolatory $\mathrm{M}$-wavelet filter, or an orthonormal $\mathrm{M}$-wavelet filter, or a dual $\mathrm{M}$-wavelet filter of a given filter $\tilde{a}$. The key problem is to determine or estimate $\sup _{a \in F_{K}} \operatorname{sr}(a, \mathrm{M})$ and $\sup _{a \in F_{K}} \operatorname{sm}_{p}\left(\phi^{a}\right)$ for $1 \leqslant p \leqslant \infty$, where

$$
F_{K}:=\left\{a \in l_{0}\left(\mathbb{Z}^{d}\right): \operatorname{supp}(a) \subseteq K \text { and } a \text { satisfies certain given prescribed properties }\right\} .
$$

If we construct some filter $a \in F_{K}$ such that $\operatorname{sm}_{p}\left(\phi^{a}\right)=$ or $\approx \sup _{u \in F_{K}} \operatorname{sm}_{p}\left(\phi^{u}\right)$, then $a$ achieves the optimal smoothness with respect to its support. The quantities $\sup _{a \in F_{K}} \operatorname{sr}(a, \mathrm{M})$ and $\sup _{a \in F_{K}} \operatorname{sm}_{p}\left(\phi^{a}\right)$ can be analyzed in dimension one for a short support set $K$ because (1) the filters in $F_{K}$ often have few free parameters and therefore, the structure of $F_{K}$ is relatively simple, and (2) the analysis of the smoothness exponent $\operatorname{sm}_{p}\left(\phi^{a}\right)$ and $\operatorname{sr}(a, \mathrm{M})$ through the filter $a$ is relatively simple in dimension one. However, for high dimensions, quite often there are many free parameters involved in describing the set $F_{K}$ even for a very short support set $K$. It is also much more computationally expensive for estimating the smoothness exponent $\operatorname{sm}_{p}\left(\phi^{a}\right)$ for a high-dimensional (even two-dimensional) filter $a$. As a consequence, finding or 
estimating the quantity $\sup _{a \in F_{K}} \operatorname{sm}_{p}\left(\phi^{a}\right)$ is often a very challenging problem in dimensions greater than one.

In computer aided geometric design for generating smooth surfaces using subdivision schemes, it is of great importance to find $\mathscr{C}^{2}(\mathbb{R})$ interpolating $2 I_{2}$-refinable functions $\phi^{a}$ whose interpolatory filters $a$ are supported inside $[-3,3]^{2}$ and $\phi^{a}(k)=\boldsymbol{\delta}(k)$ for all $k \in \mathbb{Z}^{2}$. So, it is natural to ask whether there exists an interpolatory $2 I_{2}$-wavelet filter $a$ such that $a$ is supported inside $[-3,3]^{2}$ and $\operatorname{sm}_{\infty}\left(\phi^{a}\right)>2$. This problem has greatly motivated the author to discover the projection method in [20]. Since we require $\operatorname{sm}_{\infty}\left(\phi^{a}\right)>2$, the underlying interpolatory $2 I_{2}$-wavelet filter $a$ must have at least order 3 sum rules (see [20]). Let $K:=[-3,3]^{2}$ and $F_{K}$ be the set of all interpolatory $2 I_{2}$-wavelet filters $a$ such that each filter $a$ is supported inside $K$ and $\operatorname{sr}\left(a, 2 I_{2}\right) \geqslant 3$. By solving a system of linear equations, we can describe all elements in $F_{K}$ as a parameterized family of filters. Let $P=[1,0]$. Despite the fact that there are infinitely many filters in $F_{K}$, it has been observed through experiments by the author in 1997 that the projected filter $P a$ is the unique interpolatory 2 -wavelet filter $\frac{1}{32}\{-1,0,9,16,9,0,-1\}_{[-3,3]}$ for all $a \in F_{K}$. That is, after projection, there is a unique one-dimensional filter in $P F_{K}:=\left\{P a: a \in F_{K}\right\}$. This led to the discovery of the projection method by linking multidimensional filters and wavelets with one-dimensional filters and wavelets through projection. As proved in [20] (also see Proposition 3.1), we have $\operatorname{sm}_{p}\left(\phi^{a}\right) \leqslant \operatorname{sm}_{p}\left(\phi^{P a}\right)$ and $\operatorname{sr}\left(a, 2 I_{d}\right) \leqslant \operatorname{sr}(P a, 2)$. Therefore, we have a natural upper bound: $\sup _{a \in F_{K}} \operatorname{sm}_{p}\left(\phi^{a}\right) \leqslant \sup _{a \in F_{K}} \operatorname{sm}_{p}\left(\phi^{P a}\right)$. The quantity $\sup _{a \in F_{K}} \operatorname{sm}_{p}\left(\phi^{P a}\right)$ can often be obtained through direct analysis of one-dimensional filters $P a, a \in F_{K}$. More importantly, as we shall see by many examples in this paper, quite often $\sup _{a \in F_{K}} \operatorname{sm}_{p}\left(\phi^{a}\right)=\sup _{a \in F_{K}} \operatorname{sm}_{p}\left(\phi^{P a}\right)$. In other words, the projection method provides us an effective upper bound $\sup _{a \in F_{K}} \operatorname{sm}_{p}\left(\phi^{P a}\right)$ for the sought difficult quantity $\sup _{a \in F_{K}} \operatorname{sm}_{p}\left(\phi^{a}\right)$.

We now explain another motivation for introducing the projection method. It is often relatively easy to construct one-dimensional filters or filter banks such as one-dimensional interpolatory wavelet filters, orthonormal wavelet filters, biorthogonal wavelet filters, as well as tight or dual framelet filter banks. One simple way of constructing high-dimensional filters, wavelets and framelets is to use tensor product. That is, for $a \in l_{0}\left(\mathbb{Z}^{m}\right)$ and $b \in l_{0}\left(\mathbb{Z}^{n}\right), a \otimes b \in l_{0}\left(\mathbb{Z}^{m+n}\right)$ is a filter on $\mathbb{Z}^{m+n}$, which is defined to be $a \otimes b(j, k):=a(j) b(k), j \in \mathbb{Z}^{m}, k \in \mathbb{Z}^{n}$. Similarly, for $f \in L_{p}\left(\mathbb{R}^{m}\right)$ and $g \in L_{p}\left(\mathbb{R}^{n}\right)$, $f \otimes g \in L_{p}\left(\mathbb{R}^{m+n}\right)$ is a function on $\mathbb{R}^{m+n}$, which is defined by $f \otimes g(x, y):=f(x) g(y), x \in \mathbb{R}^{m}, y \in \mathbb{R}^{n}$. The filter $a \otimes b$ and the function $f \otimes g$ are called tensor product (or separable) filters and functions, respectively. Though separable filters and separable wavelets/framelets can be easily constructed from lower-dimensional ones, they give preference to directions along coordinate axes and therefore, may not be desirable/preferable in applications. On the other hand, it is much more difficult and challenging to construct nonseparable filters and nonseparable wavelets/framelets. Therefore, a natural question is whether it is possible to use tensor products of one-dimensional filters and wavelets/framelets to construct nonseparable filters and nonseparable wavelets/framelets in any dimensions. This problem has motivated us in [18] to initiate the applications of the projection method for constructing nonseparable filters and nonseparable wavelets/framelets in any dimensions. See Theorems 2.1 and 2.3 for details.

Multidimensional wavelets and framelets are an integrated important part of wavelet theory and there has been a continuous considerable effort in the literature to construct nonseparable wavelets and framelets. From the viewpoints of theory and application, it is of fundamental importance to understand (at least mathematically) in which sense nonseparable wavelets and framelets have advantages over tensor product ones. [17] has been greatly motivated by this problem. Using the projection method, it has been shown in [17] (see Theorem 3.12) that though most known multidimensional wavelets are nonseparable, they often essentially carry the tensor product (more precisely, projectable) structure and therefore, they cannot be better than their corresponding tensor product wavelets, in terms of smoothness exponents within a given (rectangular) support size of the wavelets.

In this section we discuss how to employ the projection method to construct nonseparable filters and filter banks. A simple observation, which allows us to use the projection method to construct new scalar interpolatory filters, lies in that for scalar filters, all the definitions of sum rule order, scalar biorthogonal/orthonormal filters, and scalar interpolatory filters only depend on the lattice $\mathrm{MZ}^{d}$ instead 
of the matrix M. In other words, if $u$ is an interpolatory (or orthonormal) M-wavelet filter and if $\mathrm{MZ}^{d}=\mathrm{NZ}^{d}$ for some integer matrix $\mathrm{N}$, then $u$ is also an interpolatory (or orthonormal) $\mathrm{N}$-wavelet filter and $\operatorname{sr}(u, \mathrm{M})=\operatorname{sr}(u, \mathrm{~N})$.

The following result has been established in $[17,18]$ for constructing new nonseparable scalar filters from known ones by a generalized projection method.

Theorem 2.1. ([18, Theorem 3.2] and [17, Lemma 2.1]) Let $\mathrm{M}$ be a $d \times d$ invertible integer matrix and $\mathrm{N}$ be a $D \times D$ invertible integer matrix with $d \leqslant D$. Let $P$ be a $d \times D$ real-valued matrix such that

$$
P N \mathbb{Z}^{D} \subseteq \mathrm{MZ}^{d} \quad \text { and } \quad\left(\gamma+\mathrm{M} \mathbb{Z}^{d}\right) \cap P \mathbb{Z}^{D} \neq \emptyset, \quad \forall \gamma \in \mathbb{Z}^{d} .
$$

Let $\left\{\eta_{1}, \ldots, \eta_{J}\right\}$ be a complete set of representatives of the distinct cosets of the quotient group $\left[P^{\top} \mathbb{Z}^{d}\right] / \mathbb{Z}^{D}$. For any filter $u \in l_{0}\left(\mathbb{Z}^{D}\right)$, define a projected filter $P u \in l_{0}\left(\mathbb{Z}^{d}\right)$ by

$$
\widehat{P u}(\xi):=\sum_{j=1}^{J} \widehat{u}\left(P^{\top} \xi+\eta_{j}\right), \quad \xi \in \mathbb{R}^{d}
$$

or equivalently $[P u](n):=J \sum_{k \in\left\{m \in \mathbb{Z}^{D}: P m=n\right\}} u(k)$ for $n \in \mathbb{Z}^{d}$. Then $\operatorname{sr}(u, \mathrm{~N}) \leqslant \operatorname{sr}(P u, \mathrm{M})$. Moreover,

(1) If $\widehat{u}(\zeta) \geqslant 0$ for all $\zeta \in \mathbb{R}^{D}$, then $\widehat{P u}(\xi) \geqslant 0$ for all $\xi \in \mathbb{R}^{d}$.

(2) If $u$ is an interpolatory $\mathrm{N}$-wavelet filter, define a finitely supported filter $v$ on $\mathbb{Z}^{d}$ by

$$
v(k):=P u(k), \quad \forall k \in \mathbb{Z}^{d} \backslash\left[\mathrm{MZ}^{d}\right] \quad \text { and } \quad v(n):=|\operatorname{det}(\mathrm{M})|^{-1} \boldsymbol{\delta}(n), \quad \forall n \in \mathrm{MZ}^{d},
$$

then $v \in l_{0}\left(\mathbb{Z}^{d}\right)$ is an interpolatory $\mathrm{M}$-wavelet filter and $\operatorname{sr}(v, \mathrm{M}) \geqslant \operatorname{sr}(u, \mathrm{~N})$. In particular, if

$$
\left\{m \in \mathbb{Z}^{D}: P m \in \mathrm{MZ}^{d}\right\} \subseteq \mathrm{N} \mathbb{Z}^{D}
$$

is satisfied, then $v=P u$, where $P u$ is defined in (2.4).

(3) If $P$ is an integer matrix satisfying (2.3) and if $(a, \tilde{a})$ is a pair of scalar biorthogonal $\mathrm{N}$-wavelet filters such that the filter a is projectable with respect to $(P, \mathrm{~N}, \mathrm{M})$, that is,

$$
\widehat{a}\left(P^{\top} \xi+\omega\right)=0 \quad \forall \xi \in \mathbb{R}^{d}, \omega \in\left[\left(\mathrm{N}^{\top}\right)^{-1} \mathbb{Z}^{D}\right] \backslash\left[P^{\top}\left(\mathrm{M}^{\top}\right)^{-1} \mathbb{Z}^{d}+\mathbb{Z}^{D}\right],
$$

then $(P a, P \tilde{a})$ is a pair of biorthogonal $\mathrm{M}$-wavelet filters.

If $P$ is an integer matrix in Theorem 2.1, then $P u$ in (2.4) agrees with the projected filter given in (1.2). The condition in (2.3) is equivalent to saying that $P: \mathbb{Z}^{D} /\left[\mathrm{N} \mathbb{Z}^{D}\right] \rightarrow \mathbb{Z}^{d} /\left[\mathrm{M}^{d}\right]$ (essentially $P: \Gamma_{\mathrm{N}} \rightarrow \Gamma_{\mathrm{M}}$ ) with $\gamma \mapsto P \gamma$ is a well-defined onto mapping. As an application of Theorem 2.1, we have the following example on constructing interpolatory $\mathrm{M}$-wavelet filters with arbitrarily high sum rule orders.

Example 2.2. ([18, Corollary 3.4]) Let $\mathrm{M}$ be a $d \times d$ invertible integer matrix. Then there exist integer matrices $E, \Sigma, F$ such that $\mathrm{M}=E \Sigma F,|\operatorname{det}(E)|=|\operatorname{det}(F)|=1$, and $\Sigma$ is a diagonal integer matrix. Let $a$ be any tensor product interpolatory $\Sigma$-wavelet filter. Take $D=d, \mathrm{~N}=\Sigma$ and $P=E$. Then all the conditions in (2.3) and (2.5) are satisfied. By Theorem 2.1, $\mathrm{Pa}$ is an interpolatory M-wavelet filter and $\operatorname{sr}(P a, M)=\operatorname{sr}(a, \Sigma)$. Since many diagonal entries of $\Sigma$ may be 1, the constructed interpolatory filters may not have symmetry. Another choice is to take $D=d, \mathrm{~N}=|\operatorname{det}(\mathrm{M})| I_{d}$ and $P=E \Sigma /|\operatorname{det}(\mathrm{M})|$. Then all the conditions in (2.3) and (2.5) are satisfied. For any (tensor product) interpolatory $\mathrm{N}$-wavelet filter $a$, we see that $P a$ is an interpolatory $\mathrm{M}$-wavelet filter and $\operatorname{sr}(P a, \mathrm{M}) \geqslant \operatorname{sr}(a, \mathrm{~N})$. Also see [14, 24] and references therein for other methods of constructing scalar interpolatory filters.

We now discuss the projection method for constructing filter banks. For completeness, we directly discuss matrix filter banks. In the following $u \in\left(l_{0}\left(\mathbb{Z}^{d}\right)\right)^{r \times s}$ simply means that $u: \mathbb{Z}^{d} \rightarrow \mathbb{C}^{r \times s}$ is a matrix-valued filter. We first recall the definition of sum rules for a matrix filter. For a (matrix) filter 
$u \in\left(l_{0}\left(\mathbb{Z}^{d}\right)\right)^{r \times r}$ with multiplicity $r$ (that is, $\widehat{u}$ is an $r \times r$ matrix of $\mathbb{Z}^{d}$-periodic trigonometric polynomials), we say that $u$ has order $m$ sum rules with respect to $\mathrm{M}([15])$ if there exists a moment matching filter $v \in\left(l_{0}\left(\mathbb{Z}^{d}\right)\right)^{1 \times r}$ with $\widehat{v}(0) \neq 0$ such that

$$
\widehat{v}\left(\mathrm{M}^{\top} \xi\right) \widehat{u}(\xi+\omega)=\boldsymbol{\delta}(\omega) \widehat{v}(\xi)+\mathcal{O}\left(|\xi|^{m}\right), \quad \xi \rightarrow 0, \quad \forall \omega \in \Omega_{\mathrm{M}} .
$$

Recall that $\operatorname{sr}(u, M)$ denotes the highest order of sum rules satisfied by $u$ in (2.7) among all possible $v \in\left(l_{0}\left(\mathbb{Z}^{d}\right)\right)^{1 \times r}$ with $\widehat{v}(0) \neq 0$. Note that $(2.7)$ depends only on $\partial^{\mu} \widehat{v}(0),|\mu|<m$. For the scalar case $r=1$, under the additional condition $\widehat{u}(0)=1$, we see that the definition which we discussed before for scalar filters agrees with the above definition in (2.7) by choosing $v \in l_{0}\left(\mathbb{Z}^{d}\right)$ satisfying $\widehat{v}(\xi)=1 / \widehat{\phi}(\xi)+\mathcal{O}\left(|\xi|^{m}\right)$ as $\xi \rightarrow 0$, where $\phi$ is the standard M-refinable function/distrubution associated with the low-pass filter $u$ and is defined through $\widehat{\phi}(\xi):=\prod_{j=1}^{\infty} \widehat{u}\left(\left(\mathrm{M}^{\top}\right)^{-j} \xi\right), \xi \in \mathbb{R}^{d}$.

Let $\Theta, a, b_{1}, \ldots, b_{s}, \tilde{a}, \tilde{b}_{1}, \ldots, \tilde{b}_{s} \in\left(l_{0}\left(\mathbb{Z}^{d}\right)\right)^{r \times r}$ be finitely supported matrix-valued sequences on $\mathbb{Z}^{d}$. Recall that $\left(\left\{a ; b_{1}, \ldots, b_{s}\right\},\left\{\tilde{a} ; \tilde{b}_{1}, \ldots, \tilde{b}_{s}\right\}\right)_{\Theta}$ is a (matrix-valued) dual $\mathrm{M}$-framelet filter bank if

$$
\overline{\widehat{a}(\xi+\omega)}^{\top} \widehat{\Theta}\left(\mathrm{M}^{\top} \xi\right) \widehat{\tilde{a}}(\xi)+\sum_{\ell=1}^{s}{\overline{\widehat{b_{\ell}}(\xi+\omega)}}^{\top} \widehat{\tilde{b}}_{\ell}(\xi)=\boldsymbol{\delta}(\omega) \widehat{\Theta}(\xi), \quad \forall \omega \in \Omega_{\mathrm{M}}
$$

for all $\xi \in \mathbb{R}^{d}$. For the special case $\Theta=I_{r} \boldsymbol{\delta}$ which is often used in wavelet analysis, we simply write $\left(\left\{a ; b_{1}, \ldots, b_{s}\right\},\left\{\tilde{a} ; \tilde{b}_{1}, \ldots, \tilde{b}_{s}\right\}\right)$ instead of $\left(\left\{a ; b_{1}, \ldots, b_{s}\right\},\left\{\tilde{a} ; \tilde{b}_{1}, \ldots, \tilde{b}_{s}\right\}\right)_{I_{r} \boldsymbol{\delta}}$. We say that $\left\{a ; b_{1}, \ldots, b_{s}\right\}_{\Theta}$ is a tight $\mathrm{M}$-framelet filter bank if $\left(\left\{a ; b_{1}, \ldots, b_{s}\right\},\left\{a ; b_{1}, \ldots, b_{s}\right\}\right)_{\Theta}$ is a dual M-framelet filter bank. When $\Theta=I_{r} \boldsymbol{\delta}$, we write $\left\{a ; b_{1}, \ldots, b_{s}\right\}$ instead of $\left\{a ; b_{1}, \ldots, b_{s}\right\}_{I_{r} \boldsymbol{\delta}}$. We say that $\left\{a ; b_{1}, \ldots, b_{s}\right\}$ is an orthonormal $\mathrm{M}$-wavelet filter bank if it is a tight $\mathrm{M}$-framelet filter bank and $s=|\operatorname{det}(\mathrm{M})|-1$.

We have the following result on projected dual framelet filter banks.

Theorem 2.3. Let $\mathrm{M}$ be a $d \times d$ invertible integer matrix and $\mathrm{N}$ be a $D \times D$ invertible integer matrix. Let $P$ be a $d \times D$ integer projection matrix such that

$$
P \mathrm{~N}=\mathrm{MP} \quad \text { and } \quad P^{\top}\left(\mathbb{Z}^{d} \backslash\left[\mathrm{M}^{\top} \mathbb{Z}^{d}\right]\right) \subseteq \mathbb{Z}^{D} \backslash\left[\mathrm{N}^{\top} \mathbb{Z}^{D}\right] .
$$

Then $\operatorname{sr}(u, \mathrm{~N}) \leqslant \operatorname{sr}(P u, \mathrm{M})$ for any matrix filter $u \in\left(l_{0}\left(\mathbb{Z}^{D}\right)\right)^{r \times r}$. If $\left(\left\{a ; b_{1}, \ldots, b_{s}\right\},\left\{\tilde{a} ; \tilde{b}_{1}, \ldots, \tilde{b}_{s}\right\}\right)_{\Theta}$ is a finitely supported (matrix) dual $\mathrm{N}$-framelet filter bank with $\Theta, a, b_{1}, \ldots, b_{s}, \tilde{a}, \tilde{b}_{1}, \ldots, \tilde{b}_{s} \in\left(l_{0}\left(\mathbb{Z}^{D}\right)\right)^{r \times r}$, then $\left(\left\{P a ; P b_{1}, \ldots, P b_{s}\right\},\left\{P \tilde{a} ; P \tilde{b}_{1}, \ldots, P \tilde{b}_{s}\right\}\right)_{P \Theta}$ is a finitely supported dual $\mathrm{M}$-framelet filter bank with $\operatorname{sr}(a, N) \leqslant \operatorname{sr}(P a, M)$ and $\operatorname{sr}(\tilde{a}, \mathrm{~N}) \leqslant \operatorname{sr}(P \tilde{a}, \mathrm{M})$. In particular, if $\left\{a ; b_{1}, \ldots, b_{s}\right\}_{\Theta}$ is a tight $\mathrm{N}$-framelet filter bank, then $\left\{P a ; P b_{1}, \ldots, P b_{s}\right\}_{P \Theta}$ is a tight $\mathrm{M}$-framelet filter bank.

Proof. Since $P N=M P$, we have $P^{\top} M^{\top}=N^{\top} P^{\top}$. Hence, $P^{\top}\left(\mathbb{Z}^{d} \backslash\left[\mathrm{M}^{\top} \mathbb{Z}^{d}\right]\right) \subseteq \mathbb{Z}^{D} \backslash\left[\mathrm{N}^{\top} \mathbb{Z}^{D}\right]$ if and only if $P^{\top} M^{\top}\left(\left[\left(M^{\top}\right)^{-1} \mathbb{Z}^{d}\right] \backslash \mathbb{Z}^{d}\right) \subseteq N^{\top}\left(\left[\left(N^{\top}\right)^{-1} \mathbb{Z}^{D}\right] \backslash \mathbb{Z}^{D}\right)$ if and only if $N^{\top} P^{\top}\left(\left[\left(\mathrm{M}^{\top}\right)^{-1} \mathbb{Z}^{d}\right] \backslash \mathbb{Z}^{d}\right) \subseteq$ $\mathrm{N}^{\top}\left(\left[\left(\mathrm{N}^{\top}\right)^{-1} \mathbb{Z}^{D}\right] \backslash \mathbb{Z}^{D}\right)$ if and only if $P^{\top}\left(\left[\left(\mathrm{M}^{\top}\right)^{-1} \mathbb{Z}^{d}\right] \backslash \mathbb{Z}^{d}\right) \subseteq\left[\left(\mathrm{N}^{\top}\right)^{-1} \mathbb{Z}^{D}\right] \backslash \mathbb{Z}^{D}$. On the other hand, since $P^{\top} \mathrm{M}^{\top}=\mathrm{N}^{\top} P^{\top}$ and $P$ is an integer matrix, we also have $P^{\top}\left[\left(\mathrm{M}^{\top}\right)^{-1} \mathbb{Z}^{d}\right]=\left(\mathrm{N}^{\top}\right)^{-1} P^{\top} \mathbb{Z}^{d} \subseteq\left(\mathrm{N}^{\top}\right)^{-1} \mathbb{Z}^{D}$. Therefore, by (2.9), for any $\omega \in \Omega_{\mathrm{M}} \backslash\{0\}$, we must have $P^{\top} \omega \in\left(\mathrm{N}^{\top}\right)^{-1} \mathbb{Z}^{D}$ but $P^{\top} \omega \notin \mathbb{Z}^{D}$.

We first prove $\operatorname{sr}(u, \mathrm{~N}) \leqslant \operatorname{sr}(P u, \mathrm{M})$. By definition of sum rules for matrix filters, that $u$ has order $m$ sum rules with respect to $\mathrm{N}$ simply means

$$
\widehat{v}\left(\mathrm{~N}^{\top} \zeta\right) \widehat{u}(\zeta+\eta)=\boldsymbol{\delta}(\eta) \widehat{v}(\zeta)+\mathcal{O}\left(|\zeta|^{m}\right), \quad \zeta \rightarrow 0, \quad \forall \eta \in \Omega_{\mathrm{N}}
$$

where $v \in\left(l_{0}\left(\mathbb{Z}^{D}\right)\right)^{1 \times r}$ with $\widehat{v}(0) \neq 0$. Since $P \mathrm{~N}=\mathrm{M} P$, we have $\mathrm{N}^{\top} P^{\top}=P^{\top} \mathrm{M}^{\top}$ and

$$
\widehat{P v}\left(\mathrm{M}^{\top} \xi\right) \widehat{P u}(\xi+\omega)=\widehat{v}\left(P^{\top} \mathrm{M}^{\top} \xi\right) \widehat{u}\left(P^{\top} \xi+P^{\top} \omega\right)=\widehat{v}\left(\mathrm{~N}^{\top} P^{\top} \xi\right) \widehat{u}\left(P^{\top} \xi+P^{\top} \omega\right) .
$$

For $\omega \in \Omega_{\mathrm{M}}$, setting $\zeta=P^{\top} \xi$ and $\eta=P^{\top} \omega$ in (2.10), since $P^{\top} \omega \neq 0$ for all $\omega \in \Omega_{\mathrm{M}} \backslash\{0\}$, we deduce that

$$
\widehat{P v}\left(\mathrm{M}^{\top} \xi\right) \widehat{P u}(\xi+\omega)=\widehat{v}\left(\mathrm{~N}^{\top} P^{\top} \xi\right) \widehat{u}\left(P^{\top} \xi+P^{\top} \omega\right)=\boldsymbol{\delta}(\omega) \widehat{P v}(\xi)+\mathcal{O}\left(|\xi|^{m}\right), \quad \xi \rightarrow 0
$$


This proves that $P u$ must have at least order $m$ sum rules with respect to $\mathrm{M}$. Hence, we proved $\operatorname{sr}(u, \mathrm{~N}) \leqslant$ $\operatorname{sr}(P u, \mathrm{M})$.

Since $\left(\left\{a ; b_{1}, \ldots, b_{s}\right\},\left\{\tilde{a} ; \tilde{b}_{1}, \ldots, \tilde{b}_{s}\right\}\right)_{\Theta}$ is a dual $\mathrm{N}$-framelet filter bank, by definition we have

$$
\overline{\widehat{a}(\zeta+\eta)}^{\top} \widehat{\Theta}\left(\mathrm{N}^{\top} \zeta\right) \widehat{\tilde{a}}(\zeta)+\sum_{\ell=1}^{s}{\overline{\widehat{b_{\ell}}}(\zeta+\eta)}^{\top} \widehat{b_{\ell}}(\zeta)=\boldsymbol{\delta}(\eta) \widehat{\Theta}(\zeta), \quad \forall \eta \in \Omega_{\mathrm{N}}, \zeta \in \mathbb{R}^{D}
$$

For $\omega \in \Omega_{\mathrm{M}}$, we now deduce that

$$
\begin{aligned}
& {\widehat{\widehat{P a}}(\xi+\omega)^{\top}}^{\mathrm{P \Theta}}\left(\mathrm{M}^{\top} \xi\right) \widehat{P \tilde{a}}(\xi)+\sum_{\ell=1}^{s}{\overline{\widehat{P b_{\ell}}}(\xi+\omega)}^{\mathrm{\top}}{\widehat{P \tilde{b}_{\ell}}}_{(\xi)} \\
& ={\overline{\widehat{a}\left(P^{\top} \xi+P^{\top} \omega\right)}}^{\top} \widehat{\Theta}\left(P^{\top} \mathrm{M}^{\top} \xi\right) \widehat{\tilde{a}}\left(P^{\top} \xi\right)+\sum_{\ell=1}^{s}{\overline{\hat{b}_{\ell}}\left(P^{\top} \xi+P^{\top} \omega\right)}^{\top} \widehat{b}_{\ell}\left(P^{\top} \xi\right) \\
& ={\overline{\widehat{a}\left(P^{\top} \xi+P^{\top} \omega\right)}}^{\top} \widehat{\Theta}\left(N^{\top} P^{\top} \xi\right) \widehat{\tilde{a}}\left(P^{\top} \xi\right)+\sum_{\ell=1}^{s}{\overline{\widehat{b}_{\ell}\left(P^{\top} \xi+P^{\top} \omega\right)}}^{\top} \widehat{\tilde{b}}_{\ell}\left(P^{\top} \xi\right) .
\end{aligned}
$$

Setting $\zeta=P^{\top} \xi$ and $\eta=P^{\top} \omega$ in $(2.11)$, since $P^{\top} \omega \neq 0$ for all $\omega \in \Omega_{\mathrm{M}} \backslash\{0\}$, we have

$$
\overline{\widehat{P a}}(\xi+\omega)^{\mathrm{T}} \widehat{P \Theta}\left(\mathrm{M}^{\top} \xi\right) \widehat{P \tilde{a}}(\xi)+\sum_{\ell=1}^{s} \widehat{\widehat{P b}}(\xi+\omega)^{\mathrm{T}} \widehat{P \tilde{b}_{\ell}}(\xi)=\boldsymbol{\delta}(\omega) \widehat{\Theta}\left(P^{\top} \xi\right)=\boldsymbol{\delta}(\omega) \widehat{P \Theta}(\xi)
$$

Therefore, we verified that $\left(\left\{P a ; P b_{1}, \ldots, P b_{s}\right\},\left\{P \tilde{a} ; P \tilde{b}_{1}, \ldots, P \tilde{b}_{s}\right\}\right)_{P \Theta}$ is a dual M-framelet filter bank. We have already proved at the beginning that $\operatorname{sr}(a, \mathrm{~N}) \leqslant \operatorname{sr}(P a, \mathrm{M})$ and $\operatorname{sr}(\tilde{a}, \mathrm{~N}) \leqslant \operatorname{sr}(P \tilde{a}, \mathrm{M})$.

As an application of Theorems 2.1 and 2.3, in the following we present several examples of scalar tight framelet filter banks. The following example constructs a family of scalar orthonormal M-wavelet filter banks with increasing orders of vanishing moments.

Example 2.4. ([18, Corollary 3.4]) Let $\mathrm{M}$ be a $d \times d$ invertible integer matrix. Then there exist integer matrices $E, \Sigma, F$ such that $\mathrm{M}=E \Sigma F,|\operatorname{det}(E)|=|\operatorname{det}(F)|=1$, and $\Sigma$ is a diagonal integer matrix. Let $\left\{a ; b_{1}, \ldots, b_{s}\right\}$ be any tensor product scalar orthonormal $\Sigma$-wavelet filter bank with $s:=|\operatorname{det}(\mathrm{M})|-1$. Take $D=d, \mathrm{~N}=\Sigma$ and $P=E$. Then the condition in (2.9) is satisfied. By Theorem 2.3 with $r=1,\left\{P a ; P b_{1}, \ldots, P b_{s}\right\}$ is a scalar orthonormal $\mathrm{M}$-wavelet filter bank. In particular, $P a$ is a scalar orthonormal M-wavelet filter and $\operatorname{sr}(P a, \mathrm{M})=\operatorname{sr}(a, \Sigma)$.

We now discuss how to construct scalar tight framelet filter banks derived from box spline filters. Let $a_{H}=\left\{\frac{1}{2}, \frac{1}{2}\right\}_{[0,1]}$ and $b_{H}=\left\{\frac{1}{2},-\frac{1}{2}\right\}_{[0,1]}$. Then $\left\{a_{H} ; b_{H}\right\}$ is the well-known Haar orthonormal 2-wavelet filter bank in dimension one ([4]).

Theorem 2.5. Let $P$ be $a d \times D$ integer matrix such that $d \leqslant D$ and $P$ has full rank $d$. Define the $D$-dimensional Haar orthonormal $2 I_{D}$-wavelet filter bank $\left\{a ; b_{1}, \ldots, b_{2^{D}-1}\right\}:=\left\{a_{H} ; b_{H}\right\} \otimes \cdots \otimes\left\{a_{H} ; b_{H}\right\}$ by taking $D$ times tensor product here. Then $a_{P}:=P a$ is the filter for the box spline function $P \chi_{[0,1]}{ }^{D}$ satisfying $\widehat{P \chi_{[0,1]} D}(2 \xi)=\widehat{a_{P}}(\xi) \widehat{P \chi_{[0,1]} D}(\xi)$ for $\xi \in \mathbb{R}^{d}$. More explicitly,

$$
\widehat{a_{P}}(\xi):=\prod_{k \in P} \frac{1+e^{-i 2 \pi k \cdot \xi}}{2} \quad \text { and } \quad \widehat{P \chi_{[0,1]}}(\xi):=\prod_{k \in P} \frac{1-e^{-i 2 \pi k \cdot \xi}}{i 2 \pi k \cdot \xi}, \quad \xi \in \mathbb{R}^{d},
$$

where $k \in P$ means that $k$ is a column vector of $P$ and $k$ goes through all the columns of $P$ once and only once. 
(i) If $P^{\top}\left(\mathbb{Z}^{d} \backslash\left[2 \mathbb{Z}^{d}\right]\right) \subseteq \mathbb{Z}^{D} \backslash\left[2 \mathbb{Z}^{D}\right]$ (or equivalently, $\operatorname{sr}\left(a_{P}, 2 I_{d}\right) \geqslant 1$ ), then $\left\{a_{P} ; P b_{1}, \ldots, P b_{2^{D}-1}\right\}$ is a finitely supported scalar tight $2 I_{d}$-framelet filter bank such that all the high-pass filters $P b_{1}, \ldots, P b_{2 D-1}$ have support no larger than the support of the low-pass filter $a_{P}$.

(ii) If $P^{\top}\left(\mathbb{Z}^{d} \backslash\left[2 \mathbb{Z}^{d}\right]\right) \nsubseteq \mathbb{Z}^{D} \backslash\left[2 \mathbb{Z}^{D}\right]$ (or equivalently, $\operatorname{sr}\left(a_{P}, 2 I_{d}\right)=0$ ), then there does not exist finitely supported filters $\Theta, u_{1}, \ldots, u_{s} \in l_{0}\left(\mathbb{Z}^{d}\right)$ with $s \in \mathbb{N}$ and $\widehat{\Theta}(0) \neq 0$ such that $\left\{a_{P} ; u_{1}, \ldots, u_{s}\right\}_{\Theta}$ is a tight $2 I_{d}$-framelet filter bank.

Proof. We first show that $P^{\top}\left(\mathbb{Z}^{d} \backslash\left[2 \mathbb{Z}^{d}\right]\right) \subseteq \mathbb{Z}^{D} \backslash\left[2 \mathbb{Z}^{D}\right]$ if and only if $\operatorname{sr}\left(a_{p}, 2 I_{d}\right) \geqslant 1$. Suppose that $P^{\top}\left(\mathbb{Z}^{d} \backslash\left[2 \mathbb{Z}^{d}\right]\right) \subseteq \mathbb{Z}^{D} \backslash\left[2 \mathbb{Z}^{D}\right]$ holds. As we discussed in the proof of Theorem 2.3, for every $\omega \in \Omega_{2 I_{d} \backslash\{0\}}$, $P^{\top} \omega \notin \mathbb{Z}^{D}$ and $P^{\top} \omega \in 2^{-1} \mathbb{Z}^{D}$. Therefore, since the Haar filter $a$ has order 1 sum rule (that is, $\operatorname{sr}\left(a, 2 I_{D}\right)=1$ and by definition $\widehat{a}(\eta)=0$ for all $\left.\eta \in \Omega_{2 I_{D}} \backslash\{0\}\right)$, we must have $\widehat{a_{P}}(\omega)=\widehat{a}\left(P^{\top} \omega\right)=0$. Hence, $\operatorname{sr}\left(a_{P}, 2 I_{d}\right) \geqslant 1$. Suppose that $P^{\top}\left(\mathbb{Z}^{d} \backslash\left[2 \mathbb{Z}^{d}\right]\right) \subseteq \mathbb{Z}^{D} \backslash\left[2 \mathbb{Z}^{D}\right]$ fails. Then there exists $\omega \in \Omega_{2 I_{d}} \backslash\{0\}$ such that $P^{\top} \omega \in \mathbb{Z}^{D}$. Hence, $\widehat{a_{P}}(\omega)=\widehat{a}\left(P^{\top} \omega\right)=\widehat{a}(0)=1$. Therefore, we must have $\operatorname{sr}\left(a_{P}, 2 I_{d}\right)=0$. This proves the equivalence between $P^{\top}\left(\mathbb{Z}^{d} \backslash\left[2 \mathbb{Z}^{d}\right]\right) \subseteq \mathbb{Z}^{D} \backslash\left[2 \mathbb{Z}^{D}\right]$ and $\operatorname{sr}\left(a_{P}, 2 I_{d}\right) \geqslant 1$ for box spline filters.

Let $\mathrm{M}=2 I_{d}$ and $\mathrm{N}=2 I_{D}$. For item (i), the matrix $P$ satisfies the condition (2.9) in Theorem 2.3. Since the $D$-dimensional Haar orthonormal $2 I_{D}$-wavelet filter bank is a special case of tight $2 I_{d}$-framelet filter banks, it now follows directly from Theorem 2.3 that $\left\{P a ; P b_{1}, \ldots, P b_{2^{D}-1}\right\}$ is a finitely supported tight $2 I_{d}$-framelet filter bank.

For item (ii), since $P^{\top}\left(\mathbb{Z}^{d} \backslash\left[2 \mathbb{Z}^{d}\right]\right) \subseteq \mathbb{Z}^{D} \backslash\left[2 \mathbb{Z}^{D}\right]$ fails, there exists $\omega \in \Omega_{2 I_{d}} \backslash\{0\}$ such that $P^{\top} \omega \in \mathbb{Z}^{D}$. Then $\widehat{a_{P}}(0)=\widehat{a_{P}}(\omega)=1$. Suppose that there exists a finitely supported tight $2 I_{d}$-framelet filter bank $\left\{a_{P} ; u_{1}, \ldots, u_{s}\right\}_{\Theta}$. By definition we have

$$
\widehat{\Theta}(2 \xi)\left|\widehat{a_{P}}(\xi)\right|^{2}+\left|\widehat{u_{1}}(\xi)\right|^{2}+\cdots+\left|\widehat{u_{s}}(\xi)\right|^{2}=\widehat{\Theta}(\xi)
$$

and

$$
\widehat{\widehat{a_{P}}}(\xi+\omega) \widehat{\Theta}(2 \xi) \widehat{a_{P}}(\xi)+\overline{\widehat{u_{1}}}(\xi+\omega) \widehat{u_{1}}(\xi)+\cdots+\overline{\widehat{u_{s}}(\xi+\omega)} \widehat{u_{s}}(\xi)=0 .
$$

Plugging $\xi=0$ into the above two identities, we must have

$$
\widehat{\Theta}(0)\left|\widehat{a_{P}}(0)\right|^{2}+\left|\widehat{u_{1}}(0)\right|^{2}+\cdots+\left|\widehat{u_{s}}(0)\right|^{2}=\widehat{\Theta}(0)
$$

and

$$
\widehat{\widehat{a_{P}}(\omega)} \widehat{\Theta}(0) \widehat{a_{P}}(0)+\overline{\widehat{u_{1}}(\omega)} \widehat{u_{1}}(0)+\cdots+\overline{\widehat{u_{s}}(\omega)} \widehat{u_{s}}(0)=0 .
$$

Since $\widehat{a_{P}}(0)=1$, we deduce from $(2.13)$ that $\widehat{u_{1}}(0)=\cdots=\widehat{u_{s}}(0)=0$. Consequently, we deduce from (2.14) that $\widehat{\widehat{a_{P}}(\omega)} \widehat{\Theta}(0) \widehat{a_{P}}(0)=0$. Since $\widehat{\widehat{a_{P}}(\omega)} \widehat{a_{P}}(0)=1$, this forces $\widehat{\Theta}(0)=0$, which is a contradiction to our assumption $\widehat{\Theta}(0) \neq 0$. This completes the proof of item (ii).

In the following we provide three examples of tight framelet filter banks which are derived from box spline filters through Theorem 2.5.

Example 2.6. Let $d=2, D=3$, and $P$ be the projection matrix

$$
P=\left[\begin{array}{lll}
1 & 0 & 1 \\
0 & 1 & 1
\end{array}\right]
$$

Then $a_{P}$ is the filter for the box spline $P \chi_{[0,1]^{3}}$ (i.e., the three-directional hat function taking value 1 at the point $(1,1))$. A tight $2 I_{2}$-framelet filter bank $\left\{a_{P} ; u_{1}, \ldots, u_{7}\right\}$ constructed by Theorem 2.5 is given 
by

$$
\begin{array}{lll}
a_{P}=\frac{1}{8}\left[\begin{array}{lll}
0 & 1 & 1 \\
1 & 2 & 1 \\
1 & 1 & 0
\end{array}\right], & u_{1}=\frac{1}{8}\left[\begin{array}{ccc}
0 & 1 & -1 \\
1 & 0 & -1 \\
1 & -1 & 0
\end{array}\right], & u_{2}=\frac{1}{8}\left[\begin{array}{ccc}
0 & -1 & -1 \\
-1 & 0 & 1 \\
1 & 1 & 0
\end{array}\right], \\
u_{3}=\frac{1}{8}\left[\begin{array}{ccc}
0 & -1 & 1 \\
-1 & 2 & -1 \\
1 & -1 & 0
\end{array}\right], & u_{4}=\frac{1}{8}\left[\begin{array}{ccc}
0 & -1 & -1 \\
1 & 0 & -1 \\
1 & 1 & 0
\end{array}\right], & u_{5}=\frac{1}{8}\left[\begin{array}{ccc}
0 & -1 & 1 \\
1 & -2 & 1 \\
1 & -1 & 0
\end{array}\right], \\
u_{6}=\frac{1}{8}\left[\begin{array}{ccc}
0 & 1 & -1 \\
-1 & -2 & -1 \\
1 & 1 & 0
\end{array}\right], & u_{7}=\frac{1}{8}\left[\begin{array}{ccc}
0 & 1 & -1 \\
-1 & 0 & 1 \\
1 & -1 & 0
\end{array}\right]
\end{array}
$$

with all filters supported inside $[0,2]^{2}$.

If $\left\{a ; b_{1}, \ldots, b_{s}\right\}_{\Theta}$ is a scalar tight $\mathrm{M}$-framelet filter bank having $s$ high-pass filters $b_{1}, \ldots, b_{s}$ such that $b_{1}=c b_{2}$ for some constant $c \in \mathbb{C}$, then it is a trivial fact that the two high-pass filters $b_{1}, b_{2}$ can be replaced by a single high-pass filter $\sqrt{1+|c|^{2}} b_{2}$, that is, $\left\{a ; \sqrt{1+|c|^{2}} b_{2}, b_{3}, \ldots, b_{s}\right\}_{\Theta}$ is also a tight $\mathrm{M}$-framelet filter bank with the total number of high-pass filters reduced by one.

Example 2.7. Let $d=1$ and $P$ be the $1 \times D$ projection matrix $P=[1, \ldots, 1]$. Then $a_{D}^{B}:=a_{P}$ is the filter for the B-spline $P \chi_{[0,1]^{D}}$ of order $D$. A tight 2 -framelet filter bank $\left\{a_{D}^{B} ; u_{1}, \ldots, u_{2^{D}-1}\right\}$ constructed by Theorem 2.5 actually has only $D$ essentially different high-pass filters (since many projected high-pass filters are multiple to each other) instead of $2^{D}-1$ high-pass filters. The resulted tight 2 -framelet filter bank $\left\{a_{D}^{B} ; v_{1}, \ldots, v_{D}\right\}$ constructed by the projection method in Theorem 2.5 is exactly the same as the B-spline tight 2-framelet filter bank constructed in [30]. More explicitly, $\widehat{a_{D}^{B}}(\xi)=2^{-D}\left(1+e^{-i 2 \pi \xi}\right)^{D}$ and the $D$ high-pass filters are given by

$$
\sqrt{\frac{D !}{\ell !(D-\ell) !}} 2^{-D}\left(1+e^{-i 2 \pi \xi}\right)^{D-\ell}\left(1-e^{-i 2 \pi \xi}\right)^{\ell}, \quad \ell=1, \ldots, D .
$$

Example 2.8. Let $P$ be the projection matrix given in (2.15). Let $a$ be a filter for the two-dimensional box spline whose direction matrix has the column vectors $(1,0)^{\top},(0,1)^{\top},(1,1)^{\top}$ with each appearing exactly $D$ times. It is known $([3,6])$ that we can construct a finitely supported tight 2 -framelet filter bank $\left\{a_{D}^{B} ; u_{1}, u_{2}\right\}$ with only two high-pass filters derived from the B-spline filter $a_{D}^{B}$ of order $D$. Then

$$
\left\{a_{D}^{B} \otimes a_{D}^{B} \otimes a_{D}^{B} ; v_{1}, \ldots, v_{26}\right\}:=\left\{a_{D}^{B} ; u_{1}, u_{2}\right\} \otimes\left\{a_{D}^{B} ; u_{1}, u_{2}\right\} \otimes\left\{a_{D}^{B} ; u_{1}, u_{2}\right\}
$$

is a three-dimensional tight $2 I_{3}$-framelet filter bank. Applying the projection method and noting that $P\left(a_{D}^{B} \otimes a_{D}^{B} \otimes a_{D}^{B}\right)=a$, we see that $\left\{a ; P v_{1}, \ldots, P v_{26}\right\}$ is a tight $2 I_{2}$-framelet filter bank derived from the box spline filter $a$ through Theorem 2.5 .

See $[2,3,5-7,16,21,27,30]$ for many other constructions of tight framelet filter banks. See Section 5 for the connections between dual/tight framelet filter banks and dual/tight framelets in $L_{2}\left(\mathbb{R}^{d}\right)$.

\section{Optimal Properties of Multidimensional Refinable Functions and Filters by the Projection Method}

In this section we shall employ the projection method to study some optimal properties of multidimensional refinable (vector) functions and (matrix) filters.

Recall that the $L_{p}$ smoothness exponent $\operatorname{sm}_{p}(f)$ of a function $f \in L_{p}\left(\mathbb{R}^{d}\right)$ is defined in (2.2). For a vector function $f=\left(f^{[1]}, \ldots, f^{[r]}\right)^{\top}$, we define $\operatorname{sm}_{p}(f):=\min _{1 \leqslant \ell \leqslant r} \operatorname{sm}_{p}\left(f^{[\ell]}\right)$. Using (1.2) and the definition of $\operatorname{sm}_{p}(f)$, the following result has been proved in [17, Lemma 2.4]. 
Proposition 3.1. ([17, Lemma 2.4]) Let $P$ be a $d \times D$ real-valued projection matrix and $f$ be a vector of compactly supported functions in $L_{p}\left(\mathbb{R}^{D}\right)$ with $1 \leqslant p \leqslant \infty$. Then $P f$ is also a vector of compactly supported functions in $L_{p}\left(\mathbb{R}^{d}\right)$ and $\operatorname{sm}_{p}(f) \leqslant \operatorname{sm}_{p}(P f)$.

We now analyze the optimal smoothness property of a filter using the projection method. We first recall the definition of a quantity $\operatorname{sm}_{p}(u, \mathrm{M})$ with $1 \leqslant p \leqslant \infty$ from [15]. Let $u \in\left(l_{0}\left(\mathbb{Z}^{d}\right)\right)^{r \times r}$ be a finitely supported matrix filter with multiplicity $r$. Let $m=\operatorname{sr}(u, \mathrm{M})$ and assume that (2.7) holds for some $v \in\left(l_{0}\left(\mathbb{Z}^{d}\right)\right)^{1 \times r}$ with $\widehat{v}(0) \neq 0$. We define the following quantity $\operatorname{sm}_{p}(u, \mathrm{M})$ as in [15] by

$$
\operatorname{sm}_{p}(u, \mathrm{M}):=-\log _{\rho(\mathrm{M})}\left[|\operatorname{det}(\mathrm{M})|^{1-1 / p} \rho(u, \mathrm{M}, p)\right],
$$

where $\rho(\mathrm{M})$ denotes the spectral radius of the matrix $\mathrm{M}$ and

$$
\rho(u, \mathrm{M}, p):=\sup \left\{\limsup _{n \rightarrow \infty}\left\|u_{n} * b\right\|_{\left(l_{p}\left(\mathbb{Z}^{d}\right)\right)^{r \times 1}}^{1 / n}: b \in \mathcal{V}_{m, v}\right\}
$$

with $\widehat{u_{n}}(\xi):=\prod_{j=1}^{n} \widehat{u}\left(\left(\mathrm{M}^{\top}\right)^{n-j} \xi\right)=\widehat{u}\left(\left(\mathrm{M}^{\top}\right)^{n-1} \xi\right) \cdots \widehat{u}\left(\mathrm{M}^{\top} \xi\right) \widehat{u}(\xi), \widehat{u_{n} * b}(\xi):=\widehat{u_{n}}(\xi) \widehat{b}(\xi)$ and

$$
\mathcal{V}_{m, v}:=\left\{b \in\left(l_{0}\left(\mathbb{Z}^{d}\right)\right)^{r \times 1}: \widehat{v}(\xi) \widehat{b}(\xi)=\mathcal{O}\left(|\xi|^{m}\right), \quad \xi \rightarrow 0\right\} .
$$

We say that $\mathrm{M}$ is isotropic if $\mathrm{M} \sim \operatorname{diag}\left(\lambda_{1}, \ldots, \lambda_{d}\right)$ with $\left|\lambda_{1}\right|=\cdots=\left|\lambda_{d}\right|$. By an expansive matrix $\mathrm{M}$, we mean that all the eigenvalues of $\mathrm{M}$ are greater than one in modulus. The quantity $\operatorname{sm}_{p}(u, \mathrm{M})$ plays a critical role in characterizing the convergence of a (vector) cascade algorithm (or subdivision scheme) in a Sobolev space and in characterizing the $L_{p}$ smoothness of a refinable (vector) function. For example, for an isotropic matrix $M$, a cascade algorithm associated with a filter $u$ and an isotropic expansive integer matrix $M$ converges in the Sobolev space $W_{p}^{m}\left(\mathbb{R}^{d}\right):=\left\{f \in L_{p}\left(\mathbb{R}^{d}\right): \partial^{\mu} f \in L_{p}\left(\mathbb{R}^{d}\right) \forall|\mu| \leqslant m\right\}$ (More precisely, the cascade sequence $\left\{\mathcal{R}^{n} f\right\}_{n=1}^{\infty}$ converges in $W_{p}^{m}\left(\mathbb{R}^{d}\right)$ for a suitable initial function $f$, where $\left.\mathcal{R} f:=|\operatorname{det}(\mathrm{M})| \sum_{k \in \mathbb{Z}^{d}} u(k) f(\mathrm{M} \cdot-k)\right)$ if and only if $\operatorname{sm}_{p}(u, \mathrm{M})>m$. Moreover, if the integer shifts of the refinable (vector) function $\phi$, associated with the filter $u$ and an expansive integer matrix $\mathrm{M}$ through $\widehat{\phi}\left(\mathrm{M}^{\top} \cdot\right)=\widehat{u} \widehat{\phi}$ with $\widehat{\phi}(0) \neq 0$, are stable in $L_{p}\left(\mathbb{R}^{d}\right)$, then $\operatorname{sm}_{p}(\phi)=\operatorname{sm}_{p}(u, \mathrm{M})$. The relation $\operatorname{sr}(u, \mathrm{M}) \geqslant \operatorname{sm}_{p}(u, \mathrm{M})$ is always true. The reader is referred to [15] and many references therein on cascade algorithms and refinable functions.

The following result links the smoothness property of a matrix filter with that of its projected filter.

Theorem 3.2. ([25, Theorem 4]) Let $\mathrm{M}$ be a $d \times d$ invertible integer matrix and $\mathrm{N}$ be a $D \times D$ invertible integer matrix. Let $P$ be a $d \times D$ integer matrix such that

$$
P \mathrm{~N}=\mathrm{MP} \quad \text { and } \quad P \mathbb{Z}^{D}=\mathbb{Z}^{d} .
$$

Let $u \in\left(l_{0}\left(\mathbb{Z}^{D}\right)\right)^{r \times r}$ be a finitely supported filter with multiplicity $r$. Then $\operatorname{sr}(u, \mathrm{~N}) \leqslant \operatorname{sr}(P u, \mathrm{M})$ and

$$
|\operatorname{det}(\mathrm{M})|^{1-1 / p} \rho(P u, \mathrm{M}, p) \leqslant|\operatorname{det}(\mathrm{N})|^{1-1 / p} \rho(u, \mathrm{~N}, p) \quad \forall 1 \leqslant p \leqslant \infty .
$$

In particular, if $\rho(\mathrm{M})=\rho(\mathrm{N})$ (that is, the spectral radii of $\mathrm{M}$ and $\mathrm{N}$ are the same), then $\operatorname{sm}_{p}(u, \mathrm{~N}) \leqslant$ $\operatorname{sm}_{p}(P u, \mathrm{M})$ for all $1 \leqslant p \leqslant \infty$.

Theorem 3.2 has been proved in [20, Lemma 4.2] for the special case $r=1$ and $\mathrm{M}=2 I_{d}$, and in [17, Theorem 2.2] for $r=1$ and a general expansive integer matrix M. As pointed out in [11, Proposition 4.2], (3.2) implies (2.9).

We now provide examples to illustrate the projection method for analyzing optimal properties of multidimensional refinable functions and filters. To do so, let us recall the definition of Hermite interpolants ([15]). Define $\Lambda_{n}:=\left\{\mu \in \mathbb{N}_{0}^{d}:|\mu|<n\right\}$ and $\# \Lambda_{n}$ the cardinality of the set $\Lambda_{n}$. We order $\Lambda_{n}$ according to $\mu=\left(\mu_{1}, \ldots, \mu_{d}\right)^{\top} \leqslant \nu=\left(\nu_{1}, \ldots, \nu_{d}\right)^{\top}$ if either $|\mu|<|\nu|$ or if $|\mu|=|\nu|$ with $\mu_{j}=\nu_{j}$ for $j=1, \ldots, t-1$ 
and $\mu_{t}<\nu_{t}$ for some $1 \leqslant t \leqslant d$. For a $d \times d$ matrix $E$, we define the $\left(\# \Lambda_{n}\right) \times\left(\# \Lambda_{n}\right)$ matrix $S\left(E, \Lambda_{n}\right)$ ([14]) by

$$
\frac{(E x)^{\mu}}{\mu !}=\sum_{\nu \in \Lambda_{n}}\left[S\left(E, \Lambda_{m}\right)\right]_{\mu, \nu} \frac{x^{\nu}}{\nu !}, \quad \mu \in \Lambda_{n} .
$$

For a column vector $\phi=\left(\phi^{[\mu]}\right)_{\mu \in \Lambda_{n}}$ of functions on $\mathbb{R}^{d}$ and $n \in \mathbb{N}$, we say that $\phi$ is an order $n$ Hermite interpolant if $\phi \in\left(\mathscr{C}^{n-1}\left(\mathbb{R}^{d}\right)\right)^{\left(\# \Lambda_{n}\right) \times 1}$ and

$$
\left[\partial^{\nu} \phi^{[\mu]}\right](k)=\boldsymbol{\delta}(\mu-\nu) \boldsymbol{\delta}(k) \quad \forall \mu, \nu \in \Lambda_{n}, k \in \mathbb{Z}^{d} .
$$

When $n=1$, we simply say that the scalar function $\phi$ is interpolating, more explicitly, $\phi$ is continuous and $\phi(k)=\boldsymbol{\delta}(k)$ for all $k \in \mathbb{Z}^{d}$.

The following result in [15, Corollary 5.2] completely characterizes M-refinable Hermite interpolants.

Theorem 3.3. ([15, Corollary 5.2]) Let $n \in \mathbb{N}$ and $\mathrm{M}$ be a $d \times d$ expansive integer matrix. If $n>1$, we further assume that $\mathrm{M}$ is isotropic. Let $\phi$ be a compactly supported $\mathrm{M}$-refinable vector function satisfying $\widehat{\phi}\left(\mathrm{M}^{\top} \xi\right)=\widehat{a}(\xi) \widehat{\phi}(\xi)$ for some matrix filter $a \in\left(l_{0}\left(\mathbb{Z}^{d}\right)\right)^{\left(\# \Lambda_{n}\right) \times\left(\# \Lambda_{n}\right)}$. Then $\phi$ is an order $n$ Hermite interpolant if and only if

(1) $\widehat{\phi^{[0]}}(0)=1$, where $\phi^{[0]}$ is the first component of the vector $\phi$.

(2) The filter $a$ is an order $n$ Hermite interpolatory $\mathrm{M}$-wavelet filter, that is,

(i) $a(0)=|\operatorname{det}(\mathrm{M})|^{-1} S\left(\mathrm{M}^{-1}, \Lambda_{n}\right)$ and $a(\mathrm{M} k)=0$ for all $k \in \mathbb{Z}^{d} \backslash\{0\}$;

(ii) the filter a has order $n$ sum rules satisfying (2.7) with respect to $\mathrm{M}$ and with a sequence $v \in$ $\left(\ell_{0}\left(\mathbb{Z}^{d}\right)\right)^{1 \times\left(\# \Lambda_{n}\right)}$ such that $\frac{(-i \partial)^{\mu}}{\mu !} \widehat{v}(0)=e_{\mu}^{\top}, \mu \in \Lambda_{n}$, where $e_{\mu}$ denotes the $\mu$-th coordinate unit vector in $\mathbb{R}^{\left(\# \Lambda_{n}\right)}$.

(3) $\operatorname{sm}_{\infty}(a, \mathrm{M})>n-1$.

Symmetry plays an important role in Hermite subdivision schemes in computer graphics and wavelet analysis. We say that $\mathscr{G}$ is a symmetry group compatible with $\mathrm{M}([14,18])$ if $\mathscr{G}$ forms a group under matrix multiplication and each element $E \in \mathscr{G}$ is an integer matrix such that $|\operatorname{det}(E)|=1$ and $\mathrm{M}^{-1} E \mathrm{M} \in \mathscr{G}$. Two commonly used symmetry groups in wavelet analysis are $D_{4}$ and $D_{6}$ :

$$
\begin{aligned}
D_{4} & :=\left\{ \pm\left[\begin{array}{ll}
1 & 0 \\
0 & 1
\end{array}\right], \pm\left[\begin{array}{cc}
1 & 0 \\
0 & -1
\end{array}\right], \pm\left[\begin{array}{ll}
0 & 1 \\
1 & 0
\end{array}\right], \pm\left[\begin{array}{cc}
0 & 1 \\
-1 & 0
\end{array}\right]\right\}, \\
D_{6} & :=\left\{ \pm\left[\begin{array}{ll}
1 & 0 \\
0 & 1
\end{array}\right], \pm\left[\begin{array}{ll}
0 & -1 \\
1 & -1
\end{array}\right], \pm\left[\begin{array}{ll}
-1 & 1 \\
-1 & 0
\end{array}\right], \pm\left[\begin{array}{ll}
0 & 1 \\
1 & 0
\end{array}\right], \pm\left[\begin{array}{ll}
1 & -1 \\
0 & -1
\end{array}\right], \pm\left[\begin{array}{ll}
-1 & 0 \\
-1 & 1
\end{array}\right]\right\} .
\end{aligned}
$$

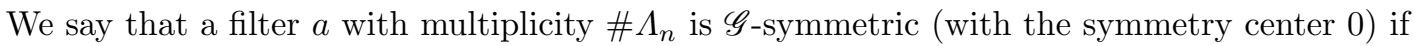

$$
a(E k)=S\left(\mathrm{M}^{-1} E \mathrm{M}, \Lambda_{n}\right) a(k) S\left(E^{-1}, \Lambda_{n}\right) \quad \forall k \in \mathbb{Z}^{d}, E \in \mathscr{G} .
$$

We now study optimal refinable Hermite interpolants using the projection method. To do so, we first recall a family of univariate Hermite interpolatory filters which have been obtained in [19, Theorem 4.3].

Proposition 3.4. ([19, Theorem 4.3]) Let $m>1$ be an integer and $n \in \mathbb{N}$. For any positive integers $\ell$ and $h$, there exists a unique order $n$ Hermite interpolatory $m$-wavelet filter $a_{m, n ; \ell, h}$ such that $a_{m, n ; \ell, h}$ is supported inside $[1-m \ell, m h-1]$ and $a_{m, n ; \ell, h}$ has order $n(\ell+h)$ sum rules with respect to the dilation factor $m$ (in fact, $\operatorname{sr}\left(a_{m, n ; \ell, h}, m\right)=n(\ell+h)$ ). Moreover, if $\ell=h$, then the order $n$ Hermite interpolatory $m$-wavelet filter $a_{m, n ; \ell, h}$ is $\{1,-1\}$-symmetric with the symmetry center 0 .

The above result in Proposition 3.4 generally fails in high dimensions by losing the uniqueness property. Nevertheless, as a generalization of [20, Lemma 4.1], we have the following result. 
Proposition 3.5. Let $m>1, \ell, h$ be positive integers. Suppose that $u$ is a scalar filter such that $u$ is supported inside $[1-m \ell, m h-1]^{d}$ and $\operatorname{sr}\left(u, m I_{d}\right) \geqslant \ell+h-1$. If $u$ has at least order $\ell+h-1$ linear-phase moments with phase 0 (for example, this condition is satisfied if $u$ is a scalar interpolatory $m I_{d}$-wavelet filter and $\left.\operatorname{sr}\left(u, m I_{d}\right) \geqslant \ell+h-1\right)$, then $[1,0, \ldots, 0] u=a_{m, 1 ; \ell, h}$.

Proof. We first show that if $u$ is a scalar interpolatory $m I_{d}$-wavelet filter and $\operatorname{sr}\left(u, m I_{d}\right) \geqslant \ell+h-1$, then $u$ has at least order $\ell+h-1$ linear-phase moments with phase 0 . Indeed, since $u$ is a scalar interpolatory $m I_{d}$-wavelet filter, by definition we have $\sum_{\omega \in \Omega_{m I}} \widehat{u}(\xi+\omega)=1$. On the other hand, by $\operatorname{sr}\left(u, m I_{d}\right) \geqslant \ell+h-1$ we must have $\widehat{u}(\xi+\omega)=\mathcal{O}\left(|\xi|^{\ell+h-1}\right)$ as $\xi \rightarrow 0$ for all $\omega \in \Omega_{m I_{d}} \backslash\{0\}$. Therefore, it is straightforward to deduce from $\sum_{\omega \in \Omega_{m I_{d}}} \widehat{u}(\xi+\omega)=1$ that $\widehat{u}(\xi)=1+\mathcal{O}\left(|\xi|^{\ell+h-1}\right)$ as $\xi \rightarrow 0$, that is, $u$ must have at least order $\ell+h-1$ linear-phase moments with phase 0 .

Since $P:=[1,0, \ldots, 0]$ satisfies the condition in (3.2) with $\mathrm{N}=m I_{d}$ and $\mathrm{M}=m$, we have $\operatorname{sr}(P u, m) \geqslant$ $\operatorname{sr}\left(u, m I_{d}\right) \geqslant \ell+h-1$. Since $u$ must have at least order $\ell+h-1$ linear-phase moments with phase 0 , we see that $P u$ also has at least order $\ell+h-1$ linear-phase moments with phase 0 . On the other hand, by a similar argument as in Proposition 3.4 and noting that $\#([1-m \ell, m h-1] \cap[m \mathbb{Z}])=\ell+h-1$, we can easily prove that $P u=a_{m, 1 ; \ell, h}$.

We now study some optimal multidimensional scalar interpolatory filters.

Example 3.6. ([20, Corollary 4.3]) Let $a$ be a $d$-dimensional scalar interpolatory $2 I_{d}$-wavelet filter (that

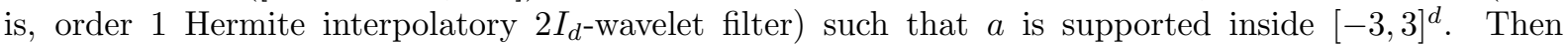
$\operatorname{sm}_{\infty}\left(a, 2 I_{d}\right) \leqslant 2$ and therefore, there is no $\mathscr{C}^{2}\left(\mathbb{R}^{d}\right)$ interpolating $2 I_{d}$-refinable function $\phi$ whose filter can be supported inside $[-3,3]^{d}$. When $a$ is $\left\{-I_{d}, I_{d}\right\}$-symmetric, we further have $[1,0, \ldots, 0] a=a_{2,1 ; 2,2}=$ $\frac{1}{32}\{-1,0,9,16,9,0,-1\}_{[-3,3]}$.

Proof. Suppose $\operatorname{sm}_{\infty}\left(a, 2 I_{d}\right)>2$. Let $P=[1,0, \ldots, 0]$ be the $1 \times d$ projection matrix. $\operatorname{Since} \operatorname{sm}_{\infty}\left(a, 2 I_{d}\right)>$ 2 , we must have $\operatorname{sr}\left(a, 2 I_{d}\right) \geqslant 3$. By Proposition 3.5, $P a$ must be an interpolatory 2 -wavelet filter. As proved in [20, Theorem 3.5], we must have $\operatorname{sm}_{\infty}(P a, 2) \leqslant \operatorname{sm}_{\infty}\left(a_{2,1 ; 2,2}, 2\right)=2$. By Theorem 3.2, we must have $\operatorname{sm}_{\infty}\left(a, 2 I_{d}\right) \leqslant \operatorname{sm}_{\infty}(P a, 2) \leqslant 2$, a contradiction. Hence, $\operatorname{sm}_{\infty}\left(a, 2 I_{d}\right) \leqslant 2$.

Using a similar idea and the projection method as in Example 3.6, we have

Example 3.7. ([23, Theorems 3.1 and 3.3]) Let $a$ be a $\left\{-I_{d}, I_{d}\right\}$-symmetric interpolatory $3 I_{d}$-wavelet filter such that $a$ is supported inside $[-5,5]^{d}$. Then $\operatorname{sm}_{\infty}\left(a, 3 I_{d}\right) \leqslant \operatorname{sm}_{\infty}\left(a_{3,1 ; 2,2}, 3\right)=\log _{3} 11 \approx 2.18266$. Moreover, if $\operatorname{sm}_{\infty}\left(a, 3 I_{d}\right)=\log _{3} 11$, then we must have

$$
[1,0, \ldots, 0] a=a_{3,1 ; 2,2}=\frac{1}{297}\{-4,-7,0,34,76,99,76,34,0,-7,-4\}_{[-5,5]} .
$$

Several optimal two-dimensional interpolatory $3 I_{2}$-wavelet filters $a$ have been reported in [23] such that each filter $a$ is supported inside $[-5,5]^{2}$, is $D_{4}$-symmetric or $D_{6}$-symmetric, and $\operatorname{sm}_{\infty}\left(a, 3 I_{2}\right)=\log _{3} 11$.

Example 3.8. ([28]) Let $a$ be a $\left\{-I_{d}, I_{d}\right\}$-symmetric interpolatory $4 I_{d}$-wavelet filter such that $a$ is supported inside $[-7,7]^{d}$. Then $\operatorname{sm}_{\infty}\left(a, 4 I_{d}\right) \leqslant \operatorname{sm}_{\infty}\left(a_{4,1 ; 2,2}, 4\right)=\log _{4} 24 \approx 2.29248$. Moreover, if $\operatorname{sm}_{\infty}\left(a, 4 I_{d}\right)=\log _{4} 24$, then we must have

$$
\begin{aligned}
{[1,0, \ldots, 0] a } & =a_{4,1 ; 2,2} \\
& =\frac{1}{768}\{-5,-12,-13,0,45,108,165,192,165,108,45,0,-13,-12,-5\}_{[-7,7]} .
\end{aligned}
$$

Several optimal two-dimensional interpolatory $4 I_{2}$-wavelet filters $a$ have been reported in [28] such that each filter $a$ is supported inside $[-7,7]^{2}$, is $D_{4}$-symmetric or $D_{6}$-symmetric, and $\operatorname{sm}_{\infty}\left(a, 4 I_{2}\right)=\log _{4} 24$. 
Example 3.9. ([12, Theorem 6] and [25, Theorems 8 and 9]) Let $a$ be a $D_{4}$-symmetric or $D_{6}$-symmetric order 2 Hermite interpolatory $2 I_{2}$-wavelet real-valued filter such that $a$ is supported inside $[-1,1]^{2}$. Then $\operatorname{sm}_{p}\left(a, 2 I_{2}\right) \leqslant 2+1 / p$ for all $1 \leqslant p \leqslant \infty$. Moreover, $\operatorname{sm}_{p}\left(a, 2 I_{2}\right)=2+1 / p$ for all $1 \leqslant p \leqslant \infty$ if $a$ is the $D_{6}$-symmetric order 2 Hermite interpolatory $2 I_{2}$-wavelet filter given by

$$
a(0,0)=\frac{1}{8}\left[\begin{array}{lll}
2 & 0 & 0 \\
0 & 1 & 0 \\
0 & 0 & 1
\end{array}\right] \quad \text { and } \quad a(1,0)=\frac{1}{32}\left[\begin{array}{ccc}
4 & -8 & 4 \\
1 & -2 & 2 \\
0 & 0 & 2
\end{array}\right]
$$

or the $D_{4}$-symmetric order 2 Hermite interpolatory $2 I_{2}$-wavelet filter given by

$$
a(0,0)=\frac{1}{8}\left[\begin{array}{lll}
2 & 0 & 0 \\
0 & 1 & 0 \\
0 & 0 & 1
\end{array}\right], \quad a(1,0)=\frac{1}{32}\left[\begin{array}{ccc}
4 & -8 & 0 \\
1 & -2 & 0 \\
0 & 0 & 2
\end{array}\right], \quad \text { and } \quad a(1,1)=\frac{1}{64}\left[\begin{array}{c}
4-8-8 \\
1-2-2 \\
1-2-2
\end{array}\right] .
$$

Therefore, there is no order $22 I_{2}$-refinable Hermite interpolant in $\mathscr{C}^{2}\left(\mathbb{R}^{2}\right)$ whose $D_{4}$ or $D_{6}$-symmetric filter can be supported inside $[-1,1]^{2}$.

Example 3.10. ([12, Theorem 7] and [25, Theorems 11 and 12]) Let $a$ be a $D_{4}$-symmetric or $D_{6^{-}}$ symmetric order 3 Hermite interpolatory $2 I_{2}$-wavelet real-valued filter such that $a$ is supported inside $[-1,1]^{2}$. Then $\operatorname{sm}_{\infty}\left(a, 2 I_{2}\right) \leqslant 3$. Therefore, there is no order $32 I_{2}$-refinable Hermite interpolant in $\mathscr{C}^{3}\left(\mathbb{R}^{2}\right)$ whose $D_{4}$ or $D_{6}$-symmetric filter can be supported inside $[-1,1]^{2}$.

We now look at applications of the projection method to multidimensional biorthogonal or orthonormal wavelets. Nonseparable multidimensional wavelets are claimed to have advantages over tensor product (separable) wavelets in numerous papers in the literature. However, as shown in [17], many nonseparable multidimensional wavelets essentially carry the tensor product structure and therefore, cannot be essentially better than tensor product wavelets. The following result is well known in the literature (e.g., see [15] and references therein).

Theorem 3.11. Let $\mathrm{M}$ be a $d \times d$ expansive integer matrix. Let $a, \tilde{a} \in\left(l_{0}\left(\mathbb{Z}^{d}\right)\right)^{r \times r}$ be finitely supported filters with multiplicity $r$ and let $\phi, \tilde{\phi}$ be compactly supported $r \times 1$ vector functions such that

$$
\widehat{\phi}\left(\mathrm{M}^{\top} \xi\right)=\widehat{a}(\xi) \widehat{\phi}(\xi), \quad \widehat{\tilde{\phi}}\left(\mathrm{M}^{\top} \xi\right)=\widehat{\tilde{a}}(\xi) \widehat{\tilde{\phi}}(\xi), \quad \text { and } \quad \overline{\widehat{\phi}}^{\top}{ }^{\mathrm{T}} \widehat{\widetilde{\tilde{\phi}}}(0)=1 .
$$

Then $(\tilde{\phi}, \phi)$ is a pair of biorthogonal functions, that is, $\tilde{\phi}, \phi \in\left(L_{2}\left(\mathbb{R}^{d}\right)\right)^{r \times 1}$ and

$$
\langle\tilde{\phi}, \phi(\cdot-k)\rangle:=\int_{\mathbb{R}^{d}} \tilde{\phi}(x) \overline{\phi(x-k)}^{\top} d x=\boldsymbol{\delta}(k) I_{r}, \quad \forall k \in \mathbb{Z}^{d},
$$

if and only if $\operatorname{sm}_{2}(a, \mathrm{M})>0, \operatorname{sm}_{2}(\tilde{a}, \mathrm{M})>0$, and $(\tilde{a}, a)$ is a pair of biorthogonal $\mathrm{M}$-wavelet filters, that is, $\sum_{\omega \in \Omega_{\mathrm{M}}} \widehat{\tilde{a}}(\xi+\omega){\overline{\widehat{a}}(\xi+\omega)^{\top}}^{\top}=I_{r}$ for all $\xi \in \mathbb{R}^{d}$.

As a direct consequence of Theorems 3.2 and 3.11, we have

Theorem 3.12. ([17, Theorem 2.5] and [11, Theorem 4.3]) Let $\mathrm{M}$ be a $d \times d$ expansive integer matrix and $\mathrm{N}$ be a $D \times D$ expansive integer matrix. Let $P$ be a $d \times D$ integer projection matrix satisfying (3.2). Let $(\tilde{\phi}, \phi)$ be a pair of biorthogonal functions such that $\widehat{\tilde{\phi}}\left(\mathrm{N}^{\top} \cdot\right)=\widehat{\tilde{a}} \widehat{\tilde{\phi}}, \widehat{\phi}\left(\mathrm{N}^{\top} \cdot\right)=\widehat{a} \widehat{\phi}$ with $\tilde{a}, a \in\left(l_{0}\left(\mathbb{Z}^{d}\right)\right)^{r \times r}$, and $\overline{\widehat{\phi}}(0)^{\top} \widehat{\tilde{\phi}}(0)=1$. If the filter a is projectable with respect to $(P, \mathrm{~N}, \mathrm{M})$ (see (2.6)), then $(P \tilde{\phi}, P \phi)$ is a pair of biorthogonal functions such that $\widehat{P \tilde{\phi}}\left(\mathrm{M}^{\top} \cdot\right)=\widehat{P \tilde{a}} \widehat{P \tilde{\phi}}$ and $\widehat{P \phi}\left(\mathrm{M}^{\top} \cdot\right)=\widehat{P a} \widehat{P \phi}$. Moreover, $\operatorname{sr}(a, \mathrm{~N}) \leqslant$ $\operatorname{sr}(P a, \mathrm{M}), \operatorname{sr}(\tilde{a}, \mathrm{~N}) \leqslant \operatorname{sr}(P \tilde{a}, \mathrm{M}), \operatorname{sm}_{p}(\phi) \leqslant \operatorname{sm}_{p}(P \phi)$, and $\operatorname{sm}_{p}(\tilde{\phi}) \leqslant \operatorname{sm}_{p}(P \tilde{\phi})$ for all $1 \leqslant p \leqslant \infty$. If in addition $\rho(\mathrm{M})=\rho(\mathrm{N})$, then we further have $\operatorname{sm}_{p}(a, \mathrm{~N}) \leqslant \operatorname{sm}_{p}(P a, \mathrm{M})$ and $\operatorname{sm}_{p}(\tilde{a}, \mathrm{~N}) \leqslant \operatorname{sm}_{p}(P \tilde{a}, \mathrm{M})$ for all $1 \leqslant p \leqslant \infty$. 
As pointed out in [17, Theorem 3.2], many multidimensional filters are projectable. For example, most box spline filters are projectable with respect to $\left([1,0, \ldots, 0], 2 I_{d}, 2\right)$. In the following, we present two examples to illustrate applications of the projection method to multidimensional wavelets.

Example 3.13. Let $a \in l_{0}\left(\mathbb{Z}^{d}\right)$ be a finitely supported real-valued orthonormal $2 I_{d}$-wavelet filter. Let $P=[1,0, \ldots, 0]$. For some $\mathrm{c} \in \frac{1}{2} \mathbb{Z}^{d}$, if $a(2 \mathrm{c}-k)=a(k)$ for all $k \in \mathbb{Z}^{d}$ and the filter $a$ is projectable with respect to $\left(P, 2 I_{d}, 2\right)$, then $\operatorname{sm}_{p}\left(a, 2 I_{d}\right) \leqslant 1 / p$ for all $1 \leqslant p \leqslant \infty, \operatorname{sr}\left(a, 2 I_{d}\right) \leqslant 1$, and $P a$ must be a shifted version of the Haar filter $a_{H}:=\{1 / 2,1 / 2\}_{[0,1]}$.

By Theorem 2.1, $P a$ is a real-valued orthonormal 2-wavelet filter having symmetry. This forces $P a$ to be a shifted version of the Haar filter ([4]) and now all the claims follow directly from Theorem 3.12. In fact, though many multidimensional filters may be nonseparable (that is, not tensor product filters), they however are often projectable. For example, a filter $a$ is projectable with respect to $\left([1,0, \ldots, 0], 2 I_{d}, 2\right)$ if one of the following conditions holds:

(1) $\widehat{a}$ contains a projectable factor such as $\prod_{j=1}^{d}\left(1+e^{-i 2 \pi \xi_{j}}\right)$, e.g., this is true if $a(E(k-\mathrm{c})+\mathrm{c})=a(k)$ for all $k \in \mathbb{Z}^{d}$ and all $E=\operatorname{diag}( \pm 1, \ldots, \pm 1)$ with $\mathrm{c}=(1 / 2, \ldots, 1 / 2)$ (see [13, Theorem 4.3]);

(2) $a$ is a filter on $\mathbb{Z}^{d}$ such that $\operatorname{sr}\left(a, 2 I_{d}\right) \geqslant m$ and $a$ is supported inside $[n, n+m] \times \mathbb{Z}^{d-1}$ for some $n \in \mathbb{Z}$ (see [17, Theorem 3.2]);

(3) $a$ is an interpolatory $2 I_{d}$-wavelet filter such that $\operatorname{sr}\left(a, 2 I_{d}\right) \geqslant 2 m$ and $a$ is supported inside $[1-2 m, 2 m-$ $1] \times \mathbb{Z}^{d-1}($ see $[17$, Theorem 3.2]);

(4) $a$ is an orthonormal $2 I_{d}$-wavelet filter such that $\operatorname{sr}\left(a, 2 I_{d}\right) \geqslant m$ and $a$ is supported inside $[n, n+2 m-$ $1] \times \mathbb{Z}^{d-1}$ for some $n \in \mathbb{Z}$ (see [17, Theorem 3.2]).

Example 3.14. ([17, Proposition 3.4]) Let $a \in l_{0}\left(\mathbb{Z}^{d}\right)$ be a filter such that $\operatorname{sr}\left(a, 2 I_{d}\right) \geqslant 4$ and $a$ vanishes outside the set $[-2,2] \times \mathbb{Z}^{d-1}$ (one such example is the box spline filter $a$ for the Loop subdivision scheme given by $\left.\widehat{a}\left(\xi_{1}, \xi_{2}\right):=2^{-6}\left(1+e^{-i 2 \pi \xi_{1}}\right)^{2}\left(1+e^{-i 2 \pi \xi_{2}}\right)^{2}\left(1+e^{i 2 \pi\left(\xi_{1}+\xi_{2}\right)}\right)^{2}\right)$. Then there does not exist a dual filter $\tilde{a}$ of $a$ such that the support of $\tilde{a}$ can be supported inside $[-4,4] \times \mathbb{Z}^{d-1}$ and $\operatorname{sm}_{2}\left(\tilde{a}, 2 I_{d}\right)>0$.

Let $P=[1,0, \ldots, 0]$. By item (2) of Example 3.13, the above condition implies that $a$ is projectable with respect to $\left(P, 2 I_{d}, 2\right)$. If there exists such a dual $2 I_{d}$-wavelet filter $\tilde{a}$, then $(P \tilde{a}, P a)$ must be a pair of biorthogonal 2-wavelet filters. However, we must have $\widehat{P a}(\xi)=e^{i 4 \pi \xi}\left(1+e^{-i 2 \pi \xi}\right)^{4} / 16$ and there does not exist such a dual 2-wavelet filter $u$ to $P a$ such that $u$ is supported inside $[-4,4]$ and $\operatorname{sm}_{2}(u, 2)>0$. A dual $2 I_{2}$-wavelet filter $\tilde{a}$ of the box spline $a$ for the Loop subdivision scheme is reported in [17, Example 3.5] such that $\tilde{a}$ is supported inside $[-5,5]^{2}$, is $D_{6}$-symmetric, and $\operatorname{sm}_{2}\left(\tilde{a}, 2 I_{2}\right) \approx 0.10707$.

We complete this section by an example of two-dimensional orthonormal $2 I_{2}$-wavelet filters.

Example 3.15. The following orthonormal $2 I_{2}$-wavelet filter a having support $[0,3]^{2}$ is given in $[20$, Example 4.5]:

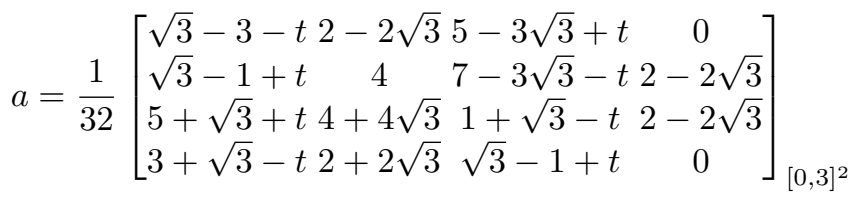

with $t:=\sqrt{6 \sqrt{3}-10}$. Let $\left\{a ; b_{1}, b_{2}, b_{3}\right\}$ be an orthonormal $2 I_{2}$-wavelet filter bank derived from $a$. By item (4) of Example 3.13 and $\operatorname{sr}\left(a, 2 I_{2}\right)=2, a$ is projectable with respect to $\left(P, 2 I_{2}, 2\right)$, where $P:=[1,0]$. Indeed, $P a=\frac{1}{8}\{1+\sqrt{3}, 3+\sqrt{3}, 3-\sqrt{3}, 1-\sqrt{3}\}_{[0,3]}$ is the Daubechies orthonormal 2-wavelet filter $([4])$ and $\left\{P a ; P b_{1}, P b_{2}, P b_{3}\right\}$ is a tight 2-framelet filter bank. By calculation, $\operatorname{sm}_{2}\left(a, 2 I_{2}\right)=\operatorname{sm}_{2}(P a, 2)=1$. Hence, $a$ is an optimal orthonormal $2 I_{2}$-wavelet filter. This example shows that nonseparable orthonormal filters may have smaller support while achieve the same smoothness exponent and sum rules as the corresponding tensor product orthonormal filters. Let $Q:=[1,1]$. Then the condition in (3.2) is also satisfied. Therefore, $\left\{Q a ; Q b_{1}, Q b_{2}, Q b_{3}\right\}$ is a tight 2-framelet filter bank. By calculation, we have $\operatorname{sm}_{2}(Q a, 2) \approx 1.98482$ and $\operatorname{sr}(Q a, 2)=2$. Therefore, $\operatorname{sm}_{2}\left(a, 2 I_{2}\right)<\operatorname{sm}_{2}(Q a, 2)$. 


\section{Frequency-based Affine Systems and Dual Framelets by the Projection Method}

In previous sections we have studied the projection method for constructing and analyzing multidimensional filters and refinable functions. In this section, we shall investigate the application of the projection method for frequency-based affine systems and frequency-based dual framelets.

Following the standard notation, we denote by $\mathscr{D}\left(\mathbb{R}^{d}\right)$ the linear space of all compactly supported $\mathscr{C}^{\infty}$ (test) functions on $\mathbb{R}^{d}$. For $1 \leqslant p<\infty$, by $L_{p}^{\text {loc }}\left(\mathbb{R}^{d}\right)$ we denote the linear space of all Lebesgue measurable functions $f$ such that $\int_{K}|f(x)|^{p} d x<\infty$ for every compact subset $K$ of $\mathbb{R}^{d}$. For $\mathbf{f} \in \mathscr{D}\left(\mathbb{R}^{d}\right)$ and $\boldsymbol{\psi} \in L_{1}^{\text {loc }}\left(\mathbb{R}^{d}\right)$, we shall use the following pairing

$$
\langle\mathbf{f}, \boldsymbol{\psi}\rangle:=\int_{\mathbb{R}^{d}} \mathbf{f}(\xi) \overline{\boldsymbol{\psi}(\xi)} d \xi \quad \text { and } \quad\langle\boldsymbol{\psi}, \mathbf{f}\rangle:=\overline{\langle\mathbf{f}, \boldsymbol{\psi}\rangle}=\int_{\mathbb{R}^{d}} \boldsymbol{\psi}(\xi) \overline{\mathbf{f}(\xi)} d \xi .
$$

Let $J$ be an integer and $\mathrm{M}_{j}, j \geqslant J$ be $d \times d$ invertible real-valued matrices. Let $\boldsymbol{\Phi}$ and $\boldsymbol{\Psi}_{j}, j \geqslant J$ be finite subsets of $L_{2}^{\text {loc }}\left(\mathbb{R}^{d}\right)$. A frequency-based affine system is defined to be

$$
\begin{aligned}
\operatorname{FAS}_{J}\left(\boldsymbol{\Phi} ;\left\{\boldsymbol{\Psi}_{j} \mid \mathrm{M}_{j}\right\}_{j=J}^{\infty}\right)= & \left\{\boldsymbol{\varphi}_{\left(\mathrm{M}_{J}^{\top}\right)^{-1} ; 0, k}: k \in \mathbb{Z}^{d}, \boldsymbol{\varphi} \in \boldsymbol{\Phi}\right\} \\
& \cup \cup_{j=J}^{\infty}\left\{\boldsymbol{\psi}_{\left(\mathrm{M}_{j}^{\top}\right)^{-1} ; 0, k}: k \in \mathbb{Z}^{d}, \boldsymbol{\psi} \in \boldsymbol{\Psi}_{j}\right\} .
\end{aligned}
$$

For the particular case $\mathrm{M}_{j}=\mathrm{M}^{j}$ and $\boldsymbol{\Psi}_{j}=\boldsymbol{\Psi}$ for all $j \geqslant J$, a frequency-based affine system in (4.2) becomes a frequency-based stationary $\mathrm{M}$-affine system:

$$
\operatorname{FAS}_{J}^{\mathrm{M}}(\boldsymbol{\Phi} ; \boldsymbol{\Psi}):=\left\{\boldsymbol{\varphi}_{\left(\mathrm{M}^{\top}\right)^{-J} ; 0, k}: k \in \mathbb{Z}^{d}, \boldsymbol{\varphi} \in \boldsymbol{\Phi}\right\} \cup \bigcup_{j=J}^{\infty}\left\{\boldsymbol{\psi}_{\left(\mathrm{M}^{\top}\right)^{-j} ; 0, k}: k \in \mathbb{Z}^{d}, \boldsymbol{\psi} \in \boldsymbol{\Psi}\right\} .
$$

We now recall the definition of frequency-based dual framelets introduced in $[9,10]$. Let

$$
\boldsymbol{\Phi}=\left\{\boldsymbol{\varphi}^{[1]}, \ldots, \boldsymbol{\varphi}^{[r]}\right\} \quad \text { and } \quad \tilde{\boldsymbol{\Phi}}=\left\{\tilde{\boldsymbol{\varphi}}^{[1]}, \ldots, \tilde{\boldsymbol{\varphi}}^{[r]}\right\}
$$

and

$$
\boldsymbol{\Psi}_{j}=\left\{\boldsymbol{\psi}^{[j, 1]}, \ldots, \boldsymbol{\psi}^{\left[j, s_{j}\right]}\right\} \quad \text { and } \quad \tilde{\boldsymbol{\Psi}}_{j}=\left\{\tilde{\boldsymbol{\psi}}^{[j, 1]}, \ldots, \tilde{\boldsymbol{\psi}}^{\left[j, s_{j}\right]}\right\}
$$

be finite subsets of $L_{2}^{\text {loc }}\left(\mathbb{R}^{d}\right)$ for all integers $j \geqslant J$ with $r, s \in \mathbb{N}_{0}$. Let $\operatorname{FAS}_{J}\left(\boldsymbol{\Phi} ;\left\{\boldsymbol{\Psi}_{j} \mid \mathrm{M}_{j}\right\}_{j=J}^{\infty}\right)$ be defined in (4.2) and $\operatorname{FAS}_{J}\left(\tilde{\boldsymbol{\Phi}} ;\left\{\tilde{\boldsymbol{\Psi}}_{j} \mid \mathrm{M}_{j}\right\}_{j=J}^{\infty}\right)$ be defined similarly. We say $([9,10])$ that the pair

$$
\left(\operatorname{FAS}_{J}\left(\boldsymbol{\Phi} ;\left\{\boldsymbol{\Psi}_{j} \mid \mathrm{M}_{j}\right\}_{j=J}^{\infty}\right), \operatorname{FAS}_{J}\left(\tilde{\boldsymbol{\Phi}} ;\left\{\tilde{\boldsymbol{\Psi}}_{j} \mid \mathrm{M}_{j}\right\}_{j=J}^{\infty}\right)\right)
$$

is a pair of frequency-based nonstationary dual $\left\{\mathrm{M}_{j}\right\}_{j=J}^{\infty}$-framelets if for every $\mathbf{f}, \mathbf{g} \in \mathscr{D}\left(\mathbb{R}^{d}\right)$, the following limit exists:

$$
\begin{aligned}
\lim _{J^{\prime} \rightarrow+\infty}\left(\sum_{\ell=1}^{r} \sum_{\mathrm{k} \in \mathbb{Z}^{d}}\left\langle\mathbf{f}, \boldsymbol{\varphi}_{\left(\mathrm{M}_{J}^{\top}\right)^{-1} ; 0, k}^{[\ell]}\right\rangle\left\langle\tilde{\boldsymbol{\varphi}}_{\left(\mathrm{M}_{J}^{\top}\right)^{-1} ; 0, k}^{[\ell]}, \mathbf{g}\right\rangle\right. \\
\left.\left.\quad+\sum_{j=J}^{J^{\prime}} \sum_{\ell=1}^{s_{j}} \sum_{k \in \mathbb{Z}^{d}}\left\langle\mathbf{f}, \boldsymbol{\psi}_{\left(\mathrm{M}_{j}^{\top}\right)^{-1} ; 0, k}^{[j, \ell]}\right\rangle \tilde{\boldsymbol{\psi}}_{\left(\mathrm{M}_{j}^{\top}\right)^{-1} ; 0, k}^{[j, \ell]}, \mathbf{g}\right\rangle\right)=\langle\mathbf{f}, \mathbf{g}\rangle .
\end{aligned}
$$

Since all $\boldsymbol{\Phi}, \tilde{\boldsymbol{\Phi}}, \boldsymbol{\Psi}_{j}, \tilde{\boldsymbol{\Psi}}_{j}, j \geqslant J$ are finite subsets of $L_{2}^{\text {loc }}\left(\mathbb{R}^{d}\right)$, as shown in [9, 10], the series/summations $\sum_{k \in \mathbb{Z}^{d}}$ on the left-hand side of (4.7) converge absolutely. Consequently, we often write (4.7) as

$$
\sum_{\ell=1}^{r} \sum_{k \in \mathbb{Z}^{d}}\left\langle\mathbf{f}, \varphi_{\left(\mathrm{M}_{J}^{\top}\right)^{-1} ; 0, k}^{[\ell]}\right\rangle\left\langle\tilde{\boldsymbol{\varphi}}_{\left(\mathrm{M}_{J}^{\top}\right)^{-1} ; 0, k}^{[\ell]}, \mathbf{g}\right\rangle+\sum_{j=J}^{\infty} \sum_{\ell=1}^{s_{j}} \sum_{k \in \mathbb{Z}^{d}}\left\langle\mathbf{f}, \boldsymbol{\psi}_{\left(\mathrm{M}_{j}^{\top}\right)^{-1} ; 0, k}^{[j, \ell]}\right\rangle\left\langle\tilde{\boldsymbol{\psi}}_{\left(\mathrm{M}_{j}^{\top}\right)^{-1} ; 0, k}^{[j, \ell]}, \mathbf{g}\right\rangle=\langle\mathbf{f}, \mathbf{g}\rangle
$$


for all $\mathbf{f}, \mathbf{g} \in \mathscr{D}\left(\mathbb{R}^{d}\right)$, where the convergence of the summation $\sum_{j=J}^{\infty}$ is in the sense of (4.7). In particular, we say that $(\{\boldsymbol{\Phi} ; \boldsymbol{\Psi}\},\{\tilde{\boldsymbol{\Phi}} ; \tilde{\boldsymbol{\Psi}}\})$ is a frequency-based dual $\mathrm{M}$-framelet if $\left(\operatorname{FAS}_{0}^{\mathrm{M}}(\boldsymbol{\Phi} ; \boldsymbol{\Psi}), \operatorname{FAS}_{0}^{\mathrm{M}}(\tilde{\boldsymbol{\Phi}} ; \tilde{\boldsymbol{\Psi}})\right)$ is a pair of frequency-based stationary dual framelets.

Let $P$ be a $d \times D$ projection matrix. For a continuous function $\mathbf{f}: \mathbb{R}^{D} \rightarrow \mathbb{C}$, we define the frequencybased projection operator $\widehat{P}$ by

$$
\widehat{P} \mathbf{f}(\xi):=\mathbf{f}\left(P^{\top} \xi\right), \quad \xi \in \mathbb{R}^{d} .
$$

If $f \in L_{1}\left(\mathbb{R}^{D}\right)$, it follows from (1.1) and (4.9) that $\widehat{P f}=\widehat{P} \widehat{f}$. We have the following result on the projection method for frequency-based nonstationary dual framelets.

Theorem 4.1. Let $\mathrm{N}_{j}, j \geqslant J$ be $D \times D$ invertible real-valued matrices such that

$$
\Lambda_{\mathrm{N}}:=\bigcup_{j=J}^{\infty}\left[\mathrm{N}_{j}^{\top} \mathbb{Z}^{D}\right] \text { has no accumulation point }
$$

and

$$
\left\{j \in \mathbb{Z}: j \geqslant J,\left(\mathbf{N}_{j}^{\top}\right)^{-1} k \in \mathbb{Z}^{D}\right\} \quad \text { is a finite set for every } k \in \Lambda_{\mathbf{N}} \backslash\{0\} .
$$

Let $\mathrm{M}_{j}, j \geqslant J$ be $d \times d$ invertible real-valued matrices with $d \leqslant D$. Define $\Lambda_{\mathrm{M}}:=\cup_{j=J}^{\infty}\left[\mathrm{M}_{j}^{\top} \mathbb{Z}^{d}\right]$. Let $P, P_{j}, j \geqslant J$ be $d \times D$ real-valued projection matrices such that

(1) all projection matrices $P_{j}$ are integer matrices and $P_{j} \mathrm{~N}_{j}=\mathrm{M}_{j} P$ for all $j \geqslant J$;

(2) for every $k \in \Lambda_{\mathrm{M}} \backslash\{0\}$ and $j \geqslant J,\left(\mathrm{M}_{j}^{\top}\right)^{-1} k \in \mathbb{Z}^{d}$ if and only if $\left(\mathrm{N}_{j}^{\top}\right)^{-1} P^{\top} k \in \mathbb{Z}^{D}$.

Let $\boldsymbol{\Phi}, \tilde{\boldsymbol{\Phi}}, \boldsymbol{\Psi}_{j}, \tilde{\boldsymbol{\Psi}}_{j}, j \geqslant J$ in (4.4) and (4.5) be finite subsets of continuous functions on $\mathbb{R}^{D}$. Assume that $\left\{H_{\mathrm{N}, j}\right\}_{j=J}^{\infty}$ converges uniformly on every compact subset of $\mathbb{R}^{D}$, where

$$
H_{\mathrm{N}, j}(\zeta):=\sum_{\ell=1}^{r} \overline{\boldsymbol{\varphi}^{[\ell]}\left(\left(\mathrm{N}_{J}^{\top}\right)^{-1} \zeta\right)} \tilde{\boldsymbol{\varphi}}^{[\ell]}\left(\left(\mathrm{N}_{J}^{\top}\right)^{-1} \zeta\right)+\sum_{n=J}^{j} \sum_{\ell=1}^{s_{n}} \overline{\boldsymbol{\psi}^{[n, \ell]}\left(\left(\mathrm{N}_{n}^{\top}\right)^{-1} \zeta\right)} \tilde{\boldsymbol{\psi}}^{[n, \ell]}\left(\left(\mathrm{N}_{n}^{\boldsymbol{\top}}\right)^{-1} \zeta\right), \quad \zeta \in \mathbb{R}^{D}
$$

If

$$
\left(\operatorname{FAS}_{J}\left(\boldsymbol{\Phi} ;\left\{\boldsymbol{\Psi}_{j} \mid \mathbf{N}_{j}\right\}_{j=J}^{\infty}\right), \operatorname{FAS}_{J}\left(\tilde{\boldsymbol{\Phi}} ;\left\{\tilde{\boldsymbol{\Psi}}_{j} \mid \mathbf{N}_{j}\right\}_{j=J}^{\infty}\right)\right)
$$

is a pair of frequency-based nonstationary dual $\left\{\mathrm{N}_{j}\right\}_{j=J}^{\infty}$-framelets on $\mathbb{R}^{D}$, then

$$
\left(\mathrm{FAS}_{J}\left(\widehat{P_{J}} \boldsymbol{\Phi} ;\left\{\widehat{P_{j}} \boldsymbol{\Psi}_{j} \mid \mathrm{M}_{j}\right\}_{j=J}^{\infty}\right), \mathrm{FAS}_{J}\left(\widehat{P_{J}} \tilde{\boldsymbol{\Phi}} ;\left\{\widehat{P}_{j} \tilde{\boldsymbol{\Psi}}_{j} \mid \mathrm{M}_{j}\right\}_{j=J}^{\infty}\right)\right)
$$

is a pair of frequency-based nonstationary dual $\left\{\mathrm{M}_{j}\right\}_{j=J}^{\infty}$-framelets on $\mathbb{R}^{d}$.

Proof. Under the conditions in (4.10) and (4.11), by [9, Theorem 11], (4.12) is a pair of frequency-based nonstationary dual framelets if and only if

$$
\lim _{j \rightarrow+\infty}\left\langle H_{\mathrm{N}, j}, \mathbf{h}\right\rangle=\langle 1, \mathbf{h}\rangle \quad \forall \mathbf{h} \in \mathscr{D}\left(\mathbb{R}^{D}\right)
$$

and

$$
\mathcal{I}_{\boldsymbol{\Phi}}^{\left(\mathrm{N}_{J}^{\top}\right)^{-1} k}\left(\left(\mathrm{~N}_{J}^{\top}\right)^{-1} \zeta\right)+\sum_{j=J}^{\infty} \mathcal{I}_{\boldsymbol{\Psi}_{j}}^{\left(\mathrm{N}_{j}^{\top}\right)^{-1} k}\left(\left(\mathbf{N}_{j}^{\top}\right)^{-1} \zeta\right)=0, \quad \forall k \in \Lambda_{\mathbf{N}} \backslash\{0\}
$$

for almost every $\zeta \in \mathbb{R}^{D}$, where

$$
\begin{aligned}
& \mathcal{I}_{\boldsymbol{\Phi}}^{k}(\zeta):=\sum_{\ell=1}^{r} \overline{\boldsymbol{\varphi}^{[\ell]}(\zeta)} \tilde{\boldsymbol{\varphi}}^{[\ell]}(\zeta+k), \quad k \in \mathbb{Z}^{D} \quad \text { and } \quad \mathcal{I}_{\boldsymbol{\Phi}}^{k}(\zeta):=0, \quad k \in \mathbb{R}^{D} \backslash \mathbb{Z}^{D}, \\
& \mathcal{I}_{\boldsymbol{\Psi}_{j}}^{k}(\zeta):=\sum_{\ell=1}^{s_{j}} \overline{\boldsymbol{\psi}^{[j, \ell]}(\zeta)} \tilde{\boldsymbol{\psi}}^{[j, \ell]}(\zeta+k), \quad k \in \mathbb{Z}^{D} \quad \text { and } \quad \mathcal{I}_{\boldsymbol{\Psi}_{j}}^{k}(\zeta):=0, \quad k \in \mathbb{R}^{D} \backslash \mathbb{Z}^{D} .
\end{aligned}
$$


Due to our assumption in (4.11), the infinite summation in (4.15) is in fact finite. Since all involved functions in (4.15) are continuous, we conclude that (4.15) holds for all $\zeta \in \mathbb{R}^{D}$ (instead of a.e. $\zeta \in \mathbb{R}^{D}$ ).

By item (1), we see that $P_{j}^{\top} \mathbb{Z}^{d} \subseteq \mathbb{Z}^{D}$ and $P^{\top}\left(\mathrm{M}_{j}^{\top} \mathbb{Z}^{d}\right)=\mathrm{N}_{j}^{\top} P_{j}^{\top} \mathbb{Z}^{d} \subseteq \mathrm{N}_{j}^{\top} \mathbb{Z}^{D}$. Therefore, we must have $P^{\top} \Lambda_{\mathrm{M}} \subseteq \Lambda_{\mathrm{N}}$. Since $P$ has full rank, the mapping $P^{\top}: \Lambda_{\mathrm{M}} \rightarrow \Lambda_{\mathrm{N}}$ with $\gamma \mapsto P^{\top} \gamma$ is one-to-one. We now conclude from (4.10) that $\Lambda_{\mathrm{M}}$ has no accumulation points as well. Similarly, for every $k \in \Lambda_{\mathrm{M}} \backslash\{0\}$, noting that $P^{\top} k \in \Lambda_{\mathrm{N}} \backslash\{0\}$ and $P_{j}^{\top}\left(\mathrm{M}_{j}^{\top}\right)^{-1}=\left(\mathrm{N}_{j}^{\top}\right)^{-1} P^{\top}$, we have

$$
\left\{j \in \mathbb{Z}: j \geqslant J,\left(\mathrm{M}_{j}^{\top}\right)^{-1} k \in \mathbb{Z}^{d}\right\} \subseteq\left\{j \geqslant J: P_{j}^{\top}\left(\mathrm{M}_{j}^{\top}\right)^{-1} k \in \mathbb{Z}^{D}\right\}=\left\{j \geqslant J:\left(\mathrm{N}_{j}^{\top}\right)^{-1} P^{\top} k \in \mathbb{Z}^{D}\right\},
$$

from which and (4.11) we see that $\left\{j \in \mathbb{Z}: j \geqslant J,\left(\mathrm{M}_{j}^{\top}\right)^{-1} k \in \mathbb{Z}^{d}\right\}$ must be a finite set for every $k \in \Lambda_{\mathrm{M}} \backslash\{0\}$. Hence, the similar technical conditions as in (4.10) and (4.11) are satisfied for $\mathrm{M}_{j}, j \geqslant J$.

Let $k \in \Lambda_{\mathrm{M}} \backslash\{0\}$. If $\left(\mathrm{M}_{j}^{\top}\right)^{-1} k \in \mathbb{Z}^{d}$, by definition in (4.17) and item (1) we have

$$
\begin{aligned}
& \mathcal{I}_{\widehat{P}_{j} \Psi_{j}}^{\left(\mathrm{M}_{j}^{\top}\right)^{-1} k}\left(\left(\mathrm{M}_{j}^{\boldsymbol{\top}}\right)^{-1} \xi\right)=\sum_{\ell=1}^{s_{j}} \overline{\widehat{P_{j}} \boldsymbol{\psi}^{[j, \ell]}\left(\left(\mathrm{M}_{j}^{\boldsymbol{\top}}\right)^{-1} \xi\right)} \widehat{P_{j}} \tilde{\psi}^{[j, \ell]}\left(\left(\mathrm{M}_{j}^{\boldsymbol{\top}}\right)^{-1} \xi+\left(\mathrm{M}_{j}^{\boldsymbol{\top}}\right)^{-1} k\right) \\
& =\sum_{\ell=1}^{s_{j}} \overline{\boldsymbol{\psi}^{[j, \ell]}\left(P_{j}^{\top}\left(\mathrm{M}_{j}^{\top}\right)^{-1} \xi\right)} \tilde{\boldsymbol{\psi}}^{[j, \ell]}\left(P_{j}^{\top}\left(\mathrm{M}_{j}^{\boldsymbol{\top}}\right)^{-1} \xi+P_{j}^{\top}\left(\mathrm{M}_{j}^{\boldsymbol{\top}}\right)^{-1} k\right) \\
& =\sum_{\ell=1}^{s_{j}} \overline{\boldsymbol{\psi}^{[j, \ell]}\left(\left(\mathrm{N}_{j}^{\top}\right)^{-1} P^{\top} \xi\right)} \tilde{\boldsymbol{\psi}}^{[j, \ell]}\left(\left(\mathrm{N}_{j}^{\top}\right)^{-1} P^{\top} \xi+\left(\mathrm{N}_{j}^{\top}\right)^{-1} P^{\top} k\right) .
\end{aligned}
$$

Since $P^{\top} \Lambda_{\mathrm{M}} \subseteq \Lambda_{\mathrm{N}}$ and $\left(\mathrm{N}_{j}^{\top}\right)^{-1} P^{\top} k=P_{j}^{\top}\left(\mathrm{M}_{j}^{\top}\right)^{-1} k \in \mathbb{Z}^{D}$, we have $P^{\top} k \in \Lambda_{\mathrm{N}}$. Therefore, we conclude that

$$
\mathcal{I}_{\widehat{P}_{j} \Psi_{j}}^{\left(\mathrm{M}_{j}^{\top}\right)^{-1} k}\left(\left(\mathrm{M}_{j}^{\top}\right)^{-1} \xi\right)=\mathcal{I}_{\boldsymbol{\Psi}_{j}}^{\left(\mathrm{N}_{j}^{\top}\right)^{-1} P^{\top} k}\left(\left(\mathrm{~N}_{j}^{\top}\right)^{-1} P^{\top} \xi\right), \quad \forall \xi \in \mathbb{R}^{d} .
$$

If $\left(\mathrm{M}_{j}^{\top}\right)^{-1} k \notin \mathbb{Z}^{d}$ for $k \in \Lambda_{\mathrm{M}} \backslash\{0\}$, by our assumption in item (2), we see that $\left(\mathrm{N}_{j}^{\top}\right)^{-1} P^{\top} k \notin \mathbb{Z}^{D}$. Consequently, it follows directly from the definition in (4.17) that (4.18) still holds since both sides are identically zero. For every $k \in \Lambda_{\mathrm{M}} \backslash\{0\}$, we now deduce from (4.15) (which holds for every $\zeta \in \mathbb{R}^{D}$ ) that for every $\xi \in \mathbb{R}^{d}$,

$$
\begin{aligned}
\mathcal{I}_{\widehat{P_{J}}}^{\left(\mathrm{M}_{J}^{\top}\right)^{-1} k}\left(\left(\mathrm{M}_{J}^{\top}\right)^{-1} \xi\right)+ & \sum_{j=J}^{\infty} \mathcal{I}_{\widehat{P}_{j} \boldsymbol{\Psi}_{j}}^{\left(\mathrm{M}^{\top}\right)^{-1} k}\left(\left(\mathrm{M}_{j}^{\top}\right)^{-1} \xi\right) \\
& =\mathcal{I}_{\boldsymbol{\Phi}}^{\left(\mathrm{N}_{J}^{\top}\right)^{-1} P^{\top} k}\left(\left(\mathrm{~N}_{J}^{\top}\right)^{-1} P^{\top} \xi\right)+\sum_{j=J}^{\infty} \mathcal{I}_{\boldsymbol{\Psi}_{j}}^{\left(\mathrm{N}_{j}^{\top}\right)^{-1} P^{\top} k}\left(\left(\mathrm{~N}_{j}^{\top}\right)^{-1} P^{\top} \xi\right)=0 .
\end{aligned}
$$

On the other hand, since we assumed that $\left\{H_{\mathrm{N}, j}\right\}_{j=J}^{\infty}$ converges uniformly on every compact subset of $\mathbb{R}^{D}$. It is quite trivial to deduce that (4.14) is equivalent to saying that $\lim _{j \rightarrow \infty} H_{\mathrm{N}, j}(\zeta)=1$. By item (1) we have

$$
\begin{aligned}
H_{\mathrm{M}, j}(\xi) & :=\mathcal{I}_{\widehat{P_{J}} \boldsymbol{\Phi}}^{0}\left(\left(\mathrm{M}_{J}^{\boldsymbol{\top}}\right)^{-1} \xi\right)+\sum_{n=J}^{j} \mathcal{I}_{\widehat{P}_{n} \boldsymbol{\Psi}_{n}}^{0}\left(\left(\mathrm{M}_{n}^{\boldsymbol{\top}}\right)^{-1} \xi\right) \\
& =\mathcal{I}_{\boldsymbol{\Phi}}^{0}\left(P_{J}^{\top}\left(\mathrm{M}_{J}^{\boldsymbol{\top}}\right)^{-1} \xi\right)+\sum_{n=J}^{j} \mathcal{I}_{\boldsymbol{\Psi}_{n}}^{0}\left(P_{n}^{\boldsymbol{\top}}\left(\mathrm{M}_{n}^{\boldsymbol{\top}}\right)^{-1} \xi\right)=H_{\mathrm{N}, j}\left(P^{\boldsymbol{\top}} \xi\right) .
\end{aligned}
$$

Since $\left\{H_{\mathrm{N}, j}\right\}_{j=J}^{\infty}$ converges uniformly to 1 on every compact subset of $\mathbb{R}^{D}$, we conclude that $\left\{H_{\mathrm{M}, j}\right\}_{j=J}^{\infty}$ also converges uniformly to 1 on every compact subset of $\mathbb{R}^{d}$. Consequently, $\lim _{j \rightarrow \infty}\left\langle H_{\mathrm{M}, j}, \mathbf{h}\right\rangle=\langle 1, \mathbf{h}\rangle$ for every $\mathbf{h} \in \mathscr{D}\left(\mathbb{R}^{d}\right)$. Now by $[9$, Theorem 11], we conclude that the pair in (4.13) must be a pair of frequency-based nonstationary dual $\left\{\mathrm{M}_{j}\right\}_{j=J}^{\infty}$-framelets on $\mathbb{R}^{d}$. 
We now study the projection method for a sequence of frequency-based dual framelets.

Theorem 4.2. Let $J_{0}$ be an integer. Let $\mathrm{N}_{j}, j \geqslant J_{0}$ be $D \times D$ invertible real-valued matrices such that

$$
\left\{j \in \mathbb{Z}: j \geqslant J_{0},\left[\left(\mathrm{~N}_{j}^{\top}\right)^{-1} B_{c}(0)\right] \cap \mathbb{Z}^{D} \neq\{0\}\right\} \text { is a finite set for every } c \in[1, \infty),
$$

where $B_{c}(0)$ denotes the ball in $\mathbb{R}^{D}$ with center 0 and radius $c$. Let $\mathrm{M}_{j}, j \geqslant J_{0}$ be $d \times d$ invertible real-valued matrices with $d \leqslant D$. Let $P, P_{j}, j \geqslant J_{0}$ be $d \times D$ real-valued projection matrices such that

(1) all projection matrices $P_{j}$ are integer matrices and $P_{j} \mathrm{~N}_{j}=\mathrm{M}_{j} P$ for all $j \geqslant J_{0}$;

(2) for every $j \geqslant J_{0}$,

$$
P^{\top}\left(\left[\mathrm{M}_{j}^{\top} \mathbb{Z}^{d}\right] \backslash\left[\mathrm{M}_{j+1}^{\top} \mathbb{Z}^{d}\right]\right) \subseteq\left[\mathrm{N}_{j}^{\top} \mathbb{Z}^{D}\right] \backslash\left[\mathrm{N}_{j+1}^{\top} \mathbb{Z}^{D}\right], \quad P^{\top}\left(\left[\mathrm{M}_{j+1}^{\top} \mathbb{Z}^{d}\right] \backslash\left[\mathrm{M}_{j}^{\top} \mathbb{Z}^{d}\right]\right) \subseteq\left[\mathrm{N}_{j+1}^{\top} \mathbb{Z}^{D}\right] \backslash\left[\mathrm{N}_{j}^{\top} \mathbb{Z}^{D}\right]
$$

Let $\boldsymbol{\Psi}_{j}, \tilde{\boldsymbol{\Psi}}_{j}$ as in $(4.5)$ and

$$
\boldsymbol{\Phi}_{j}=\left\{\boldsymbol{\varphi}^{[j, 1]}, \ldots, \boldsymbol{\varphi}^{\left[j, r_{j}\right]}\right\}, \quad \tilde{\boldsymbol{\Phi}}_{j}=\left\{\tilde{\boldsymbol{\varphi}}^{[j, 1]}, \ldots, \tilde{\boldsymbol{\varphi}}^{\left[j, r_{j}\right]}\right\}
$$

be finite subsets of continuous functions on $\mathbb{R}^{D}$. Assume that $\left\{H_{j}\right\}_{j=J_{0}}^{\infty}$ converges uniformly on every compact subset of $\mathbb{R}^{D}$, where $H_{j}(\zeta):=\sum_{\ell=1}^{r_{j}} \overline{\varphi^{[j, \ell]}\left(\left(\mathrm{N}_{j}^{\top}\right)^{-1} \zeta\right)} \tilde{\varphi}^{[j, \ell]}\left(\left(\mathrm{N}_{j}^{\top}\right)^{-1} \zeta\right)$. If the pair

$$
\left(\operatorname{FAS}_{J}\left(\boldsymbol{\Phi}_{J} ;\left\{\boldsymbol{\Psi}_{j} \mid \mathbf{N}_{j}\right\}_{j=J}^{\infty}\right), \operatorname{FAS}_{J}\left(\tilde{\boldsymbol{\Phi}}_{J} ;\left\{\tilde{\boldsymbol{\Psi}}_{j} \mid \mathbf{N}_{j}\right\}_{j=J}^{\infty}\right)\right)
$$

is a pair of frequency-based dual $\left\{\mathrm{N}_{j}\right\}_{j=J}^{\infty}$-framelets on $\mathbb{R}^{D}$ for every $J \geqslant J_{0}$, then the pair

$$
\left(\mathrm{FAS}_{J}\left(\widehat{P}_{J} \boldsymbol{\Phi}_{J} ;\left\{\widehat{P}_{j} \boldsymbol{\Psi}_{j} \mid \mathrm{M}_{j}\right\}_{j=J}^{\infty}\right), \mathrm{FAS}_{J}\left(\widehat{P}_{J} \tilde{\boldsymbol{\Phi}}_{J} ;\left\{\widehat{P}_{j} \tilde{\boldsymbol{\Psi}}_{j} \mid \mathrm{M}_{j}\right\}_{j=J}^{\infty}\right)\right)
$$

is a pair of frequency-based nonstationary dual $\left\{\mathrm{M}_{j}\right\}_{j=J}^{\infty}$-framelets on $\mathbb{R}^{d}$ for every integer $J \geqslant J_{0}$.

Proof. Under the condition in (4.19), it has been shown in [9, Theorem 13] that the pair in (4.22) is a pair of frequency-based nonstationary dual $\left\{\mathrm{N}_{j}\right\}_{j=J}^{\infty}$-framelets on $\mathbb{R}^{D}$ for every integer $J \geqslant J_{0}$ if and only if $\lim _{j \rightarrow+\infty}\left\langle H_{j}, \mathbf{h}\right\rangle=\langle 1, \mathbf{h}\rangle$ for all $\mathbf{h} \in \mathscr{D}\left(\mathbb{R}^{D}\right)$ and for all integers $j \geqslant J_{0}$,

$$
\mathcal{I}_{\boldsymbol{\Phi}_{j}}^{\left(\mathbf{N}_{j}^{\top}\right)^{-1} k}\left(\left(\mathbf{N}_{j}^{\top}\right)^{-1} \zeta\right)+\mathcal{I}_{\boldsymbol{\Psi}_{j}}^{\left(\mathbf{N}_{j}^{\top}\right)^{-1} k}\left(\left(\mathbf{N}_{j}^{\top}\right)^{-1} \zeta\right)=\mathcal{I}_{\boldsymbol{\Phi}_{j+1}}^{\left(\mathbf{N}_{j+1}^{\top}\right)^{-1} k}\left(\left(\mathbf{N}_{j+1}^{\top}\right)^{-1} \zeta\right), \quad \forall k \in\left[\mathbf{N}_{j}^{\top} \mathbb{Z}^{D}\right] \cup\left[\mathbf{N}_{j+1}^{\top} \mathbb{Z}^{D}\right]
$$

for almost every $\zeta \in \mathbb{R}^{D}$, where $\mathcal{I}_{\boldsymbol{\Psi}_{j}}^{k}, k \in \mathbb{R}^{D}$ are defined in (4.17) and

$$
\mathcal{I}_{\boldsymbol{\Phi}_{j}}^{k}(\zeta):=\sum_{\ell=1}^{r_{j}} \overline{\boldsymbol{\varphi}^{[j, \ell]}(\zeta)} \tilde{\boldsymbol{\varphi}}^{[j, \ell]}(\zeta+k), \quad k \in \mathbb{Z}^{D} \quad \text { and } \quad \mathcal{I}_{\boldsymbol{\Phi}_{j}}^{k}(\xi):=0, \quad k \in \mathbb{R}^{D} \backslash \mathbb{Z}^{D}
$$

Since all functions in (4.25) are assumed to be continuous, we see that (4.24) holds for all $\zeta \in \mathbb{R}^{D}$.

For every $c \in[1, \infty)$, since $P_{j}$ is an integer matrix, by item (1) and (4.19) we see that

$$
\begin{aligned}
\left\{j \in \mathbb{Z}: j \geqslant J_{0},\left[\left(\mathrm{M}_{j}^{\top}\right)^{-1} B_{c}(0)\right] \cap \mathbb{Z}^{d} \neq 0\right\} & \subseteq\left\{j \in \mathbb{Z}: j \geqslant J_{0},\left[P_{j}^{\top}\left(\mathrm{M}_{j}^{\top}\right)^{-1} B_{c}(0)\right] \cap \mathbb{Z}^{D} \neq 0\right\} \\
& =\left\{j \in \mathbb{Z}: j \geqslant J_{0},\left[\left(\mathrm{~N}_{j}^{\top}\right)^{-1} P^{\top} B_{c}(0)\right] \cap \mathbb{Z}^{D} \neq 0\right\} \\
& \subseteq\left\{j \in \mathbb{Z}: j \geqslant J_{0},\left[\left(\mathrm{~N}_{j}^{\top}\right)^{-1} B_{c^{\prime}}(0)\right] \cap \mathbb{Z}^{D} \neq 0\right\}
\end{aligned}
$$

is a finite set, where $c^{\prime}$ is a positive number such that $P^{\top} B_{c}(0) \subseteq B_{c^{\prime}}(0)$. Hence, the similar technical condition in (4.19) holds for $\mathrm{M}_{j}, j \geqslant J_{0}$. 
For $n \in \mathrm{M}_{j}^{\top} \mathbb{Z}^{d}$, since $P_{j}$ is an integer matrix, it is trivial to see that $P^{\top} n \in P^{\top} \mathrm{M}_{j}^{\top} \mathbb{Z}^{d}=\mathrm{N}_{j}^{\top} P_{j}^{\top} \mathbb{Z}^{d} \subseteq$ $\mathrm{N}_{j}^{\top} \mathbb{Z}^{D}$ and hence $\left(\mathrm{N}_{j}^{\top}\right)^{-1} P^{\top} n \in \mathbb{Z}^{D}$. Let $k \in\left[\mathrm{M}_{j}^{\top} \mathbb{Z}^{d}\right] \backslash\left[\mathrm{M}_{j+1}^{\top} \mathbb{Z}^{d}\right]$. By definition in (4.17) we have

$$
\begin{aligned}
& \mathcal{I}_{\widehat{P}_{j} \Psi_{j}}^{\left(\mathrm{M}^{\top}\right)^{-1} k}\left(\left(\mathrm{M}_{j}^{\top}\right)^{-1} \xi\right)=\sum_{\ell=1}^{s_{j}} \overline{\widehat{P}_{j}} \boldsymbol{\psi}^{[j, \ell]}\left(\left(\mathrm{M}_{j}^{\top}\right)^{-1} \xi\right) \widehat{P_{j}} \tilde{\boldsymbol{\psi}}^{[j, \ell]}\left(\left(\mathrm{M}_{j}^{\top}\right)^{-1} \xi+\left(\mathrm{M}_{j}^{\top}\right)^{-1} k\right) \\
& =\sum_{\ell=1}^{s_{j}} \overline{\boldsymbol{\psi}^{[j, \ell]}\left(P_{j}^{\top}\left(\mathrm{M}_{j}^{\top}\right)^{-1} \xi\right)} \tilde{\boldsymbol{\psi}}^{[j, \ell]}\left(P_{j}^{\top}\left(\mathrm{M}_{j}^{\top}\right)^{-1} \xi+P_{j}^{\top}\left(\mathrm{M}_{j}^{\top}\right)^{-1} k\right) \\
& =\sum_{\ell=1}^{s_{j}} \overline{\psi^{[j, \ell]}\left(\left(\mathrm{N}_{j}^{\top}\right)^{-1} P^{\top} \xi\right)} \tilde{\boldsymbol{\psi}}^{[j, \ell]}\left(\left(\mathrm{N}_{j}^{\top}\right)^{-1} P^{\top} \xi+\left(\mathrm{N}_{j}^{\top}\right)^{-1} P^{\top} k\right) \\
& =\mathcal{I}_{\Psi_{j}}^{\left(\mathrm{N}_{j}^{\top}\right)^{-1} P^{\top} k}\left(\left(\mathrm{~N}_{j}^{\top}\right)^{-1} P^{\top} \xi\right),
\end{aligned}
$$

where we used $\left(\mathrm{N}_{j}^{\top}\right)^{-1} P^{\top} k \in \mathbb{Z}^{D}$. Similarly, we have $\mathcal{I}_{\widehat{P}_{j} \boldsymbol{\Phi}_{j}}^{\left(\mathrm{M}_{j}^{\top}\right)^{-1} k}\left(\left(\mathrm{M}_{j}^{\top}\right)^{-1} \xi\right)=\mathcal{I}_{\boldsymbol{\Phi}_{j}}^{\left(\mathrm{N}_{j}^{\top}\right)^{-1} P^{\top} k}\left(\left(\mathrm{~N}_{j}^{\top}\right)^{-1} P^{\top} \xi\right)$. Consequently, by (4.24) and the first assumption in (4.20), for all $k \in\left[\mathrm{M}_{j}^{\top} \mathbb{Z}^{d}\right] \backslash\left[\mathrm{M}_{j+1}^{\top} \mathbb{Z}^{d}\right]$, we have

$$
\mathcal{I}_{\widehat{P}_{j} \boldsymbol{\Phi}_{j}}^{\left(\mathrm{M}_{j}^{\top}\right)^{-1} k}\left(\left(\mathrm{M}_{j}^{\top}\right)^{-1} \xi\right)+\mathcal{I}_{\widehat{P}_{j} \boldsymbol{\Psi}_{j}}^{\left(\mathrm{M}_{j}^{\top}\right)^{-1} k}\left(\left(\mathrm{M}_{j}^{\top}\right)^{-1} \xi\right)=\mathcal{I}_{\boldsymbol{\Phi}_{j}}^{\left(\mathrm{N}_{j}^{\top}\right)^{-1} P^{\top} k}\left(\left(\mathrm{~N}_{j}^{\top}\right)^{-1} P^{\top} \xi\right)+\mathcal{I}_{\boldsymbol{\Psi}_{j}}^{\left(\mathrm{N}_{j}^{\top}\right)^{-1} P^{\top} k}\left(\left(\mathrm{~N}_{j}^{\top}\right)^{-1} P^{\top} \xi\right)=0 .
$$

Let $k \in\left[\mathrm{M}_{j+1}^{\top} \mathbb{Z}^{d}\right] \backslash\left[\mathrm{M}_{j}^{\top} \mathbb{Z}^{d}\right]$. By definition in (4.17) we have

$$
\begin{aligned}
\mathcal{I}_{\left.\widehat{P}_{j+1} \boldsymbol{M}_{j+1}^{\top}\right)^{-1} k}^{\left(\mathrm{\Phi}_{j+1}\right.}\left(\left(\mathrm{M}_{j+1}^{\top}\right)^{-1} \xi\right) & =\sum_{\ell=1}^{r_{j+1}} \widehat{\widehat{P_{j+1}} \varphi^{[j+1, \ell]}\left(\left(\mathrm{M}_{j+1}^{\top}\right)^{-1} \xi\right)} \widehat{P_{j+1}} \tilde{\boldsymbol{\varphi}}^{[j+1, \ell]}\left(\left(\mathrm{M}_{j+1}^{\top}\right)^{-1} \xi+\left(\mathrm{M}_{j+1}^{\top}\right)^{-1} k\right) \\
& =\sum_{\ell=1}^{r_{j+1}} \overline{\boldsymbol{\varphi}^{[j+1, \ell]}\left(\left(\mathrm{N}_{j+1}^{\top}\right)^{-1} P^{\top} \xi\right)} \tilde{\varphi}^{[j+1, \ell]}\left(\left(\mathrm{N}_{j+1}^{\top}\right)^{-1} P^{\top} \xi+\left(\mathrm{N}_{j+1}^{\top}\right)^{-1} P^{\top} k\right) \\
& =\mathcal{I}_{\boldsymbol{\Phi}_{j+1}}^{\left(\mathrm{N}_{j+1}^{\top}\right)^{-1} P^{\top} k}\left(\left(\mathrm{~N}_{j+1}^{\top}\right)^{-1} P^{\top} \xi\right)=0,
\end{aligned}
$$

where in the last identity we used (4.24) and the second assumption in (4.20).

Let $k \in\left[\mathrm{M}_{j}^{\top} \mathbb{Z}^{d}\right] \cap\left[\mathrm{M}_{j+1}^{\top} \mathbb{Z}^{d}\right]$. By item (1) we have that $P^{\top} k \in\left[\mathrm{N}_{j}^{\top} \mathbb{Z}^{D}\right] \cap\left[\mathrm{N}_{j+1}^{\top} \mathbb{Z}^{D}\right]$. By a similar calculation, we have

$$
\mathcal{I}_{\widehat{P}_{j} \boldsymbol{\Phi}_{j}}^{\left(\mathrm{M}^{\top}\right)^{-1} k}\left(\left(\mathrm{M}_{j}^{\top}\right)^{-1} \xi\right)=\mathcal{I}_{\boldsymbol{\Phi}_{j}}^{\left(\mathrm{N}_{j}^{\top}\right)^{-1} P^{\top} k}\left(\left(\mathrm{~N}_{j}^{\top}\right)^{-1} P^{\top} \xi\right), \quad \mathcal{I}_{\widehat{P}_{j} \boldsymbol{\Psi}_{j}}^{\left(\mathrm{M}^{\top}\right)^{-1} k}\left(\left(\mathrm{M}_{j}^{\top}\right)^{-1} \xi\right)=\mathcal{I}_{\boldsymbol{\Psi}_{j}}^{\left(\mathrm{N}_{j}^{\top}\right)^{-1} P^{\top} k}\left(\left(\mathrm{~N}_{j}^{\top}\right)^{-1} P^{\top} \xi\right),
$$

and

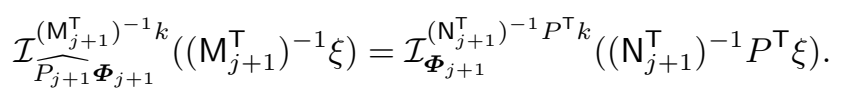

Consequently, it follows from (4.24) that for every $k \in\left[\mathrm{M}_{j}^{\top} \mathbb{Z}^{d}\right] \cap\left[\mathrm{M}_{j+1}^{\top} \mathbb{Z}^{d}\right]$,

$$
\begin{aligned}
\mathcal{I}_{\widehat{P}_{j} \boldsymbol{\Phi}_{j}}^{\left(\mathrm{M}_{j}^{\top}\right)^{-1} k}\left(\left(\mathrm{M}_{j}^{\top}\right)^{-1} \xi\right)+\mathcal{I}_{\widehat{P}_{j} \boldsymbol{\Psi}_{j}}^{\left(\mathrm{M}_{j}^{\top}\right)^{-1} k}\left(\left(\mathrm{M}_{j}^{\top}\right)^{-1} \xi\right) & =\mathcal{I}_{\boldsymbol{\Phi}_{j}}^{\left(\mathrm{N}_{j}^{\top}\right)^{-1} P^{\top} k}\left(\left(\mathrm{~N}_{j}^{\top}\right)^{-1} P^{\top} \xi\right)+\mathcal{I}_{\boldsymbol{\Psi}_{j}}^{\left(\mathrm{N}_{j}^{\top}\right)^{-1} P^{\top} k}\left(\left(\mathrm{~N}_{j}^{\top}\right)^{-1} P^{\top} \xi\right) \\
& =\mathcal{I}_{\boldsymbol{\Phi}_{j+1}}^{\left(\mathrm{N}_{j+1}^{\top}\right)^{-1} P^{\top} k}\left(\left(\mathrm{~N}_{j+1}^{\top}\right)^{-1} P^{\top} \xi\right)=\mathcal{I}_{\widehat{P_{j+1}} \boldsymbol{\Phi}_{j+1}}^{\left(\mathrm{M}_{j+1}^{\top}\right)^{-1} k}\left(\left(\mathrm{M}_{j+1}^{\top}\right)^{-1} \xi\right) .
\end{aligned}
$$

Since $\left\{H_{j}\right\}_{j=J_{0}}^{\infty}$ converges uniformly on every compact subset of $\mathbb{R}^{D}$, we see that $\lim _{j \rightarrow+\infty}\left\langle H_{j}, \mathbf{h}\right\rangle=\langle 1, \mathbf{h}\rangle$ for all $\mathbf{h} \in \mathscr{D}\left(\mathbb{R}^{D}\right)$ if and only if $\left\{H_{j}\right\}_{j=J_{0}}^{\infty}$ converges uniformly to 1 on every compact subset of $\mathbb{R}^{D}$. Therefore, $\left\{H_{j}\left(P^{\top} \cdot\right)\right\}_{j=J_{0}}^{\infty}$ converges uniformly to 1 on every compact subset of $\mathbb{R}^{d}$. As a consequence, we have $\lim _{j \rightarrow+\infty}\left\langle H_{j}\left(P^{\top} \cdot\right), \mathbf{h}\right\rangle=\langle 1, \mathbf{h}\rangle$ for all $\mathbf{h} \in \mathscr{D}\left(\mathbb{R}^{d}\right)$. Now by [9, Theorem 13], we conclude that the pair in (4.23) must be a pair of frequency-based nonstationary dual $\left\{\mathrm{M}_{j}\right\}_{j=J}^{\infty}$-framelets on $\mathbb{R}^{d}$ for every $J \geqslant J_{0}$. 
As a direct consequence of Theorem 4.2, we have

Corollary 4.3. Let $\mathrm{N}$ be a $D \times D$ expansive real-valued matrix and $\mathrm{M}$ be a $d \times d$ invertible real-valued matrix with $d \leqslant D$. Let $P$ be a $d \times D$ integer projection matrix satisfying

$$
P \mathrm{~N}=\mathrm{MP} \quad \text { and } \quad P^{\top}\left(\mathbb{Z}^{d} \backslash\left[\mathrm{M}^{\top} \mathbb{Z}^{d}\right]\right) \subseteq \mathbb{Z}^{D} \backslash\left[\mathrm{N}^{\top} \mathbb{Z}^{D}\right], \quad P^{\top}\left(\left[\mathrm{M}^{\top} \mathbb{Z}^{d}\right] \backslash \mathbb{Z}^{d}\right) \subseteq\left[\mathrm{N}^{\top} \mathbb{Z}^{D}\right] \backslash \mathbb{Z}^{D} .
$$

Let $\boldsymbol{\Phi}, \tilde{\boldsymbol{\Phi}}$ and $\boldsymbol{\Psi}, \tilde{\boldsymbol{\Psi}}$ be finite subsets of continuous functions on $\mathbb{R}^{D}$. If $(\{\boldsymbol{\Phi} ; \boldsymbol{\Psi}\},\{\tilde{\boldsymbol{\Phi}} ; \tilde{\boldsymbol{\Psi}}\})$ is a frequencybased dual $\mathbf{N}$-framelet on $\mathbb{R}^{D}$, then $(\{\widehat{P} \boldsymbol{\Phi} ; \widehat{P} \boldsymbol{\Psi}\},\{\widehat{P} \tilde{\boldsymbol{\Phi}} ; \widehat{P} \tilde{\boldsymbol{\Psi}}\})$ is a frequency-based dual $\mathrm{M}$-framelet on $\mathbb{R}^{d}$.

Proof. As known in $[9$, Corollary 16], since $(\{\boldsymbol{\Phi} ; \boldsymbol{\Psi}\},\{\tilde{\boldsymbol{\Phi}} ; \tilde{\boldsymbol{\Psi}}\})$ is a frequency-based dual N-framelet, $\left(\operatorname{FAS}_{J}^{\mathrm{N}}(\boldsymbol{\Phi} ; \boldsymbol{\Psi}), \operatorname{FAS}_{J}^{\mathrm{N}}(\tilde{\boldsymbol{\Phi}} ; \tilde{\boldsymbol{\Psi}})\right)$ is a pair of frequency-based dual $\mathrm{N}$-framelets for every $J \in \mathbb{Z}$. Set $\mathrm{N}_{j}:=\mathrm{N}^{j}$ and $\mathrm{M}_{j}:=\mathrm{M}^{j}$. Then we can directly check that the condition in (4.26) implies (4.20) in Theorem 4.2. Since $\mathrm{N}$ is expansive, it is easy to directly check that the condition in (4.19) is satisfied. Let $\boldsymbol{\Phi}_{j}:=\boldsymbol{\Phi}, \tilde{\boldsymbol{\Phi}}_{j}:=\tilde{\boldsymbol{\Phi}}$ and $\boldsymbol{\Psi}_{j}:=\boldsymbol{\Psi}, \tilde{\boldsymbol{\Psi}}_{j}:=\tilde{\boldsymbol{\Psi}}$ in Theorem 4.2. Note that $H_{j}(\zeta)=\sum_{\ell=1}^{r} \overline{\varphi^{[\ell]}\left(\left(\mathrm{N}^{\top}\right)^{-j} \zeta\right)} \tilde{\boldsymbol{\varphi}}^{[\ell]}\left(\left(\mathrm{N}^{\top}\right)^{-j} \zeta\right)$. Since all functions in the finite sets $\boldsymbol{\Phi}=\left\{\boldsymbol{\varphi}^{[1]}, \ldots, \boldsymbol{\varphi}^{[r]}\right\}$ and $\tilde{\boldsymbol{\Phi}}=\left\{\tilde{\boldsymbol{\varphi}}^{[1]}, \ldots, \tilde{\boldsymbol{\varphi}}^{[r]}\right\}$ are assumed to be continuous and since $\mathbf{N}$ is expansive, it is quite easy to see that $\left\{H_{j}\right\}_{j=0}^{\infty}$ converges uniformly to $\sum_{\ell=1}^{r} \overline{\varphi^{[\ell]}(0)} \tilde{\varphi}^{[\ell]}(0)$ on every compact subset of $\mathbb{R}^{D}$. Now the claim follows directly from Theorem 4.2. This completes the proof.

As discussed in $[9,10]$, dual framelet filter banks have a close relation to frequency-based dual framelets. This connection naturally links the projection method in Theorem 2.3 for projected dual framelet filter banks and the projection method in Corollary 4.3 for frequency-based dual framelets. For the convenience of the reader, we present such a connection here for a particular simple case.

Theorem 4.4. ([10, Theorem 2] and [9, Theorem 17]) Let $\mathrm{M}$ be a $d \times d$ expansive integer matrix. Let $\theta, a, b_{1}, \ldots, b_{s}, \tilde{\theta}, \tilde{a}, \tilde{b}_{1}, \ldots, \tilde{b}_{s} \in l_{0}\left(\mathbb{Z}^{d}\right)$ such that $\widehat{a}(0)=\widehat{\tilde{a}}(0)=\widehat{\widehat{\theta}}(0) \widehat{\tilde{\theta}}(0)=1$. Define

$$
\varphi(\xi):=\prod_{j=1}^{\infty} \widehat{a}\left(\left(\mathrm{M}^{\top}\right)^{-j} \xi\right) \quad \text { and } \quad \tilde{\varphi}(\xi):=\prod_{j=1}^{\infty} \widehat{\tilde{a}}\left(\left(\mathrm{M}^{\top}\right)^{-j} \xi\right), \quad \xi \in \mathbb{R}^{d}
$$

and $\boldsymbol{\eta}(\xi):=\widehat{\theta}(\xi) \varphi(\xi), \tilde{\eta}(\xi):=\widehat{\tilde{\theta}}(\xi) \tilde{\varphi}(\xi)$. Define

$$
\psi^{[\ell]}\left(\mathrm{M}^{\top} \xi\right):=\widehat{b_{\ell}}(\xi) \varphi(\xi), \quad \tilde{\boldsymbol{\psi}}^{[\ell]}\left(\mathrm{M}^{\top} \xi\right):=\widehat{\tilde{b}}_{\ell}(\xi) \tilde{\varphi}(\xi), \quad \xi \in \mathbb{R}^{d}, \ell=1, \ldots, s .
$$

Then $(\{\boldsymbol{\Phi} ; \boldsymbol{\Psi}\},\{\tilde{\boldsymbol{\Phi}} ; \tilde{\boldsymbol{\Psi}}\})$, with $\boldsymbol{\Phi}:=\{\boldsymbol{\eta}\}, \boldsymbol{\Psi}:=\left\{\boldsymbol{\psi}^{[1]}, \ldots, \boldsymbol{\psi}^{[s]}\right\}, \tilde{\boldsymbol{\Phi}}:=\{\tilde{\boldsymbol{\eta}}\}$ and $\tilde{\boldsymbol{\Psi}}:=\left\{\tilde{\boldsymbol{\psi}}^{[1]}, \ldots, \tilde{\boldsymbol{\psi}}^{[s]}\right\}$, is a frequency-based dual $\mathrm{M}$-framelet if and only if $\left(\left\{a ; b_{1}, \ldots, b_{s}\right\},\left\{\tilde{a} ; \tilde{b}_{1}, \ldots, \tilde{b}_{s}\right\}\right)_{\Theta}$ is a dual $\mathrm{M}$-framelet filter bank, where $\Theta$ is defined to be $\widehat{\Theta}(\xi):=\overline{\widehat{\theta}(\xi)} \widehat{\tilde{\theta}}(\xi)$.

If $\mathrm{N}$ and $\mathrm{M}$ are integer matrices, then it is trivial to see that the condition in (4.26) becomes exactly (2.9). Consequently, the connection in Theorem 4.4 explains that the conditions on the projection matrix in Theorem 2.3 and Corollary 4.3 with $\mathrm{N}$ and $\mathrm{M}$ being integer matrices are the same.

\section{Multidimensional Wavelets and Framelets in $L_{2}\left(\mathbb{R}^{d}\right)$ by the Projection Method}

Since most results on wavelet analysis are often stated in the space domain for the square integrable function space $L_{2}\left(\mathbb{R}^{d}\right)$, built on results in Section 4 , in this section we shall investigate multidimensional wavelets and framelets in $L_{2}\left(\mathbb{R}^{d}\right)$ by the projection method. 
Let $J$ be an integer and $\mathrm{M}_{j}, j \geqslant J$ be $d \times d$ invertible real-valued matrices. Let $\Phi$ and $\Psi_{j}, j \geqslant J$ be finite subsets of $L_{2}\left(\mathbb{R}^{d}\right)$. A nonstationary affine system is defined to be

$$
\operatorname{AS}_{J}\left(\Phi ;\left\{\Psi_{j} \mid \mathrm{M}_{j}\right\}_{j=J}^{\infty}\right)=\left\{\phi_{\mathrm{M}_{J} ; k}: k \in \mathbb{Z}^{d}, \phi \in \Phi\right\} \cup \bigcup_{j=J}^{\infty}\left\{\psi_{\mathrm{M}_{j} ; k}: k \in \mathbb{Z}^{d}, \psi \in \Psi_{j}\right\} .
$$

For the particular case $\mathrm{M}_{j}=\mathrm{M}^{j}$ and $\Psi_{j}=\Psi$ for all $j \geqslant J$, the affine system in (5.1) becomes a stationary M-affine system:

$$
\operatorname{AS}_{J}^{\mathrm{M}}(\Phi ; \Psi):=\left\{\phi_{\mathrm{M}^{J} ; k}: k \in \mathbb{Z}^{d}, \phi \in \Phi\right\} \cup \bigcup_{j=J}^{\infty}\left\{\psi_{\mathrm{M}^{j} ; k}: k \in \mathbb{Z}^{d}, \psi \in \Psi\right\} .
$$

For $f \in L_{1}\left(\mathbb{R}^{d}\right)$, by the notation in (1.6), it is straightforward to check that

$$
\widehat{f_{U ; k, n}}=e^{-i 2 \pi k \cdot n} \widehat{f}_{\left(U^{\top}\right)^{-1} ;-n, k} .
$$

In particular, we have $\widehat{f_{U ; k}}=\widehat{f}_{\left(U^{\top}\right)^{-1} ; 0, k}$. Now it is trivial to observe that

$$
\operatorname{FAS}_{J}\left(\widehat{\Phi} ;\left\{\widehat{\Psi_{j}} \mid \mathrm{M}_{j}\right\}_{j=J}^{\infty}\right)=\left\{\widehat{f}: f \in \mathrm{AS}_{J}\left(\Phi ;\left\{\Psi_{j} \mid \mathrm{M}_{j}\right\}_{j=J}^{\infty}\right)\right\}
$$

and

$$
\operatorname{FAS}_{J}^{\mathrm{M}}(\widehat{\Phi} ; \widehat{\Psi})=\left\{\widehat{f}: f \in \mathrm{AS}_{J}^{\mathrm{M}}(\Phi ; \Psi)\right\} .
$$

That is, the frequency-based affine system $\operatorname{FAS}_{J}\left(\widehat{\Phi} ;\left\{\widehat{\Psi_{j}} \mid \mathrm{M}_{j}\right\}_{j=J}^{\infty}\right)$, which is defined in (4.2), is simply the image of $\mathrm{AS}_{J}\left(\Phi ;\left\{\Psi_{j} \mid \mathrm{M}_{j}\right\}_{j=J}^{\infty}\right)$ under the Fourier transform.

We say that $\operatorname{AS}_{J}\left(\Phi ;\left\{\Psi_{j} \mid \mathrm{M}_{j}\right\}_{j=J}^{\infty}\right)$ is a frame for $L_{2}\left(\mathbb{R}^{d}\right)$ if there exist positive constants $C_{1}$ and $C_{2}$ such that

$$
\begin{array}{r}
C_{1}\|f\|_{L_{2}\left(\mathbb{R}^{d}\right)}^{2} \leqslant \sum_{\phi \in \Phi} \sum_{k \in \mathbb{Z}^{d}}\left|\left\langle f, \phi_{\mathrm{M}_{J} ; k}\right\rangle\right|^{2}+\sum_{j=J}^{\infty} \sum_{\psi \in \Psi_{j}} \sum_{k \in \mathbb{Z}^{d}}\left|\left\langle f, \psi_{\mathrm{M}_{j} ; k}\right\rangle\right|^{2} \leqslant C_{2}\|f\|_{L_{2}\left(\mathbb{R}^{d}\right)}^{2}, \\
\forall f \in L_{2}\left(\mathbb{R}^{d}\right) .
\end{array}
$$

If only the right-hand side inequality in (5.3) holds, then we say that $\mathrm{AS}_{J}\left(\Phi ;\left\{\Psi_{j} \mid \mathrm{M}_{j}\right\}_{j=J}^{\infty}\right)$ is a Bessel sequence in $L_{2}\left(\mathbb{R}^{d}\right)$. If $C_{1}=C_{2}=1$ in (5.3), then we say that $\operatorname{AS}_{J}\left(\Phi ;\left\{\Psi_{j} \mid \mathrm{M}_{j}\right\}_{j=J}^{\infty}\right)$ is a normalized tight frame for $L_{2}\left(\mathbb{R}^{d}\right)$. We say that $\{\Phi ; \Psi\}$ is a tight $\mathrm{M}$-framelet in $L_{2}\left(\mathbb{R}^{d}\right)$ if $\mathrm{AS}_{0}^{\mathrm{M}}(\Phi ; \Psi)$ is a normalized tight frame for $L_{2}\left(\mathbb{R}^{d}\right)$.

We now generalize the projection operator in (1.2) from integrable functions to tempered distributions. Let $f$ be a tempered distribution on $\mathbb{R}^{D}$ such that $\widehat{f}$ is a continuous function on $\mathbb{R}^{D}$ (this requires that $\widehat{f}$ should have no more than polynomial growth). Define $\widehat{P f}$ as in (1.1). Then $\widehat{P f}$ is a continuous function having no more than polynomial growth. Thus, $\widehat{P f}$ can be regarded as a tempered distribution and consequently, $P f$ is a well-defined tempered distribution. In particular, if $\sum_{k \in \mathbb{Z}^{D}}|f(\cdot+k)| \in L_{p}\left([0,1]^{D}\right)$ for some $1 \leqslant p \leqslant \infty$ and if $P$ is a $d \times D$ rational projection matrix (that is, all entries of $P$ are rational numbers), then it has been proved in [11, Proposition 2.1] that $\sum_{n \in \mathbb{Z}^{d}}|P f(\cdot+n)| \in L_{p}\left([0,1]^{d}\right)$.

For nonstationary tight framelets in the space of square integrable functions, we have

Theorem 5.1. Assume the same notation and conditions as in Theorem 4.1 for $\mathrm{N}_{j}, \mathrm{M}_{j}, P, P_{j}, j \geqslant J$. Suppose that $\mathrm{AS}_{J}\left(\Phi ;\left\{\Psi_{j} \mid \mathrm{N}_{j}\right\}_{j=J}^{\infty}\right)$ is a normalized tight frame for $L_{2}\left(\mathbb{R}^{D}\right)$. If all elements in $\widehat{\Phi}, \widehat{\Psi_{j}}, j \geqslant J$ are continuous and $\left\{H_{\mathrm{N}, j}\right\}_{j=J}^{\infty}$ converges uniformly on every compact subset of $\mathbb{R}^{D}$, where

$$
H_{\mathrm{N}, j}(\zeta):=\sum_{\phi \in \Phi}\left|\widehat{\phi}\left(\left(\mathrm{N}_{J}^{\top}\right)^{-1} \zeta\right)\right|^{2}+\sum_{n=J}^{j} \sum_{\psi \in \Psi_{n}}\left|\widehat{\psi}\left(\left(\mathrm{N}_{n}^{\top}\right)^{-1} \zeta\right)\right|^{2},
$$

then $\mathrm{AS}_{J}\left(P_{J} \Phi ;\left\{P_{j} \Psi_{j} \mid \mathrm{M}_{j}\right\}_{j=J}^{\infty}\right)$ is a normalized tight frame for $L_{2}\left(\mathbb{R}^{d}\right)$. 
Proof. It has been shown in [10, Corollary 14] and [9, Corollary 16] that $\mathrm{AS}_{J}\left(\Phi ;\left\{\Psi_{j} \mid \mathrm{N}_{j}\right\}_{j=J}^{\infty}\right)$ is a normalized tight frame for $L_{2}\left(\mathbb{R}^{D}\right)$ if and only if $\left(\operatorname{FAS}_{J}\left(\widehat{\Phi} ;\left\{\widehat{\Psi_{j}} \mid \mathrm{N}_{j}\right\}_{j=J}^{\infty}\right), \operatorname{FAS}_{J}\left(\widehat{\Phi} ;\left\{\widehat{\Psi_{j}} \mid \mathrm{N}_{j}\right\}_{j=J}^{\infty}\right)\right)$ is a pair of frequency-based dual framelets on $\mathbb{R}^{D}$. Now it follows directly from Theorem 4.1 that $\left(\mathrm{FAS}_{J}\left(\widehat{P_{J}} \widehat{\Phi} ;\left\{\widehat{P_{j}} \Psi_{j} \mid \mathrm{M}_{j}\right\}_{j=J}^{\infty}\right), \mathrm{FAS}_{J}\left(\widehat{P_{J}} \widehat{\Phi} ;\left\{\widehat{P_{j}} \widehat{\Psi_{j}} \mid \mathrm{M}_{j}\right\}_{j=J}^{\infty}\right)\right)$ is a pair of frequency-based dual framelets on $\mathbb{R}^{d}$. By $\left[9\right.$, Corollary 16], $\mathrm{AS}_{J}\left(P_{J} \Phi ;\left\{P_{j} \Psi_{j} \mid \mathrm{M}_{j}\right\}_{j=J}^{\infty}\right)$ is a normalized tight frame for $L_{2}\left(\mathbb{R}^{d}\right)$.

Similarly, we have the following result.

Theorem 5.2. Assume the same notation and conditions as in Theorem 4.2 for $\mathrm{N}_{j}, \mathrm{M}_{j}, P, P_{j}, j \geqslant J_{0}$. Suppose that $\mathrm{AS}_{J}\left(\Phi_{J} ;\left\{\Psi_{j} \mid \mathrm{N}_{j}\right\}_{j=J}^{\infty}\right)$ is a normalized tight frame for $L_{2}\left(\mathbb{R}^{D}\right)$ for every $J \geqslant J_{0}$. If all elements in $\widehat{\Phi_{j}}, \widehat{\Psi_{j}}, j \geqslant J_{0}$ are continuous and $\left\{H_{j}\right\}_{j=J_{0}}^{\infty}$ converges uniformly on every compact subset of $\mathbb{R}^{D}$, where $H_{j}(\zeta):=\sum_{\phi \in \Phi_{j}}\left|\widehat{\phi}\left(\left(\mathrm{N}_{j}^{\top}\right)^{-1} \zeta\right)\right|^{2}$, then $\mathrm{AS}_{J}\left(P_{J} \Phi_{J} ;\left\{P_{j} \Psi_{j} \mid \mathrm{M}_{j}\right\}_{j=J}^{\infty}\right)$ is a normalized tight frame for $L_{2}\left(\mathbb{R}^{d}\right)$ for every $J \geqslant J_{0}$.

Corollary 5.3. Let $\mathrm{N}$ be a $D \times D$ expansive real-valued matrix and $\mathrm{M}$ be a $d \times d$ invertible real-valued matrix with $d \leqslant D$. Let $P$ be a $d \times D$ integer projection matrix satisfying (4.26). Let $\Phi$ and $\Psi$ be finite subsets of $L_{2}\left(\mathbb{R}^{D}\right)$ such that all the elements in $\widehat{\Phi}$ and $\widehat{\Psi}$ are continuous. If $\{\Phi ; \Psi\}$ is a tight $\mathrm{N}$-framelet in $L_{2}\left(\mathbb{R}^{D}\right)$, then $\{P \Phi ; P \Psi\}$ is a tight $\mathrm{M}$-framelet in $L_{2}\left(\mathbb{R}^{d}\right)$.

We now discuss dual framelets in $L_{2}\left(\mathbb{R}^{d}\right)$. Let $\mathrm{M}_{j}, j \geqslant J$ be $d \times d$ invertible real-valued matrices. Let

$$
\Phi=\left\{\phi^{[1]}, \ldots, \phi^{[r]}\right\} \quad \text { and } \quad \tilde{\Phi}=\left\{\tilde{\phi}^{[1]}, \ldots, \tilde{\phi}^{[r]}\right\}
$$

and

$$
\Psi_{j}=\left\{\psi^{[j, 1]}, \ldots, \psi^{\left[j, s_{j}\right]}\right\} \quad \text { and } \quad \tilde{\Psi}_{j}=\left\{\tilde{\psi}^{[j, 1]}, \ldots, \tilde{\psi}^{\left[j, s_{j}\right]}\right\}
$$

be finite subsets of $L_{2}\left(\mathbb{R}^{d}\right)$ for all integers $j \geqslant J$ with $r, s \in \mathbb{N}_{0}$. Let $\mathrm{AS}_{J}\left(\Phi ;\left\{\Psi_{j} \mid \mathrm{M}_{j}\right\}_{j=J}^{\infty}\right)$ be defined in (5.1) and $\operatorname{AS}_{J}\left(\tilde{\Phi} ;\left\{\tilde{\Psi}_{j} \mid \mathrm{M}_{j}\right\}_{j=J}^{\infty}\right)$ be defined similarly. We say that the pair

$$
\left(\operatorname{AS}_{J}\left(\Phi ;\left\{\Psi_{j} \mid \mathrm{M}_{j}\right\}_{j=J}^{\infty}\right), \operatorname{AS}_{J}\left(\tilde{\Phi} ;\left\{\tilde{\Psi}_{j} \mid \mathrm{M}_{j}\right\}_{j=J}^{\infty}\right)\right)
$$

is a pair of nonstationary dual $\left\{\mathrm{M}_{j}\right\}_{j=J}^{\infty}$-framelets for $L_{2}\left(\mathbb{R}^{d}\right)$ if

(1) $\mathrm{AS}_{J}\left(\Phi ;\left\{\Psi_{j} \mid \mathrm{M}_{j}\right\}_{j=J}^{\infty}\right)$ is a frame for $L_{2}\left(\mathbb{R}^{d}\right)$ and $\mathrm{AS}_{J}\left(\tilde{\Phi} ;\left\{\tilde{\Psi}_{j} \mid \mathrm{M}_{j}\right\}_{j=J}^{\infty}\right)$ is a frame for $L_{2}\left(\mathbb{R}^{d}\right)$;

(2) for every $f, g \in L_{2}\left(\mathbb{R}^{d}\right)$, the following identity holds:

$$
\sum_{\ell=1}^{r} \sum_{k \in \mathbb{Z}^{d}}\left\langle f, \phi_{\mathrm{M}_{J} ; k}^{[\ell]}\right\rangle\left\langle\tilde{\phi}_{\mathrm{M}_{J} ; k}^{[\ell]}, g\right\rangle+\sum_{j=J}^{\infty} \sum_{\ell=1}^{s_{j}} \sum_{k \in \mathbb{Z}^{d}}\left\langle f, \psi_{\mathrm{M}_{j} ; k}^{[j, \ell]}\right\rangle\left\langle\tilde{\psi}_{\mathrm{M}_{j} ; k}^{[j, \ell]}, g\right\rangle=\langle f, g\rangle
$$

with the series converging absolutely.

In particular, we say that $(\{\Phi ; \Psi\},\{\tilde{\Phi} ; \tilde{\Psi}\})$ is a dual $\mathrm{M}$-framelet in $L_{2}\left(\mathbb{R}^{d}\right)$ if

$$
\left(\operatorname{AS}_{J}\left(\Phi ;\left\{\Psi \mid \mathrm{M}^{j}\right\}_{j=J}^{\infty}\right), \operatorname{AS}_{J}\left(\tilde{\Phi} ;\left\{\tilde{\Psi} \mid \mathrm{M}^{j}\right\}_{j=J}^{\infty}\right)\right)
$$

is a pair of nonstationary dual $\left\{\mathrm{M}^{j}\right\}_{j=J^{-}}^{\infty}$ framelets for $L_{2}\left(\mathbb{R}^{d}\right)$.

Under the additional condition on Bessel sequences in $L_{2}\left(\mathbb{R}^{d}\right)$, similar results hold for dual framelets in $L_{2}\left(\mathbb{R}^{d}\right)$. For simplicity, we only present the simplest case here.

Theorem 5.4. Let $\mathrm{N}$ be a $D \times D$ expansive real-valued matrix and $\mathrm{M}$ be a $d \times d$ invertible real-valued matrix with $d \leqslant D$. Let $P$ be a $d \times D$ integer projection matrix satisfying (4.26). Let $\Phi, \tilde{\Phi}$ and $\Psi, \tilde{\Psi}$ be finite subsets of $L_{2}\left(\mathbb{R}^{D}\right)$ such that all elements in $\widehat{\Phi}, \widehat{\tilde{\Phi}}, \widehat{\Psi}, \widehat{\tilde{\Psi}}$ are continuous. If $(\{\Phi ; \Psi\},\{\tilde{\Phi} ; \tilde{\Psi}\})$ is a dual $\mathrm{N}$-framelet in $L_{2}\left(\mathbb{R}^{D}\right)$ and if both $\mathrm{AS}_{0}^{\mathrm{M}}(P \Phi ; P \Psi)$ and $\mathrm{AS}_{0}^{\mathrm{M}}(P \tilde{\Phi}, P \tilde{\Psi})$ are Bessel sequences in $L_{2}\left(\mathbb{R}^{d}\right)$, then $(\{P \Phi ; P \Psi\},\{P \tilde{\Phi} ; P \tilde{\Psi}\})$ is a dual $\mathrm{M}$-framelet in $L_{2}\left(\mathbb{R}^{d}\right)$. 
Proof. By $\left[9\right.$, Theorem 9], that $(\{\Phi ; \Psi\},\{\tilde{\Phi} ; \tilde{\Psi}\})$ is a dual $\mathrm{N}$-framelet in $L_{2}\left(\mathbb{R}^{D}\right)$ if and only if

(1) both $\operatorname{AS}_{0}^{\mathrm{N}}(\Phi ; \Psi)$ and $\operatorname{AS}_{0}^{\mathrm{N}}(\tilde{\Phi} ; \tilde{\Psi})$ are Bessel sequences in $L_{2}\left(\mathbb{R}^{D}\right)$;

(2) $\left.\operatorname{FAS}_{0}^{\mathrm{N}}(\widehat{\Phi} ; \widehat{\Psi}), \operatorname{FAS}_{0}^{\mathrm{N}}(\widehat{\tilde{\Phi}} ; \widehat{\tilde{\Psi}})\right)$ is a pair of frequency-based dual framelets.

By Corollary 4.3 , we see that $\left(\operatorname{FAS}_{0}^{\mathrm{M}}(\widehat{P} \widehat{\Phi} ; \widehat{P} \widehat{\Psi}), \operatorname{FAS}_{0}^{\mathrm{M}}(\widehat{P} \widehat{\tilde{\Phi}} ; \widehat{P} \widehat{\tilde{\Psi}})\right)$ is a pair of frequency-based dual framelets. Note that $\widehat{P} \widehat{\Psi}=\widehat{P \Psi}$. Since both $\mathrm{AS}_{0}^{\mathrm{M}}(P \Phi ; P \Psi)$ and $\mathrm{AS}_{0}^{\mathrm{M}}(P \tilde{\Phi} ; P \tilde{\Psi})$ are Bessel sequences in $L_{2}\left(\mathbb{R}^{d}\right)$, by $\left[9\right.$, Theorem 9], we see that $(\{P \Phi ; P \Psi\},\{P \tilde{\Phi} ; P \tilde{\Psi}\})$ is a dual M-framelet in $L_{2}\left(\mathbb{R}^{d}\right)$.

So far, we are not clear about whether the extra assumption on Bessel property in Theorem 5.4$\operatorname{AS}_{0}^{\mathrm{M}}(P \Phi ; P \Psi)$ and $\mathrm{AS}_{0}^{\mathrm{M}}(P \tilde{\Phi} ; P \tilde{\Psi})$ are Bessel sequences in $L_{2}\left(\mathbb{R}^{d}\right)$-can be removed or not. But as shown by the following result, the Bessel property often holds.

Theorem 5.5. Let $\mathrm{N}$ be a $D \times D$ expansive integer matrix. Let $\phi$ and $\tilde{\phi}$ be two $r \times 1$ vectors of compactly supported functions in $L_{2}\left(\mathbb{R}^{D}\right)$ such that $\widehat{\phi}\left(\mathrm{N}^{\top} \zeta\right)=\widehat{a}(\zeta) \widehat{\phi}(\zeta)$ and $\widehat{\tilde{\phi}}\left(\mathrm{N}^{\top} \zeta\right)=\widehat{\tilde{a}}(\zeta) \widehat{\tilde{\phi}}(\zeta)$ for all $\zeta \in \mathbb{R}^{D}$ for some $r \times r$ matrices $\widehat{a}$ and $\widehat{\tilde{a}}$ of $\mathbb{Z}^{D}$-periodic trigonometric polynomials in D-variables. Let $\theta, \tilde{\theta}, b_{1}, \ldots, b_{s}, \tilde{b}_{1}, \ldots, \tilde{b}_{s} \in\left(l_{0}\left(\mathbb{Z}^{D}\right)\right)^{r \times r}$ such that $\left(\left\{a ; b_{1}, \ldots, b_{s}\right\},\left\{\tilde{a} ; \tilde{b}_{1}, \ldots, \tilde{b}_{s}\right\}\right)_{\Theta}$ with $\widehat{\Theta}(\zeta):=\overline{\widehat{\theta}}(\zeta) \mathrm{T} \widehat{\tilde{\theta}}(\zeta)$ is a dual $\mathrm{N}$-framelet filter bank. Suppose that

$$
\overline{\widehat{\phi}}(0)^{\top} \widehat{\Theta}(0) \widehat{\tilde{\phi}}(0)=1 \quad \text { and } \quad \widehat{b}_{\ell}(0) \widehat{\phi}(0)=\widetilde{\tilde{b}}_{\ell}(0) \widehat{\tilde{\phi}}(0)=0, \quad \forall \ell=1, \ldots, s .
$$

Define $\psi^{[1]}, \ldots, \psi^{[s]}, \tilde{\psi}^{[1]}, \ldots, \tilde{\psi}^{[s]}$ by

$$
\widehat{\psi^{[\ell]}}\left(\mathrm{N}^{\top} \zeta\right):=\widehat{b_{\ell}}(\zeta) \widehat{\phi}(\zeta) \quad \text { and } \quad \widehat{\tilde{\psi}^{[\ell]}}\left(\mathrm{N}^{\top} \zeta\right):=\widehat{\tilde{b}_{\ell}}(\zeta) \widehat{\tilde{\phi}}(\zeta), \quad \ell=1, \ldots, s
$$

Define $\widehat{\eta}(\zeta):=\widehat{\theta}(\zeta) \widehat{\phi}(\zeta)$ and $\widehat{\tilde{\eta}}(\zeta):=\widehat{\tilde{\theta}}(\zeta) \widehat{\tilde{\phi}}(\zeta)$. Then $(\{\Phi ; \Psi\},\{\tilde{\Phi} ; \tilde{\Psi}\})$, with $\Phi:=\{\eta\}, \tilde{\Phi}:=\{\tilde{\eta}\}$ and $\Psi:=\left\{\psi^{[1]}, \ldots, \psi^{[s]}\right\}, \tilde{\Psi}:=\left\{\tilde{\psi}^{[1]}, \ldots, \tilde{\psi}^{[s]}\right\}$, is a dual $\mathrm{N}$-framelet in $L_{2}\left(\mathbb{R}^{D}\right)$. Let $\mathrm{M}$ be a $d \times d$ invertible integer matrix and let $P$ be a $d \times D$ integer projection matrix satisfying (2.9). Then $\left(\left\{P a ; P b_{1}, \ldots, P b_{s}\right\},\left\{P \tilde{a} ; P \tilde{b}_{1}, \ldots, P \tilde{b}_{s}\right\}\right)_{P \Theta}$ is a dual $\mathrm{M}$-framelet filter bank and $(\{P \Phi ; P \Psi\},\{P \tilde{\Phi} ; P \tilde{\Psi}\})$ is a dual $\mathrm{M}$-framelet in $L_{2}\left(\mathbb{R}^{d}\right)$.

Proof. Since $\phi$ and $\tilde{\phi}$ are vector functions in $L_{2}\left(\mathbb{R}^{D}\right)$, by[16, Theorems 2.2 and 2.3] or [11, Theorems 3.2 and 3.4] and (5.7), $\operatorname{AS}_{0}^{\mathrm{N}}(\Phi ; \Psi)$ and $\mathrm{AS}_{0}^{\mathrm{N}}(\tilde{\Phi} ; \tilde{\Psi})$ are Bessel sequences in $L_{2}\left(\mathbb{R}^{D}\right)$. By Theorem 4.4, we see that $\left(\operatorname{FAS}_{0}^{\mathrm{N}}(\widehat{\Phi} ; \widehat{\Psi}), \operatorname{FAS}_{0}^{\mathrm{N}}(\widehat{\tilde{\Phi}} ; \widehat{\tilde{\Psi}})\right)$ is a pair of frequency-based dual framelets. Now it follows from [9, Theorem 9$]$ that $(\{\Phi ; \Psi\},\{\tilde{\Phi} ; \tilde{\Psi}\})$ is a dual $\mathrm{N}$-framelet in $L_{2}\left(\mathbb{R}^{D}\right)$.

By Theorem 2.3, we see that $\left(\left\{P a ; P b_{1}, \ldots, P b_{s}\right\},\left\{P \tilde{a} ; P \tilde{b}_{1}, \ldots, P \tilde{b}_{s}\right\}\right)_{P \Theta}$ is a dual M-framelet filter bank. Since $\phi$ and $\tilde{\phi}$ are vectors of compactly supported functions in $L_{2}\left(\mathbb{R}^{D}\right)$, we see that $P \phi$ and $P \tilde{\phi}$ are also vectors of compactly supported functions in $L_{2}\left(\mathbb{R}^{d}\right)$. Note that (5.7) implies

$$
\widehat{\widehat{P \phi}}(0)^{\top} \widehat{P \Theta}(0) \widehat{P \tilde{\phi}}(0)=1 \quad \text { and } \quad \widehat{P b_{\ell}}(0) \widehat{P \phi}(0)=\widehat{P \tilde{b}_{\ell}}(0) \widehat{P \tilde{\phi}}(0)=0, \quad \forall \ell=1, \ldots, s .
$$

By the same argument, we see that $(\{P \Phi ; P \Psi\},\{P \tilde{\Phi} ; P \tilde{\Psi}\})$ is a dual M-framelet in $L_{2}\left(\mathbb{R}^{d}\right)$.

Closely related to a nonhomogeneous affine system $\mathrm{AS}_{0}^{\mathrm{M}}(\Phi ; \Psi)$ in $L_{2}\left(\mathbb{R}^{d}\right)$, a homogeneous affine system is defined to be

$$
\operatorname{AS}^{\mathrm{M}}(\Psi):=\left\{\psi_{\mathrm{M}^{j} ; k}: j \in \mathbb{Z}, k \in \mathbb{Z}^{d}, \psi \in \Psi\right\} .
$$

We say that $\Psi$ is a homogeneous tight $\mathrm{M}$-framelet in $L_{2}\left(\mathbb{R}^{d}\right)$ if $\operatorname{AS}^{\mathrm{M}}(\Psi)$ is a normalized tight frame for $L_{2}\left(\mathbb{R}^{d}\right)$. We say that $(\Psi, \tilde{\Psi})$ is a homogenous dual $\mathrm{M}$-framelet in $L_{2}\left(\mathbb{R}^{d}\right)$ if $\left(\operatorname{AS}^{\mathrm{M}}(\Psi), \mathrm{AS}^{\mathrm{M}}(\tilde{\Psi})\right)$ is a pair of dual frames for $L_{2}\left(\mathbb{R}^{d}\right)$. The application of the projection method to homogeneous affine systems has been addressed in [11]. Since the paper [11] is not easily accessible, we recall two results here from [11] on applications of the projection method on homogeneous affine systems in $L_{2}\left(\mathbb{R}^{d}\right)$. For the convenience of the reader, we give a sketch of proof here. 
Theorem 5.6. ([11, Theorem 2.2]) Let $\mathrm{M}$ be a $d \times d$ expansive integer matrix and $\mathrm{N}$ be $a \mathrm{D} \times D$ expansive integer matrix. Let $P$ be a $d \times D$ integer matrix having rank d such that $(2.9)$ holds. If $\left\{\psi^{[1]}, \ldots, \psi^{[s]}\right\}$ is a homogeneous tight $\mathrm{N}$-framelet in $L_{2}\left(\mathbb{R}^{D}\right)$ and if

(i) all $\widehat{\psi^{[\ell]}}$ are continuous on $\mathbb{R}^{D}$ and $P \psi^{[\ell]} \in L_{2}\left(\mathbb{R}^{d}\right), \ell=1, \ldots, L$;

(ii) $\sum_{\ell=1}^{s} \sum_{j \in \mathbb{Z}}\left|\widehat{\psi^{[\ell]}}\left(\left(\mathrm{N}^{\top}\right)^{j} \cdot\right)\right|^{2}$ converges uniformly on any compact subset of $\mathbb{R}^{D} \backslash\{0\}$, then $\left\{P \psi^{[1]}, \ldots, P \psi^{[s]}\right\}$ is a homogeneous tight $\mathrm{M}$-framelet in $L_{2}\left(\mathbb{R}^{d}\right)$.

Proof. By our assumption in (2.9), we see that for any $\beta \in \mathbb{Z}^{d} \backslash\left[\mathrm{M}^{\top} \mathbb{Z}^{d}\right]$, we must have $P^{\top} \beta \in \mathbb{Z}^{D} \backslash\left[\mathrm{N}^{\top} \mathbb{Z}^{D}\right]$. For a finite subset $\Psi=\left\{\psi^{[1]}, \ldots, \psi^{[s]}\right\}$ of $L_{2}\left(\mathbb{R}^{D}\right)$, it is known in [21, Theorem 2.7], [22, Theorem 2.8] and [30] that $\Psi$ is a homogeneous tight $\mathrm{N}$-framelet in $L_{2}\left(\mathbb{R}^{D}\right)$ if and only if

$$
\begin{aligned}
& \left.\sum_{j \in \mathbb{Z}} \sum_{\ell=1}^{s} \widehat{\mid \psi^{[\ell]}}\left(\left(\mathbf{N}^{\top}\right)^{j} \zeta\right)\right|^{2}=1, \\
& \sum_{j=0}^{\infty} \sum_{\ell=1}^{s} \widehat{\widehat{\psi^{[\ell]}}\left(\left(\mathbf{N}^{\top}\right)^{j} \zeta\right)} \widehat{\psi^{[\ell]}}\left(\left(\mathbf{N}^{\top}\right)^{j}(\zeta+\gamma)\right)=0, \quad \forall \gamma \in \mathbb{Z}^{D} \backslash\left[\mathbf{N}^{\top} \mathbb{Z}^{D}\right]
\end{aligned}
$$

for almost every $\zeta \in \mathbb{R}^{D}$, with all series converging absolutely. By our assumption in item (ii), we conclude that the above two identities must hold for all $\zeta \in \mathbb{R}^{D} \backslash\{0\}$. Consequently, we have

$$
\sum_{j \in \mathbb{Z}} \sum_{\ell=1}^{s}\left|\widehat{P \psi^{[\ell]}}\left(\left(\mathrm{M}^{\top}\right)^{j} \xi\right)\right|^{2}=\left.\sum_{j \in \mathbb{Z}} \sum_{\ell=1}^{s} \widehat{\mid \psi^{[\ell]}}\left(\left(\mathrm{N}^{\top}\right)^{j} P^{\top} \xi\right)\right|^{2}=1
$$

and for every $\beta \in \mathbb{Z}^{d} \backslash\left[\mathrm{M}^{\top} \mathbb{Z}^{d}\right]$, we have $P^{\top} \beta \in \mathbb{Z}^{D} \backslash\left[\mathrm{N}^{\top} \mathbb{Z}^{D}\right]$ and

$$
\sum_{j=0}^{\infty} \sum_{\ell=1}^{s} \overline{\widehat{P \psi^{[\ell]}}\left(\left(\mathrm{M}^{\top}\right)^{j} \xi\right)} \widehat{P \psi^{[\ell]}}\left(\left(\mathrm{M}^{\boldsymbol{\top}}\right)^{j}(\xi+\beta)\right)=\sum_{j=0}^{\infty} \sum_{\ell=1}^{s} \widehat{\widehat{\psi^{[\ell]}}\left(\left(\mathbf{N}^{\top}\right)^{j} P^{\top} \xi\right)} \widehat{\psi^{[\ell]}}\left(\left(\mathbf{N}^{\top}\right)^{j}\left(P^{\boldsymbol{\top}} \xi+P^{\boldsymbol{\top}} \beta\right)\right)=0
$$

for all $\xi \in \mathbb{R}^{d} \backslash\{0\}$. By our assumption in item (i), we have $P \Psi \subseteq L_{2}\left(\mathbb{R}^{d}\right)$. By $[21,22,30]$, we conclude that $\left\{P \psi^{[1]}, \ldots, P \psi^{[s]}\right\}$ must be a homogeneous tight $\mathrm{M}$-framelet in $L_{2}\left(\mathbb{R}^{d}\right)$.

By a similar argument, we have

Theorem 5.7. ([11, Theorem 2.3]) Let $\mathrm{M}$ be a $d \times d$ expansive integer matrix and $\mathrm{N}$ be $a \mathrm{D} \times D$ expansive integer matrix. Let $P$ be a $d \times D$ integer matrix having rank $d$ such that (2.9) holds. Suppose that $\left(\left\{\psi^{[1]}, \ldots, \psi^{[s]}\right\},\left\{\tilde{\psi}^{[1]}, \ldots, \tilde{\psi}^{[s]}\right\}\right)$ is a homogeneous dual $\mathrm{N}$-framelet in $L_{2}\left(\mathbb{R}^{D}\right)$. If

(i) all $\widehat{\psi^{[\ell]}}, \widehat{\tilde{\psi}^{[\ell]}}$ are continuous on $\mathbb{R}^{D}$ and $P \psi^{[\ell]}, P \tilde{\psi}^{[\ell]} \in L_{2}\left(\mathbb{R}^{d}\right)$ for all $\ell=1, \ldots, s$;

(ii) $\sum_{j \in \mathbb{Z}} \sum_{\ell=1}^{s}\left[\left|\widehat{\psi^{[\ell]}}\left(\left(\mathbf{N}^{\top}\right)^{j} \cdot\right)\right|^{2}+\left|\widehat{\tilde{\psi}^{[\ell]}}\left(\left(\mathbf{N}^{\top}\right)^{j} \cdot\right)\right|^{2}\right]$ converges uniformly on any compact subset of $\mathbb{R}^{D} \backslash\{0\}$; and if in addition

(iii) $\operatorname{AS}^{\mathrm{M}}\left(\left\{P \psi^{[1]}, \ldots, P \psi^{[s]}\right\}\right)$ and $\mathrm{AS}^{\mathrm{M}}\left(\left\{P \tilde{\psi}^{[1]}, \ldots, P \tilde{\psi}^{[s]}\right\}\right)$ are Bessel sequences in $L_{2}\left(\mathbb{R}^{d}\right)$, then $\left(\left\{P \psi^{[1]}, \ldots, P \psi^{[s]}\right\},\left\{P \tilde{\psi}^{[1]}, \ldots, P \tilde{\psi}^{[s]}\right\}\right)$ is a homogeneous dual $\mathrm{M}$-framelet in $L_{2}\left(\mathbb{R}^{d}\right)$.

We complete this paper by some remarks on the periodization technique for obtaining periodic wavelets and framelets from wavelets and framelets on a Euclidean space $\mathbb{R}^{d}$. For $f \in L_{1}\left(\mathbb{R}^{d}\right)$, the periodization operator $\mathscr{P}: L_{1}\left(\mathbb{R}^{d}\right) \rightarrow L_{1}\left(\mathbb{T}^{d}\right)$ is defined to be

$$
\mathscr{P} f(x):=f^{p e r}(x):=\sum_{k \in \mathbb{Z}} f(x-k), \quad x \in \mathbb{R}^{d} .
$$


If $f \in L_{2}\left(\mathbb{R}^{d}\right)$ has compact support, then $\mathscr{P} f$ is a well-defined function in $L_{2}\left(\mathbb{T}^{d}\right)$.

Let $\mathrm{M}$ be a $d \times d$ expansive integer matrix. Suppose that $\{\Phi ; \Psi\}$ is an orthonormal $\mathrm{M}$-wavelet in $L_{2}\left(\mathbb{R}^{d}\right)$, that is, $\operatorname{AS}_{0}^{\mathrm{M}}(\Phi ; \Psi)$ is an orthonormal basis of $L_{2}\left(\mathbb{R}^{d}\right)$. If all the elements in $\Phi$ and $\Psi$ are compactly supported, then it is well known ([29]) that

$$
\{\mathscr{P}(\phi): \phi \in \Phi\} \cup \cup_{j=0}^{\infty}\left\{\mathscr{P}\left(\psi_{\mathrm{M}^{j} ; 0}\right)(\cdot-k): k \in \Gamma_{\mathrm{M}^{j}}, \psi \in \Psi\right\}
$$

is an orthonormal basis for $L_{2}\left(\mathbb{T}^{d}\right)$.

For $f \in L_{1}\left(\mathbb{R}^{d}\right)$, it is easy to directly check that $\mathscr{P} f \in L_{1}\left(\mathbb{T}^{d}\right)$ and for every $n \in \mathbb{Z}^{d}$,

$$
\widehat{\mathscr{P} f}(n):=\int_{[0,1]^{d}} \mathscr{P} f(x) e^{-i 2 \pi n \cdot x} d x=\int_{\mathbb{R}^{d}} f(x) e^{-i 2 \pi n \cdot x} d x=\widehat{f}(n),
$$

that is, $\widehat{\mathscr{P} f}=\left.\widehat{f}\right|_{\mathbb{Z}^{d}}$. In other words, in the frequency domain, the sequence $\mathscr{P} f$ is obtained from $\widehat{f}$ by restricting $\widehat{f}$ on the additive (discrete) subgroup $\mathbb{Z}^{d}$ of $\mathbb{R}^{d}$. Therefore, the periodization operator $\mathscr{P}$ in (5.9) can be regarded as a particular case of the projection method. Consequently, similar results in Sections 4 and 5 can be established for frequency-based periodic wavelets and framelets, for which we shall provide the detail elsewhere.

\section{References}

[1] C. de Boor, K. Höllig, S. Riemenschneider. Box splines. Series in Appl. Math. Sci. vol. 98. Springer-Verlag, New York, 1993.

[2] C. K. Chui. An introduction to wavelets. Academic Press, Inc., Boston, MA, 1992.

[3] C. K. Chui, W. J. He, J. Stöckler. Compactly supported tight and sibling frames with maximum vanishing moments. Appl. Comput. Harmon. Anal., 13 (2002), 224-262.

[4] I. Daubechies. Ten Lectures on Wavelets. CBMS-NSF Series, 61, SIAM, Philadelphia, 1992.

[5] I. Daubechies, B. Han. Pairs of dual wavelet frames from any two refinable functions. Constr. Approx., 20 (2004), 325-352.

[6] I. Daubechies, B. Han, A. Ron, Z. Shen. Framelets: MRA-based constructions of wavelet frames. Appl. Comput. Harmon. Anal., 14 (2003), 1-46.

[7] M. Ehler. On multivariate compactly supported bi-frames. J. Fourier Anal. Appl., 13 (2007), 511-532.

[8] B. Han. Properties of discrete framelet transforms. Math. Model. Nat. Phenom., 8 (2013), $18-47$.

[9] B. Han. Nonhomgeneous wavelet systems in high dimensions. Appl. Comput. Harmon. Anal., 32 (2012), $169-196$.

[10] B. Han. Pairs of frequency-based nonhomogeneous dual wavelet frames in the distribution space. Appl. Comput. Harmon. Anal., 29 (2010), 330-353.

[11] B. Han. Construction of wavelets and framelets by the projection method. Int. J. Appl. Math. Appl., 1 (2008), 1-40.

[12] B. Han. The projection method in wavelet analysis. in Splines and Wavelets: Athens 2005, G. Chen and M.J. Lai eds., (2006), 202-225.

[13] B. Han. Symmetric multivariate orthogonal refinable functions. Appl. Comput. Harmon. Anal., 17 (2004), $277-292$.

[14] B. Han. Computing the smoothness exponent of a symmetric multivariate refinable function. SIAM J. Matrix Anal. Appl., 24 (2003), 693-714.

[15] B. Han. Vector cascade algorithms and refinable function vectors in Sobolev spaces. J. Approx. Theory, 124 (2003), $44-88$.

[16] B. Han. Compactly supported tight wavelet frames and orthonormal wavelets of exponential decay with a general dilation matrix. J. Comput. Appl. Math., 155 (2003), 43-67.

[17] B. Han. Projectable multivariate refinable functions and biorthogonal wavelets. Appl. Comput. Harmon. Anal., 13 (2002), 89-102.

[18] B. Han. Symmetry property and construction of wavelets with a general dilation matrix. Linear Algebra Appl., 353, (2002), 207-225.

[19] B. Han. Approximation properties and construction of Hermite interpolants and biorthogonal multiwavelets. J. Approx. Theory, 110 (2001), 18-53.

[20] B. Han. Analysis and construction of optimal multivariate biorthogonal wavelets with compact support. SIAM J. Math. Anal., 31 (2000), 274-304.

[21] B. Han. On dual wavelet tight frames. Appl. Comput. Harmon. Anal., 4 (1997), 380-413.

[22] B. Han. Wavelets. M.Sc. thesis at the Institute of Mathematics, Chinese Academy of Sciences, China, 1994.

[23] B. Han, R. Q. Jia. Optimal $C^{2}$ two-dimensional interpolatory ternary subdivision schemes with two-ring stencils. Math. Comp., 75 (2006), 1287-1308. 
[24] B. Han, R. Q. Jia. Optimal interpolatory subdivision schemes in multidimensional spaces. SIAM J. Numer. Anal., 36, (1998), 105-124.

[25] B. Han, Q. Mo. Analysis of optimal bivariate refinable Hermite interpolants. Commun. Pure Appl. Anal., 6 (2007), 689-718.

[26] R. Q. Jia. Approximation properties of multivariate wavelets. Math. Comp., 67 (1998), 647-665.

[27] M. J. Lai, J. Stöckler. Construction of multivariate compactly supported tight wavelet frames. Appl. Comput. Harmon. Anal., 21 (2006), 324-348.

[28] J. Liao. New interpolatory subdivision schemes in computer graphics. M.Sc. thesis at the University of Alberta, Canada, 2004.

[29] Y. Meyer. Wavelets and operators. Cambridge University Press, Cambridge, 1992.

[30] A. Ron, Z. Shen. Affine systems in $L_{2}\left(\mathbb{R}^{d}\right)$ : the analysis of the analysis operator. J. Funct. Anal., 148 (1997), $408-447$. 